Portland State University

PDXScholar

7-16-2021

\title{
Using Quantitative Stereology on High Resolution SEM Images to Estimate Diatom Percentages
}

Ariadna Covarrubias Ornelas

Portland State University

Follow this and additional works at: https://pdxscholar.library.pdx.edu/open_access_etds

Part of the Civil Engineering Commons, and the Soil Science Commons Let us know how access to this document benefits you.

Recommended Citation

Covarrubias Ornelas, Ariadna, "Using Quantitative Stereology on High Resolution SEM Images to Estimate Diatom Percentages" (2021). Dissertations and Theses. Paper 5781.

https://doi.org/10.15760/etd.7652

This Thesis is brought to you for free and open access. It has been accepted for inclusion in Dissertations and Theses by an authorized administrator of PDXScholar. Please contact us if we can make this document more accessible: pdxscholar@pdx.edu. 


\title{
Using Quantitative Stereology on High Resolution SEM Images to Estimate Diatom Percentages
}

\author{
by
}

Ariadna Covarrubias Ornelas

A thesis submitted in partial fulfillment of the requirements for the degree of

Master of Science

Civil and Environmental Engineering

Thesis Committee:

Diane Moug, Chair

Arash Khosravifar

Richard Hugo

Portland State University

2021 
(C) 2021 Ariadna Covarrubias Ornelas 


\section{ABSTRACT}

Diatoms are single-celled organisms of various shapes and sizes typically found in aquatic environments. When diatoms die, the organic material decomposes, and the outer skeletons (i.e., frustules) settle and accumulate as sedimentary deposits. These soils, called diatomaceous soils, exhibit nontraditional behavior since the diatom particles are typically hollow skeletons composed of amorphous silica with intricately patterned and abrasive surfaces. Recent studies have shown that diatomaceous soils are challenging geomaterials since even a small percentage of diatom particles will notably affect engineering behavior. Furthermore, laboratory studies on diatomaceous soil mixtures have demonstrated that many engineering soil properties depend on the percentage of diatom particles. For example, liquid limit and plastic limit increase as the percentage of diatom particles increases. Although the percentage of diatom particles relates to geotechnical properties, there are currently few published correlations to quantify this relationship. This research has two objectives: (1) to develop a method to characterize diatom particle percentage for natural diatomaceous soils; and (2) to relate these percentages to physical properties. The soils for this project were sampled from southern and central Oregon in Pinecone Way, Chiloquin and Wickiup Junction, La Pine, and imaged using scanning electron microscopy 
(SEM) to obtain high resolution images. These images were then analyzed using quantitative stereology to estimate diatom particle percentages. The sample from the Pinecone Way field site had approximately $92 \%$ diatom content, while the sample from the Wickiup Junction field site had about $88 \%$ diatom content. These percentages are compared to measured soil properties to evaluate the relationship for these natural diatomaceous soils. The sample from the Pinecone Way field site had liquid and plastic limit values that agreed with previously published trends for high diatom content mixtures. Liquid limit and plastic limit data were not available for the Wikiup Junction site. 


\section{DEDICATION}

Para mi mami, mi papi, y mis hermanos:

Gracias por darme espacio para explorar.

To Nayeli and Dulce:

Thank you for being with me in this journey.

And

To LSAMP:

Thank you for becoming my family. 


\section{ACKNOWLEDGEMENTS}

The work presented in this thesis was made possible due to the support and the assistance of my family, my friends, my colleagues, my mentors, and my professors.

I would like to begin by acknowledging that my family had tremendous patience and love to give to me through this ongoing journey; that my friends understood and accepted that sometimes my academics came first and still we remain friends; that my colleagues took time from their own work to explain what I did not understand; that my mentors listened to what I was saying and saw what I did not; and that my professors challenged me to learn a different way of seeing things.

There is a long list of people and organizations that have helped and guided me throughout my academic journey to today, like Adelante Chicas, Dr. Josephine Pino, Pat Sanchez-Cottrill, Louis Stokes Alliance for Minority Participation (LSAMP) in STEM at Portland State University (PSU), Dr. Lorna Tran, Dr. Suzanne Estes, Dr. Todd Rosenstiel, Dr. Sarah Eppley, Dr. Luke Nave, and Dr. Kathryn Hofmeister just to name a few.

I would like to thank my committee members: Dr. Arash Khosravifar, Dr. Diane Moug, and Dr. Richard Hugo: Thank you for your support and guidance. 
I would like to specially acknowledge and thank Dr. Diane Moug for working with me, for listening to my Biochemistry approach, and for your patience to guide me toward engineering judgement. I appreciate the trust and freedom you gave me to venture into this discipline while working with you.

I would like to thank Greg Baty the manager for the Center for Electron Microscopy and Nanofabrication at PSU for training me to use the PSU's scanning electron microscope (SEM) and guiding me through troubleshooting when I ran into unexpected situations while at the SEM.

I would like to thank Jiayao Wang and Dr. Matt Evans from Oregon State University for working with me to understand the soil samples and the Matlab scripts, respectively.

Thanks to the PSU-LSAMP program and Dr. Joyce Pieretti for supporting students like Anika Walter who jumped into this project to help with image processing and fine-tuning the present approach.

I would also like to acknowledge the Oregon Department of Transportation for funding my training and use of the SEM.

Lastly, I would like to thank everyone in the Civil and Environmental Engineering department specially in the Geotechnical Engineering program who supported and helped me through my journey from my Biochemistry training to engineering judgement. 
TABLE OF CONTENTS

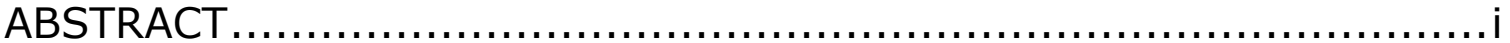

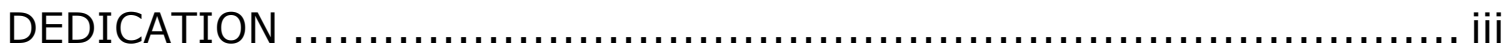

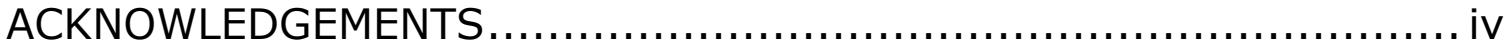

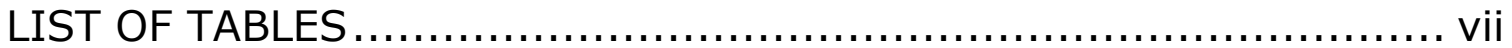

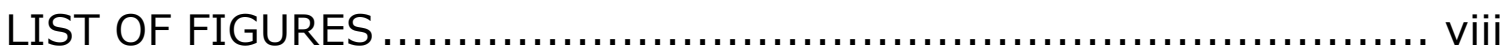

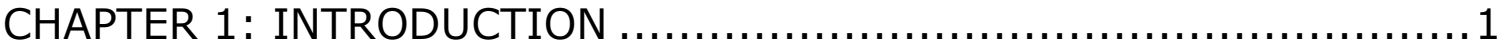

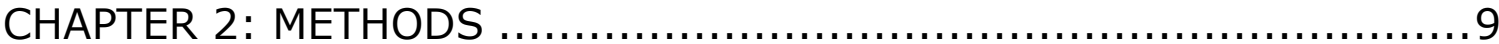

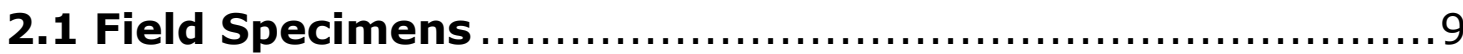

2.2 Specimen Preparation .......................................... 9

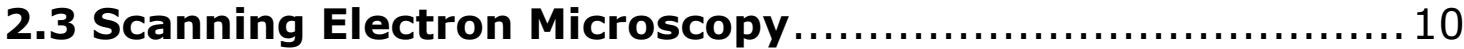

2.4 Image Processing ............................................. 12

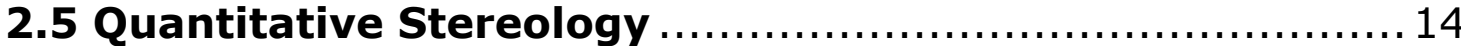

2.6 Calculations ................................................... 16

CHAPTER 3: DIATOM PERCENTAGES FROM SEM IMAGES ANALYSIS . 25

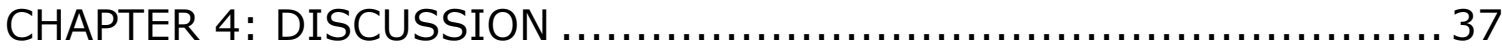

4.1 Number of Mosaic Images .................................. 37

4.2 Relationship Between Geotechnical Properties and Diatom Percentage .......................................................... 40

CHAPTER 5: CONCLUSIONS AND FUTURE RECOMMENDATIONS ...... 45

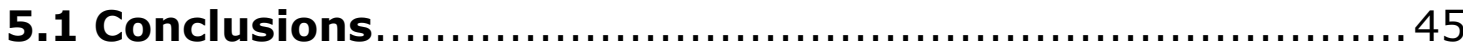

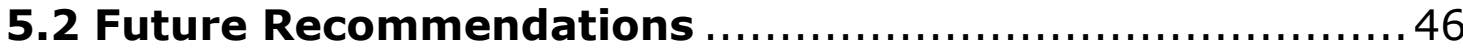

APPENDIX A: MOSAICS ............................................... 50

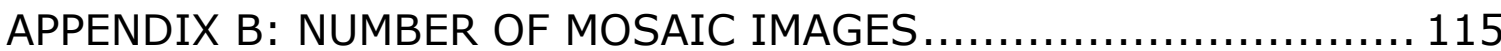

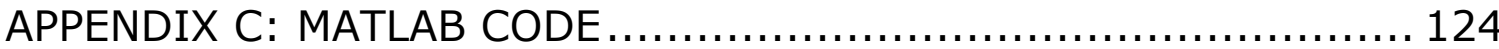




\section{LIST OF TABLES}

Table 3-1. Descriptive statistics for sample PC1N3 from Pinecone Way, Chiloquin broken down by specimen and mosaics................ 35 Table 3-2. Descriptive statistics for sample W1N10 from Wickiup Junction, La Pine broken down by specimen and mosaics. ............... 36 Table 4-1. Descriptive statistics for mosaic IV from specimen W1N1001 and mosaic II from specimen W1N10-02, both from Wickiup Junction. The mean values presented for the one image (1) are the raw estimated values of diatom percentages from each image. ....... 42 


\section{LIST OF FIGURES}

Figure 1-1. Living diatoms; (a) free-floating Amphora and (b) Gomphonema species attached to a substrate. Scale bar on the bottom right corner of each image measures $20 \mu \mathrm{m}$. Images from Kale and Karthick (2015). 4

Figure 1-2. Scanning electron micrographs of (a) a centric diatom, and (b) a pennate diatom with arrows indicating areolae - speciesspecific pores. Scale bar at the top right of each micrograph measures $2 \mu \mathrm{m}$. Image from Kale and Karthick (2015)............................4

Figure 1-3. Scanning electron micrographs of (a) Biddulphia reticulata (scale bar: $10 \mu \mathrm{m}$ ), (b) Diploneis sp. (scale bar: $10 \mathrm{um}$ ), (c) Eupodiscis radiatus (scale bar: $20 \mu \mathrm{m}$ ), and (d) Melosira varians (scale bar: $10 \mu \mathrm{m})$. Image from Bradbury (2004)........................... 5

Figure 1-4. Measured liquid limit (LL) and plastic limit (PL) in mixtures of Toyoura sand/Kaolin clay, diatomite/Kaolin clay, and diatomite/Singapore clay (from Shiwakoti et al. 2002) ..................6 Figure 1-5. Measured liquid limit (LL) and plastic limit (PL) in mixtures of crushed diatomaceous earth from Perma-Guard, Inc., and natural soils: (a) Hatch soil, and (b) Mesilla soil (from Al Shatnawi and Bandini 2018).

Figure 1-6. Compression index for mixtures of Kaolin clay/diatomite, Singapore clay/diatomite, and Kaolin clay/Toyoura sand mixtures (from Shiwakoti et al. 2002) ............................................

Figure 1-7. Sample collection sites in central and southern Oregon. ..8 Figure 2-1. Overall procedure. (a) Initial preparation of field specimens; (b) specimen preparation for scanning electron microscopy (SEM); (c) SEM imaging; (d) breakdown of specimens into quadrants for image capturing; (e) diagram of a four-by-four mosaic, 16 images total, captured per quadrant, from which nine (9) images in a threeby-three image mosaic were selected for cleaning; $(f)$ the selected 9 images were processed using quantitative stereology; $(\mathrm{g})$ results from quantitative stereology were used to estimate diatom percentages for each mosaic.

Figure 2-2. Versions of the SEM images captured from W1N10-01020 in tif format; (a) with information bar detailing SEM settings, and (b) without the information bar obscuring any part of the image. The information bar shows a scale bar of $3 \mu \mathrm{m}$, which applies for both images. Images were captured at a magnification of 3000. 
Figure 2-3.Images making up the mosaic from quadrant two, i.e., mosaic two (II) of specimen 01 of field sample W1N10 from Wickiup Junction, La Pine. Boxed are the selected nine images in a three-bythree image mosaic for quantitative stereology. Scale bar on top left image is of $80 \mu \mathrm{m}$ and applies to all images of the mosaic. Images were captured at a magnification of 3000 ....

Figure 2-4. Selected nine images cleaned to have diatom and nondiatom particles on a black background (i.e., features of the carbon tape were painted over). Scale bar on top left image is of $80 \mu \mathrm{m}$ and applies to all images of the mosaic. Images were captured at a magnification of 3000 .

Figure 2-5. Selected nine images cleaned to only have diatom particles on a black background. Scale bar on top left image is of 80 $\mu \mathrm{m}$ and applies to all images of the mosaic. Images were captured at a magnification of 3000 .

Figure 2-6. Histograms of (a) the original SEM image with the carbon tape, W1N10-01-020, and (b) the image with all soil particles on a black background, W1N10-01-020-01, from mosaic II from the Wickiup Junction field site. Marked by the " $x$ " is the cutoff value of grayscale applied, i.e., threshold value. The $\mathrm{x}$-axis represents the different grayscale values from 0 (black) to 255 (white)...............23

Figure 2-7. Processed images in binary after the threshold values were selected for each individual image. Scale bar on top left image is of $80 \mu \mathrm{m}$ and applies to all images of the mosaic. Images were captured at a magnification of 3000 .

Figure 3-1. Histograms of diatom particle proportions for samples:

(a) PC1N3 from Pinecone Way, Chiloquin and (d) W1N10 from Wickiup Junction, La Pine composed of the results of their specimen analysis; and diatom particle proportions from the sample specimens: (b) specimen 01, PC1N3-01, and (c) specimen 02, PC1N3-02, and (e) specimen 01, W1N10-01, and (f) specimen 02, W1N10-02........... 31 Figure 3-2. Data spread for sample PC1N3 from Pinecone Way, Chiloquin and its two (2) specimens, PC1N3-01 and PC1N3-02. ......32 Figure 3-3. Data spread for specimen PC1N3-01 from Pinecone Way, Chiloquin and its four (4) mosaics. ..................................... 32 Figure 3-4. Data spread for specimen PC1N3-02 from Pinecone Way, Chiloquin and its four (4) mosaics. ..................................... 33 Figure 3-5. Data spread for W1N10 from Wickiup Junction, La Pine and its two (2) specimens, W1N10-01 and W1N10-02................33 Figure 3-6. Data spread for W1N10-01 from Wickiup Junction, La Pine and its four (4) mosaics. ............................................... 34

Figure 3-7. Data spread for W1N10-02 from Wickiup Junction, La Pine and its four (4) mosaics.................................................. 34 
Figure 4-1. Diatom percentages estimated from images making up mosaic IV for the specimen W1N10-01 from Wickiup Junction..........41

Figure 4-2. Diatom percentages estimated from images making up mosaic II for the specimen W1N10-02 from Wickiup Junction. .........41 Figure 4-3. Liquid limit (LL) data published by Shiwakoti et al. (2002) and Al Shatnawi and Bandini (2018) adapted to include the LL of sample PC1N3 from the Pinecone Way, Chiloquin field site courtesy of Jiayao Wang (Ph.D. candidate, OSU).

Figure 4-4. Plastic limit (PL) data published by Shiwakoti et al. (2002) and Al Shatnawi and Bandini (2018) adapted to include the PL of sample PC1N3 from the Pinecone Way, Chiloquin field site courtesy of Jiayao Wang (Ph.D. candidate, OSU). 


\section{CHAPTER 1: INTRODUCTION}

Diatoms are eukaryotic unicellular organisms of various shapes and sizes found in aquatic environments, in particular lacustrine and marine environments, either as free-floating or attached to a substrate (Round et al., 1990; Kale and Karthick, 2015). They have porous cell walls, or outer skeletons, called frustules which allow for nutrient and waste exchange with their environment. These frustules are made up of transparent, opaline silica $\left(\mathrm{SiO}_{2} \cdot n \mathrm{H}_{2} \mathrm{O}\right)$. Figure 1-1 presents examples of free-floating and attached diatoms.

When diatoms die, the organic material decomposes, and the frustules settle and accumulate into sedimentary deposits. Figure 1-2 and Figure 1-3 show scanning electron microscope (SEM) micrographs of intact diatom particles. Figure 1-2, has a centric (i.e., has radial symmetry) and a pennate (i.e., has bilateral symmetry) diatom, while Figure 1-3 shows various examples of both centric and pennate diatoms. These soils, called diatomaceous soils, can be found in places such as Mexico City, Mexico, Osaka Bay, Japan, and California, United States (Díaz-Rodríguez and López-Molina, 2009) as well as in Peru, and Korea (Hardwood, 1999), and in Colombia (Caicedo et al., 2018).

Diatomaceous soils exhibit nontraditional behaviour which can be attributed to the high porosity (Hardwood, 1999) of the hollow amorphous silica skeletons which have intricate patterns and abrasive 
surfaces; and, if the skeletons are positioned "right", i.e., in a manner that reduces breakage, the frustules can remain more or less intact which can also contribute to a larger surface where water can sorb (Locat et al., 2003).

Studies have shown that diatomaceous soils are challenging geomaterials since even a small proportion of diatom particles will notably affect engineering behaviours. Laboratory studies, like those performed by Shiwakoti et al. (2002), on diatomite mixtures demonstrated an increase in liquid limit (LL) and plastic limit (PL) values with increasing diatom content (Figure 1-4 (b) and (c)); in contrast, when silt-size silica sand particles are added to kaolin clay, LL and PL decrease (Figure 1-4 (a)). Figure 1-5, presents the LL and PL of mixtures of two New Mexico natural soils, Hatch soil sampled from the Village of Hatch and Mesilla soil sampled from the Town of Mesilla, with diatomaceous earth which indicate that for both soils the $\mathrm{PL}$ and $\mathrm{LL}$ increase with increase diatomite content (Al Shatnawi and Bandini, 2018). Compressibility, another widely studied engineering behaviour, is presented in Figure 1-7, also from Shiwakoti et al. (2002), where it can be observed that the compression index increases as diatomite proportion increases (i.e., percentage of diatom particles). 
Although these studies indicate that knowing diatom percentage is important to estimating soil properties, currently there are few published correlations or methods to quantify the percentage of diatoms. This research has two objectives: (1) to develop a method to characterize diatom particle proportion for natural diatomaceous soils; and (2) to relate these percentages to physical properties. The soils for this project were sampled from southern and central Oregon in Pinecone Way, Chiloquin and Wickiup Junction, La Pine. Figure 1-7 is a map of Oregon with the sampling sites marked. Specimens from each sample at each site were imaged using scanning electron microscopy (SEM). These images were then analyzed using quantitative stereology to estimate diatom particle percentages. These proportions are compared to measured soil properties mainly LL and PL to evaluate the relationship for these natural diatomaceous soils. 


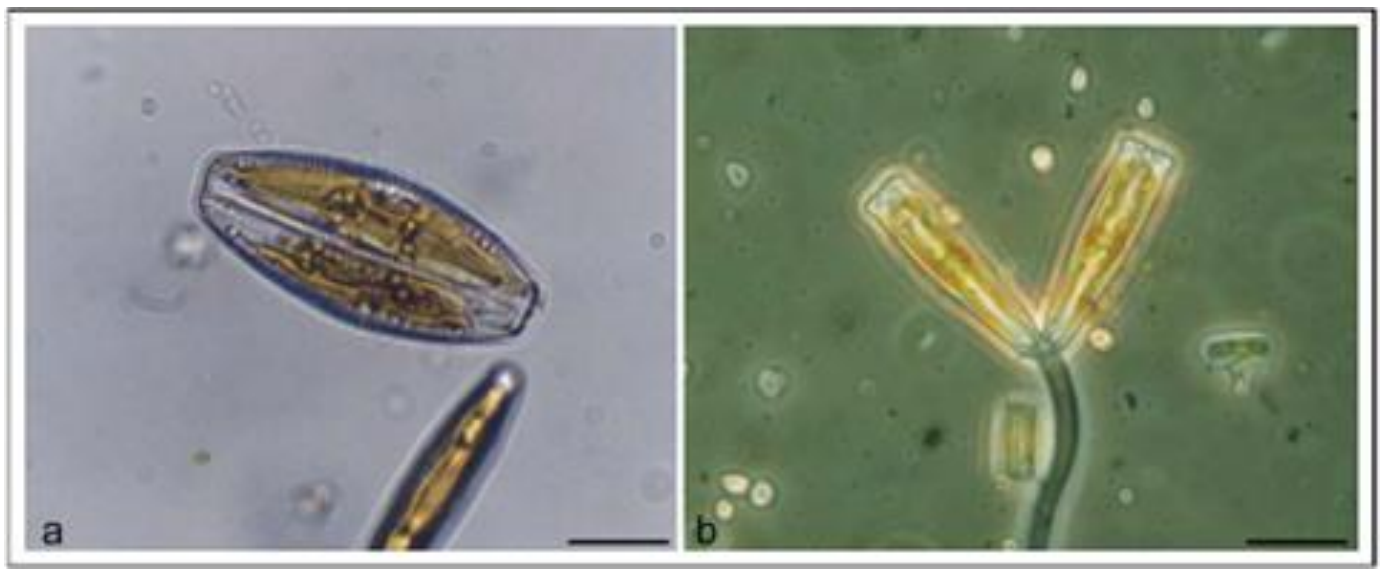

Figure 1-1. Living diatoms; (a) free-floating Amphora and (b) Gomphonema species attached to a substrate. Scale bar on the bottom right corner of each image measures $20 \mu \mathrm{m}$. Images from Kale and Karthick (2015).

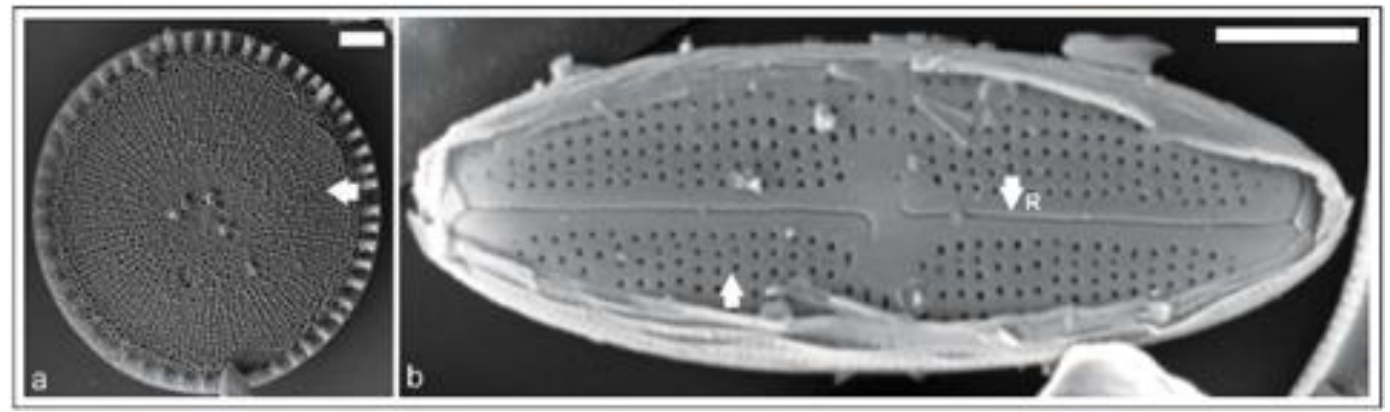

Figure 1-2. Scanning electron micrographs of (a) a centric diatom, and (b) a pennate diatom with arrows indicating areolae - speciesspecific pores. Scale bar at the top right of each micrograph measures $2 \mu \mathrm{m}$. Image from Kale and Karthick (2015). 
A

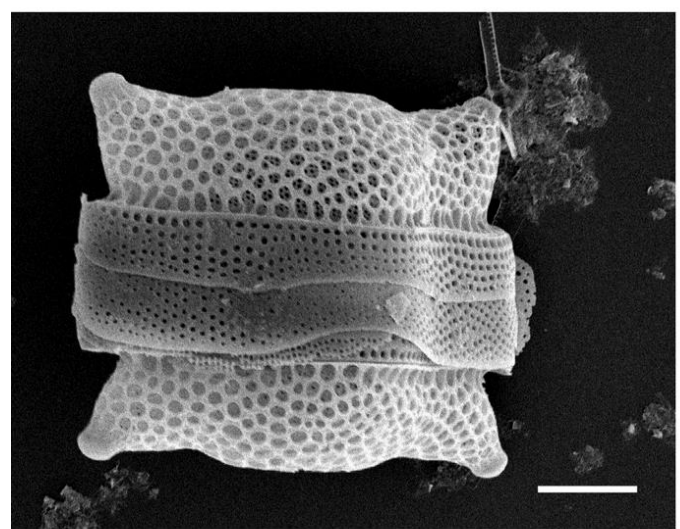

B

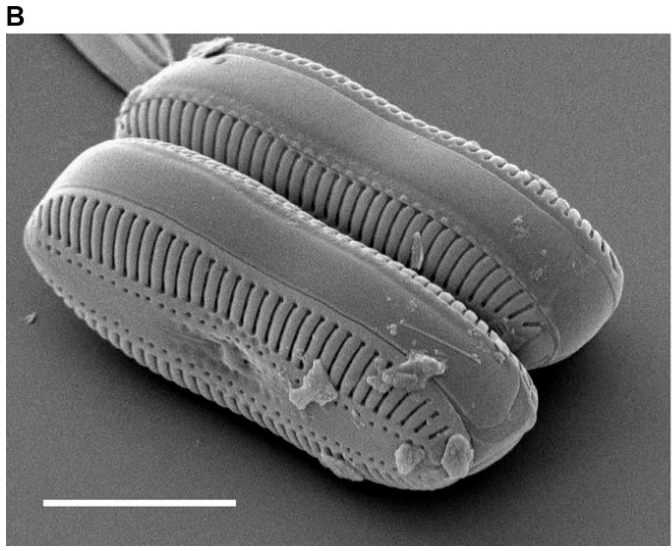

C

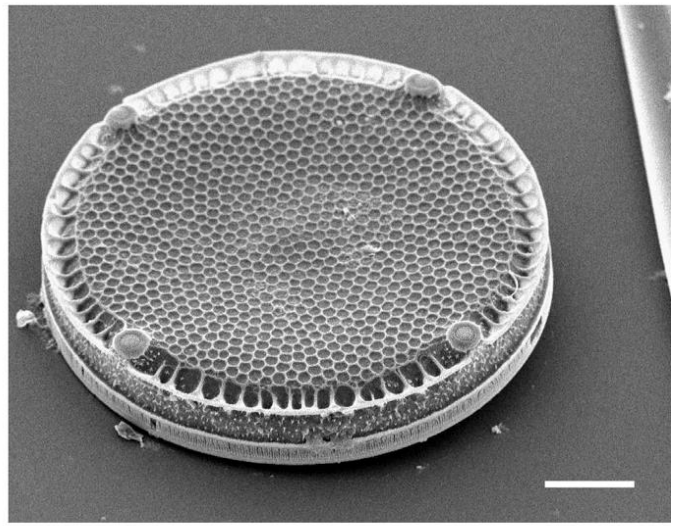

D

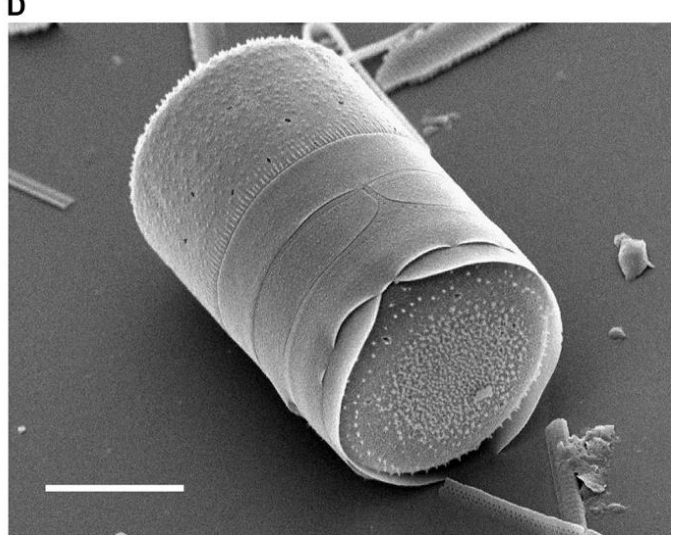

Figure 1-3. Scanning electron micrographs of (a) Biddulphia reticulata (scale bar: $10 \mu \mathrm{m}$ ), (b) Diploneis sp. (scale bar: $10 \mathrm{um}$ ), (c) Eupodiscis radiatus (scale bar: $20 \mu \mathrm{m}$ ), and (d) Melosira varians (scale bar: $10 \mu \mathrm{m})$. Image from Bradbury (2004). 


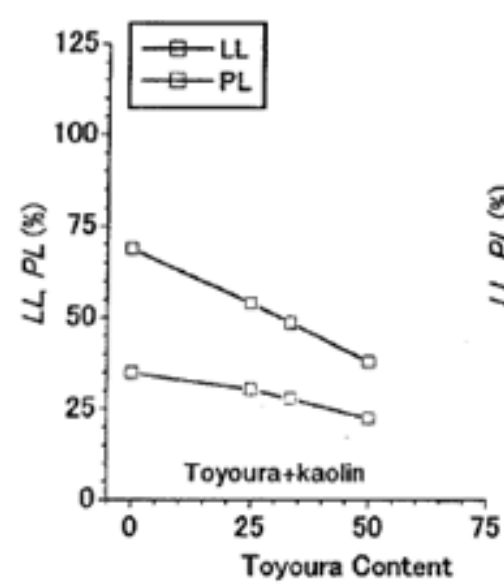

(a)

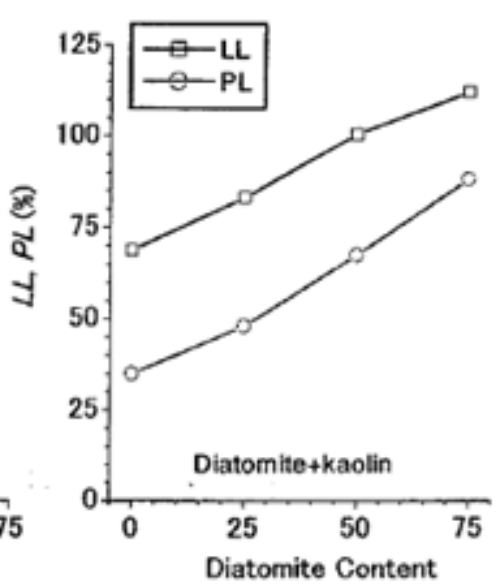

(b)

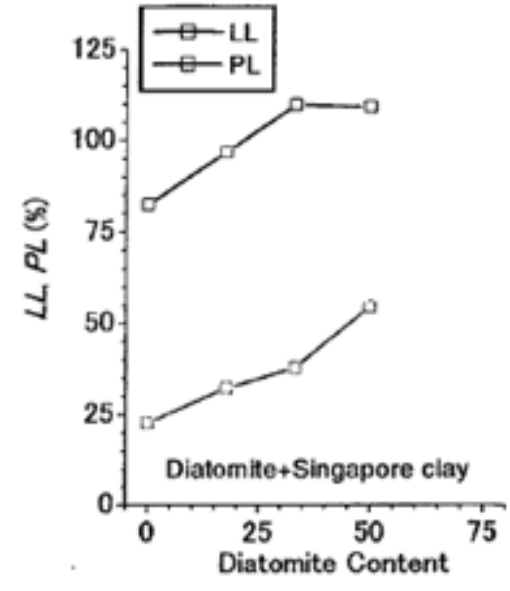

(c)

Figure 1-4. Measured liquid limit (LL) and plastic limit (PL) in mixtures of Toyoura sand/Kaolin clay, diatomite/Kaolin clay, and diatomite/Singapore clay (from Shiwakoti et al. 2002)
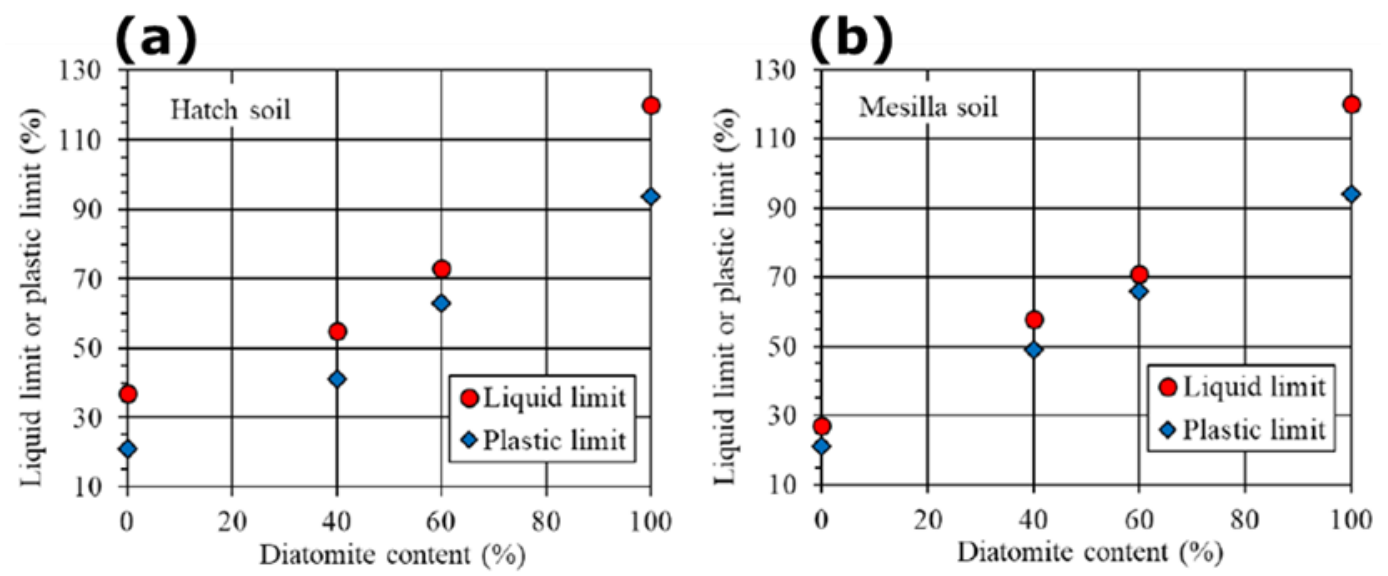

Figure 1-5. Measured liquid limit (LL) and plastic limit (PL) in mixtures of crushed diatomaceous earth from Perma-Guard, Inc., and natural soils: (a) Hatch soil, and (b) Mesilla soil (from Al Shatnawi and Bandini 2018). 


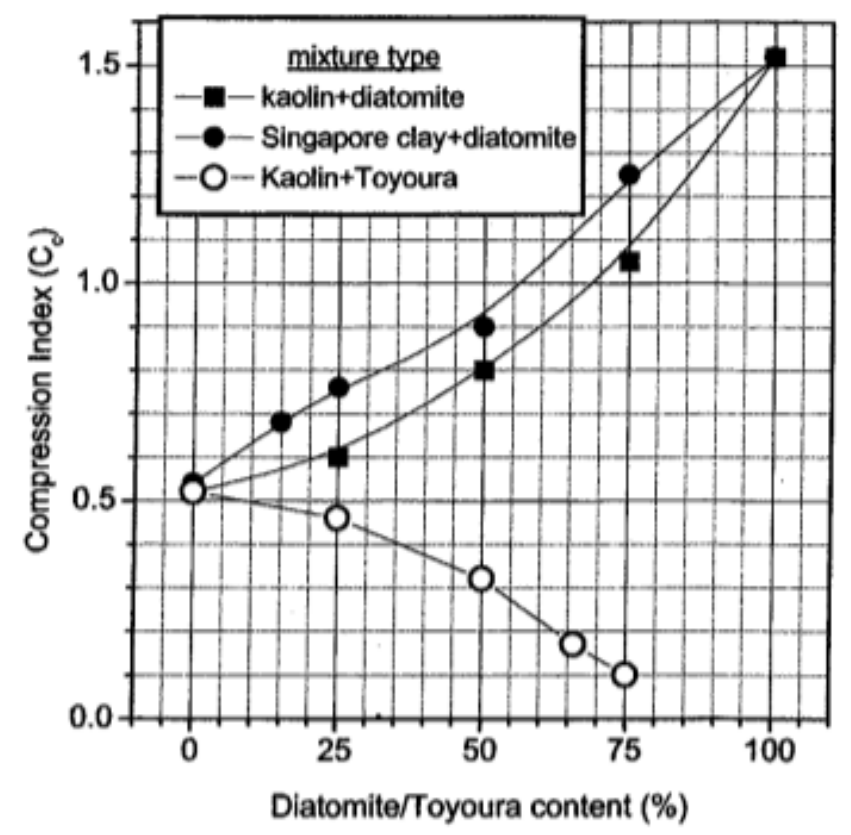

Figure 1-6. Compression index for mixtures of Kaolin clay/diatomite, Singapore clay/diatomite, and Kaolin clay/Toyoura sand mixtures (from Shiwakoti et al. 2002). 


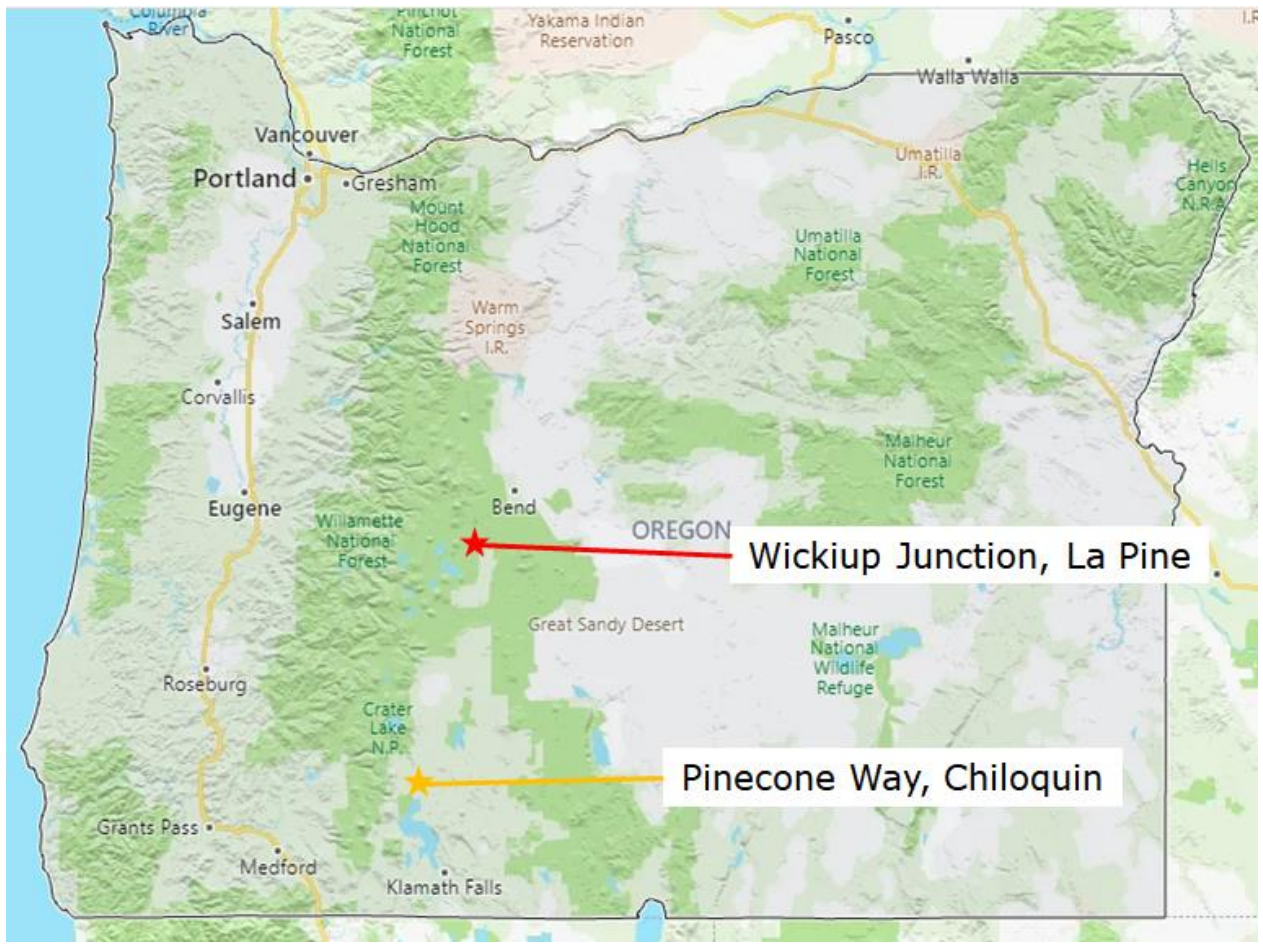

Figure 1-7. Sample collection sites in central and southern Oregon. 


\section{CHAPTER 2: METHODS}

\subsection{Field Specimens}

Samples were collected using a split-spoon test (SPT) sampler at sites in southern and central Oregon: Pinecone Way, Chiloquin and Wickiup Junction, La Pine. Samples were collected as part of ODOT project SPR-820.

Specimen preparation for SEM imaging aimed to separate particle clumps. Using a number 200 sieve the dry fines fraction of each specimen was brushed through the mesh to further separate the particles from one another. If clumps of particles were too large to pass through the mesh, they were mechanically crumbled to a size small enough to pass through. The samples selected for this study were $100 \%$ fine grained soils (i.e., all particles passed through the number 200 sieve).

An overview of the procedure for specimen preparation, imaging, and image processing is shown in Figure 2-1.

\subsection{Specimen Preparation}

To ensure uniformity for imaging, the specimens were mixed thoroughly in small plastic bags (Figure 2-1a). Then, a spoonful of the soil was scooped out and sprinkled on a piece of plastic wrap making sure there were no large clumps present. 
Next, a circular carbon tape (PELCO Tabs ${ }^{\mathrm{TM}}$ ) was placed onto an aluminum $1 / 8^{\prime \prime}$ pin or stub. Using $45^{\circ}$ angle tweezers the stub was turned upside down and positioned above the soil. The stub with the carbon tape was moved to lightly touch the soil to get a thin layer of soil onto the carbon tape (Figure 2-1b).

Once specimens were prepared, they were stored in a SEM storage box for pin mounts for transportation to the SEM microscopy lab. Specimens were prepared the same day they were to be imaged since the carbon tape changes in a way that affects the image background once exposed to air.

\subsection{Scanning Electron Microscopy}

A Zeiss Sigma VP FEG Scanning Electron Microscope with the SmartSEM system was used to capture images using the secondary electron detector (SE2), Figure 2-1c. Compressed nitrogen gas was used to create the high-pressure vacuum needed for the SEM.

Placing a gold coating on the specimens was found to create a charging effect that distorted the images; therefore, no gold coating was added to any specimen.

The SE2 was used with a voltage of $2.55 \mathrm{kV}$, an aperture of 30 $\mu \mathrm{m}$, and a collector bias of $100 \mathrm{~V}$. The SE2 provided the most surface detail compared to the other detectors available. 
The working distance varied depending on the specimen and the magnification of interest, i.e., 1000 and 3000, to yield the sharpest image. During the image capturing process the brightness and contrast were changed to yield the clearest image. To reduce the image noise line average was selected, and the scanning speed was reduced to a line scan of five (5) with an $\mathrm{N}$ of fourteen (14) to increase image quality. The images were stored at a resolution of 1024 by 768 pixels.

The stubs (i.e., specimens) were divided into four quadrants, in a manner similar to that illustrated in Figure 2-1d, where the arrow keys were used to capture a mosaic of four-by-four (4x4) images for a total of sixteen (16) images per quadrant, Figure 2-1e. This method of imaging was devised in order to maximize scheduled SEM sessions and organize the image capturing procedure. From a $4 \times 4$ mosaic, nine (9) images forming a three-by-three $(3 \times 3)$ mosaic were chosen for further analysis (Figure 2-3). The $3 \times 3$ mosaic analysis was advantageous since it took notably less time than a $4 \times 4$ mosaic, without compromising analysis quality (see Discussion section). The numbering of the quadrants was changed from specimen to specimen and within each quadrant to maintain a sense of "randomness" to the image capturing procedure, an image at a magnification of 1000 was captured to orient and select the location of each mosaic. However, 
the images for the mosaics were captured at a magnification of 3000 to increase the amount of detail visible in each image. The specific location of the mosaic within each quadrant was randomly chosen.

\subsection{Image Processing}

The images captured by the SEM were processed so that they could be analyzed using quantitative stereology which required binary image format. The images captured in the SEM were saved in tif format, one with a bar covering the bottom section of the image with information about the SEM settings used (Figure 2-2a) and another without said bar (i.e., plain images, Figure 2-2b). For this procedure, the plain images were used to make use of all information captured, and thus they were converted to bmp format using the ImageJ program. ImageJ was also used to process the images to separate diatom and non-diatom particles.

The images were prepared for quantitative stereology analysis using a process referred to as cleaning. First, images were inverted to have a white background to manually color over the carbon tape using the white brush feature in ImageJ. Then, the images were inverted again to the original color scheme, i.e., all soil particles on a black background, and saved (Figure 2-4). Next, these images were inverted again to manually color any non-diatom particles, e.g., particles that 
did not seem to have any pores visible, and particles smaller than 1 $\mu \mathrm{m}$ (Figure 2-5). The $1 \mu \mathrm{m}$ cut-off guideline was introduced due to the difficulty to differentiate between diatom and non-diatom particles if these particles had lengths smaller than $1 \mu \mathrm{m}$ as well as to expedite the process and reduce potential personal bias. Furthermore, clay particles are considered to be smaller than $2 \mu \mathrm{m}$ by AASHTO particle size classification, which is captured by the $1 \mu \mathrm{m}$ threshold. Lastly, these images, i.e., only diatom particles on a black background, were saved in bmp format.

The manual cleaning method is limited by what can be observed on the image which can lead to an under-estimation or an overestimation of diatom particles as well as personal bias. Underestimation can be due to cleaning out diatom particles smaller than 1 $\mu \mathrm{m}$, diatom particles that do not have obvious visible pores, and diatom particles that were partially obscured by non-diatom particles. Overestimation can occur due to counting non-diatom particles stuck to the edges of diatom particles. Depending on the person doing the manual cleaning there can be bias on whether a particle is diatom or non-diatom therefore influencing the particles that get cleaned out of an image. 


\subsection{Quantitative Stereology}

Quantitative stereology methods were used to estimate the volumetric proportion of diatom particles relative to all particles in an SEM image. Quantitative stereology makes use of two-dimensional (2D) images to infer measurements of interest of three-dimensional (3D) structures (Underwood, 1970). One of the relationships of quantitative stereology is that the $2 \mathrm{D}$ representation of an image represents the conditions in 3D. Since most of the diatom particles in the SEM images were broken and lie flat, a reasonable approximation of the physical volume ratio can be inferred from the image area ratio. Therefore, this analysis assumes that the proportion of diatom particles relative to all soil particles in the 2D SEM images is the same as the overall volumetric proportion of diatom particles in all the soil particles. The stereology approach and processing Matlab scripts were adapted from Evans and Frost (2010) which take an image convert it to binary and calculate the void ratio of the image based on pixel count.

The steps performed using the Matlab code included in Appendix C are:

- Access the images by setting up working directories;

- Indicate the format of the images to be analyzed and the format in which output images will be saved; 
- Indicate the image of interest;

- Obtain the grayscale histogram of the image of interest to place a cut-off value to designate a pixel as a zero ( 0 , black) or a one (1, white), where black is the background (i.e., void space) and white represents particles or part of particles of interest (i.e., non-diatom and diatom particles);

- Figure 2-6 present the resulting histograms for the original image W1N10-01-020 (from Figure 2-2) and the image W1N10-01-020 with all soil particles on a black background and the location of the threshold value applied.

- From these histograms it can be observed that there is a large amount of noise removed, i.e., there are less counts per grayscale value, when the features of the carbon tape are painted over to yield the image with all particles on $\mathrm{s}$ black background.

- Use the threshold value to convert the image in bmp format to binary, Figure 2-7 presents all the images from W1N10-01 mosaic II after the threshold values were applied to each image accordingly;

- Count the number of white pixels versus the number of black pixels; and 
- Calculate the void ratio, $e$, based on black to white pixel ratio from an image with all particles and the same image with only diatom particles.

\subsection{Calculations}

The definition of void ratio, $e$, in terms of volume was expressed in terms of image area, i.e., ratio of pixels of particles and nonparticles, and used to estimate diatom percentage in each image, Figure $2-1 \mathrm{~g}$.

The calculations began with,

$e=\frac{V_{V}}{V_{S}}=\frac{A_{V}}{A_{S}}$

Where, $V_{V}$ is the volume of voids, $V_{S}$ is the volume of solids, $A_{V}$ is the area of voids, and $A_{S}$ is the area of solids. Note that this is the void ratio represented in the image and will be used to estimate diatom particle proportion in the soil. The void ratio does not reflect a realistic void ratio for the soil's in-situ conditions.

Next, the definition of $e$ for each set of processed images image with all particles and image with only diatom particles - was defined using equation (1) as equation (2) and (3) below.

$e_{\text {all particles }}=\frac{A_{V}}{A_{S}}=\frac{A_{\text {total }}-A_{\text {all }} \text { particles }}{A_{\text {all }} \text { particles }}$ 
Where, $e_{\text {all particles }}$ is the void ratio of the image where all particles are present, $A_{\text {total }}$ is the total area, and $A_{\text {all particles }}$ is the area of all particles within the image.

$e_{\text {diatoms }}=\frac{A_{\text {total }}-A_{\text {diatoms }}}{A_{\text {diatoms }}}$

Where, $e_{\text {diatoms }}$ is the void ratio of the image with only diatom particles, and $A_{\text {diatoms }}$ is the area of diatom particles within the image.

Equations (2) and (3) were re-arranged to isolate $A_{\text {all particles }}$ and $A_{\text {diatoms }}$, respectively, in the following equations,

$A_{\text {all particles }}=\frac{A_{\text {total }}}{e_{\text {all particles }}+1}$

$A_{\text {diatoms }}=\frac{A_{\text {total }}}{e_{\text {diatoms }}+1}$

The area of diatoms, $A_{\text {diatoms }}$, equation (4) is then divided by the area of all particles, $A_{\text {all particles, }}$ equation (5) to estimate the diatom proportion within a set of images,

DiatomProportion $=\frac{A_{\text {diatoms }}}{A_{\text {all particles }}}=\frac{e_{\text {all particles }}+1}{e_{\text {diatoms }}+1}$

Lastly, the resulting proportions were multiplied by $100 \%$ to get the corresponding diatom percentages per image. 


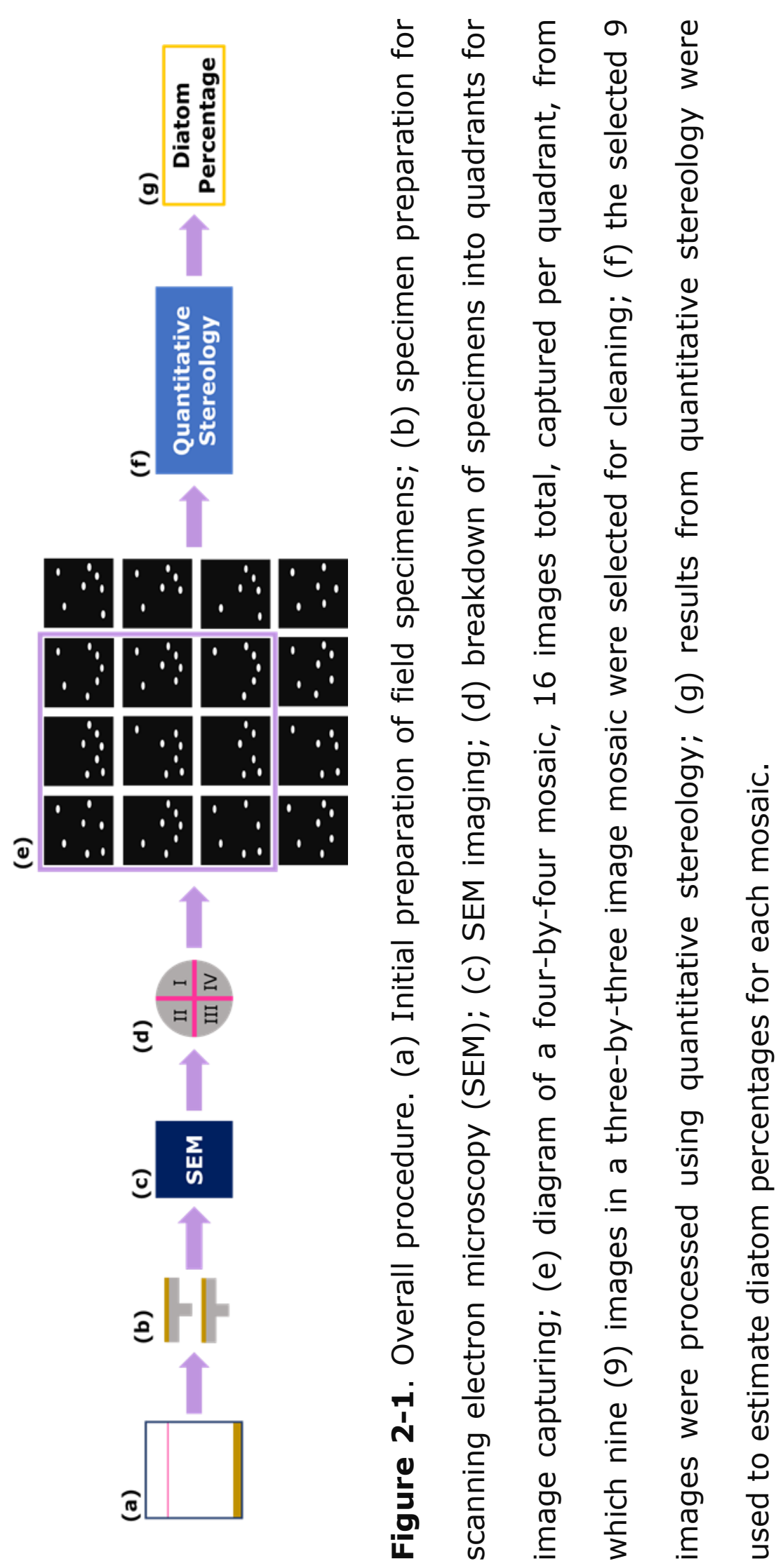




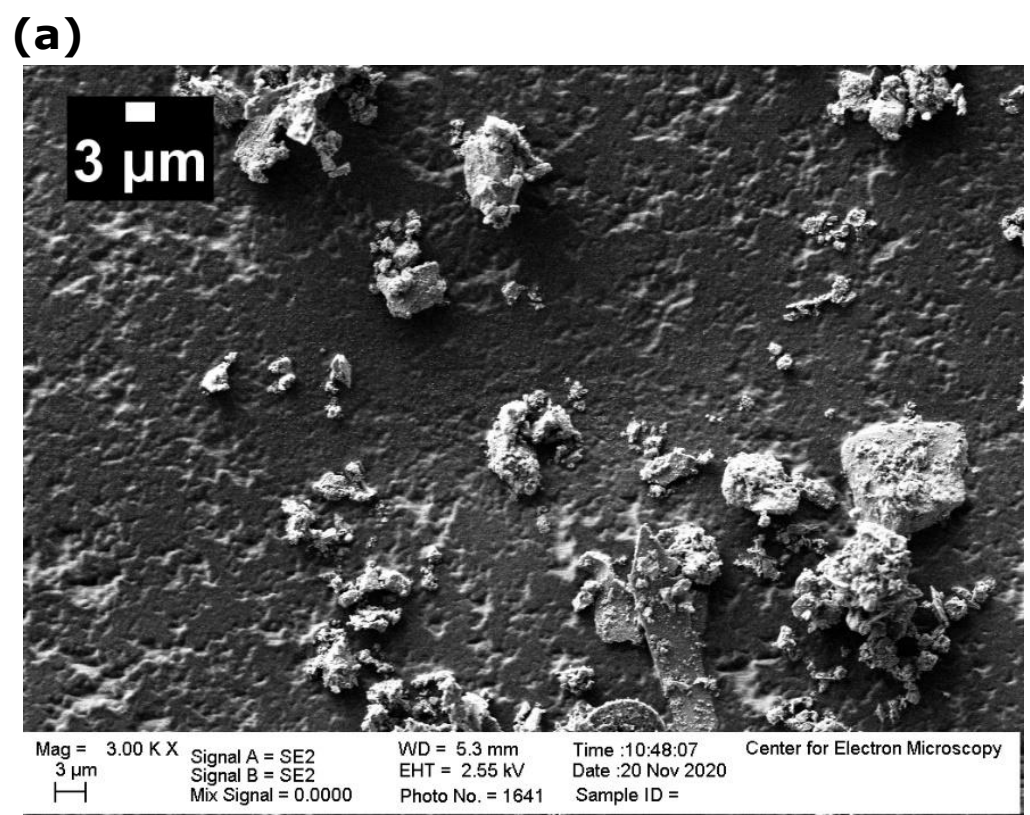

(b)

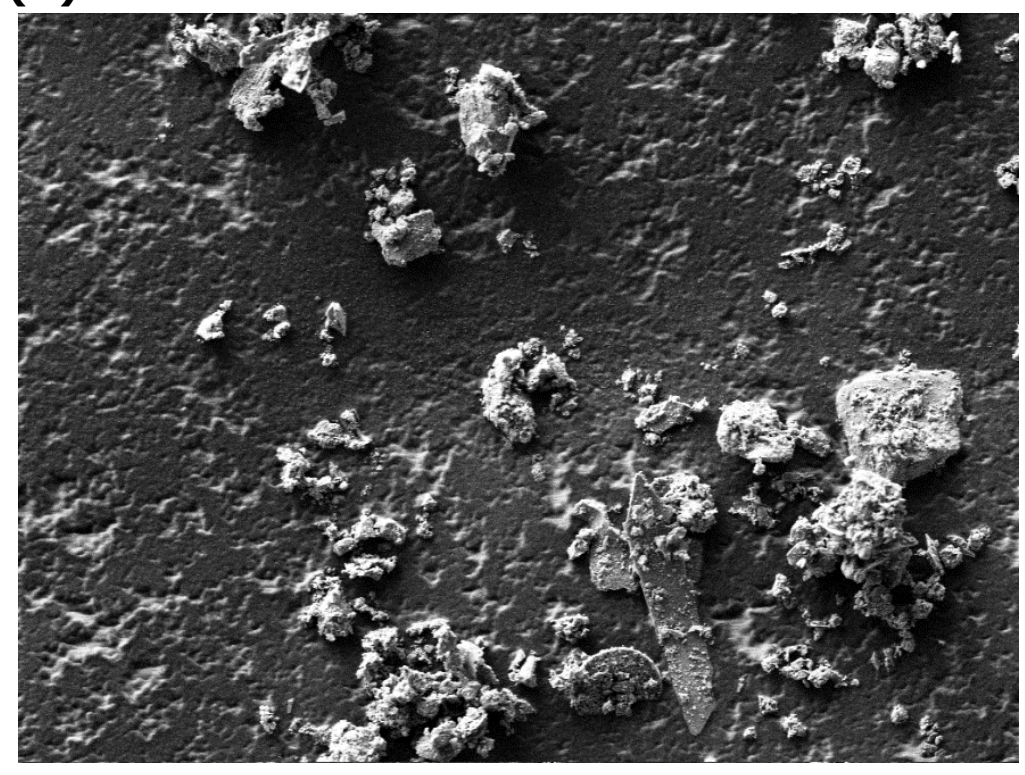

Figure 2-2. Versions of the SEM images captured from W1N10-01020 in tif format; (a) with information bar detailing SEM settings, and (b) without the information bar obscuring any part of the image. The information bar shows a scale bar of $3 \mu \mathrm{m}$, which applies for both images. Images were captured at a magnification of 3000 . 

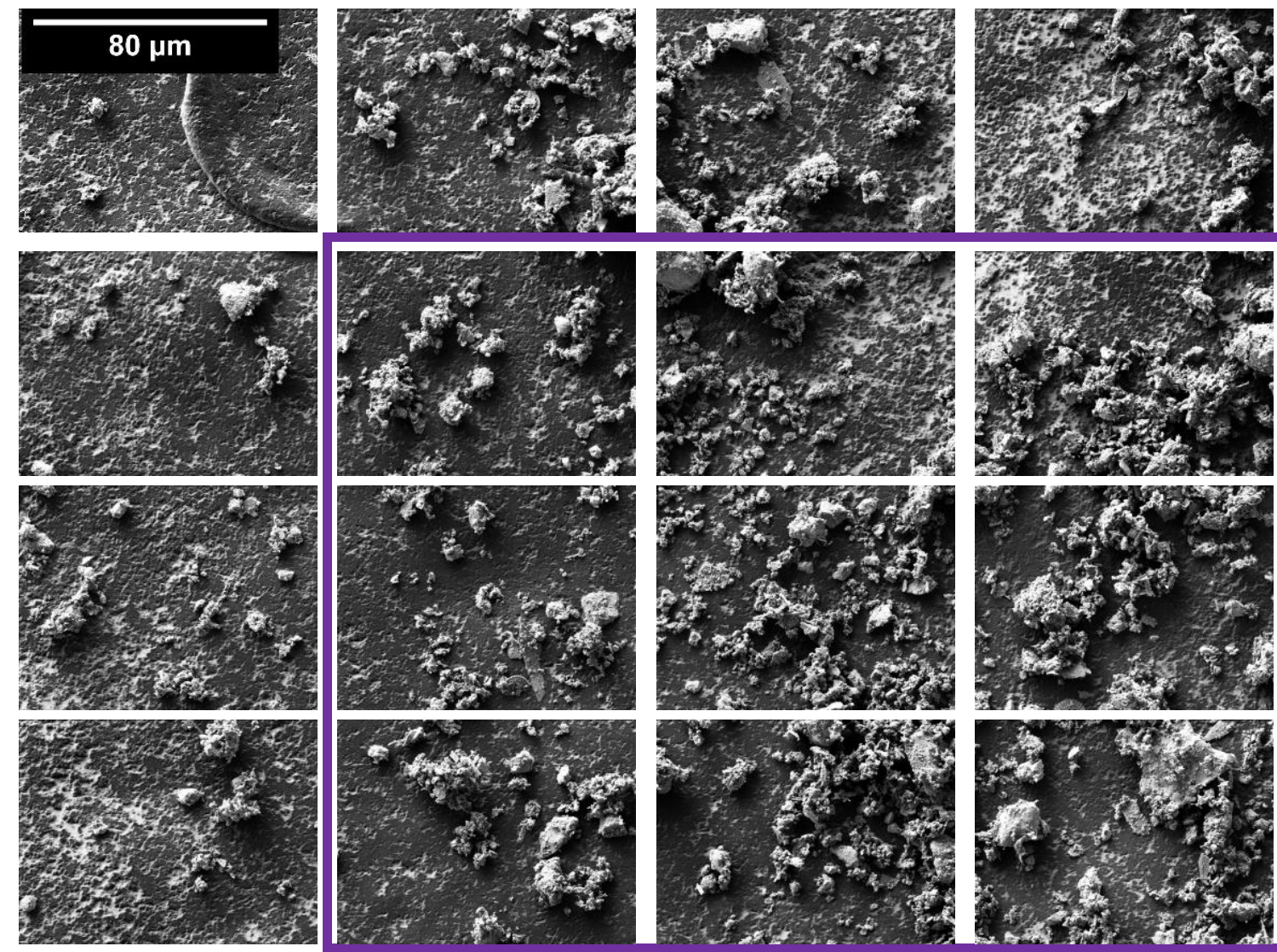

Figure 2-3.Images making up the mosaic from quadrant two, i.e., mosaic two (II) of specimen 01 of field sample W1N10 from Wickiup Junction, La Pine. Boxed are the selected nine images in a three-bythree image mosaic for quantitative stereology. Scale bar on top left image is of $80 \mu \mathrm{m}$ and applies to all images of the mosaic. Images were captured at a magnification of 3000 . 


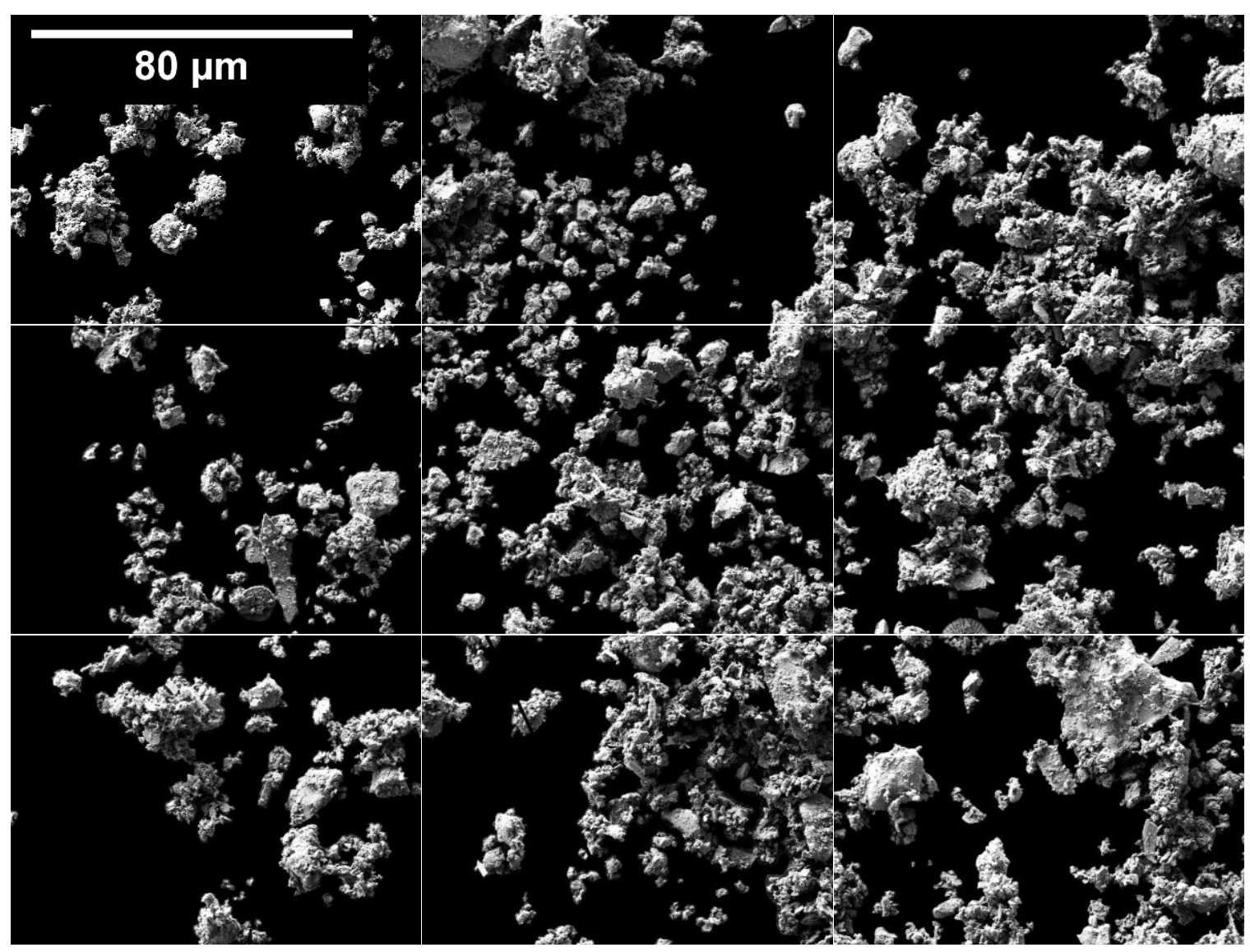

Figure 2-4. Selected nine images cleaned to have diatom and nondiatom particles on a black background (i.e., features of the carbon tape were painted over). Scale bar on top left image is of $80 \mu \mathrm{m}$ and applies to all images of the mosaic. Images were captured at a magnification of 3000 . 


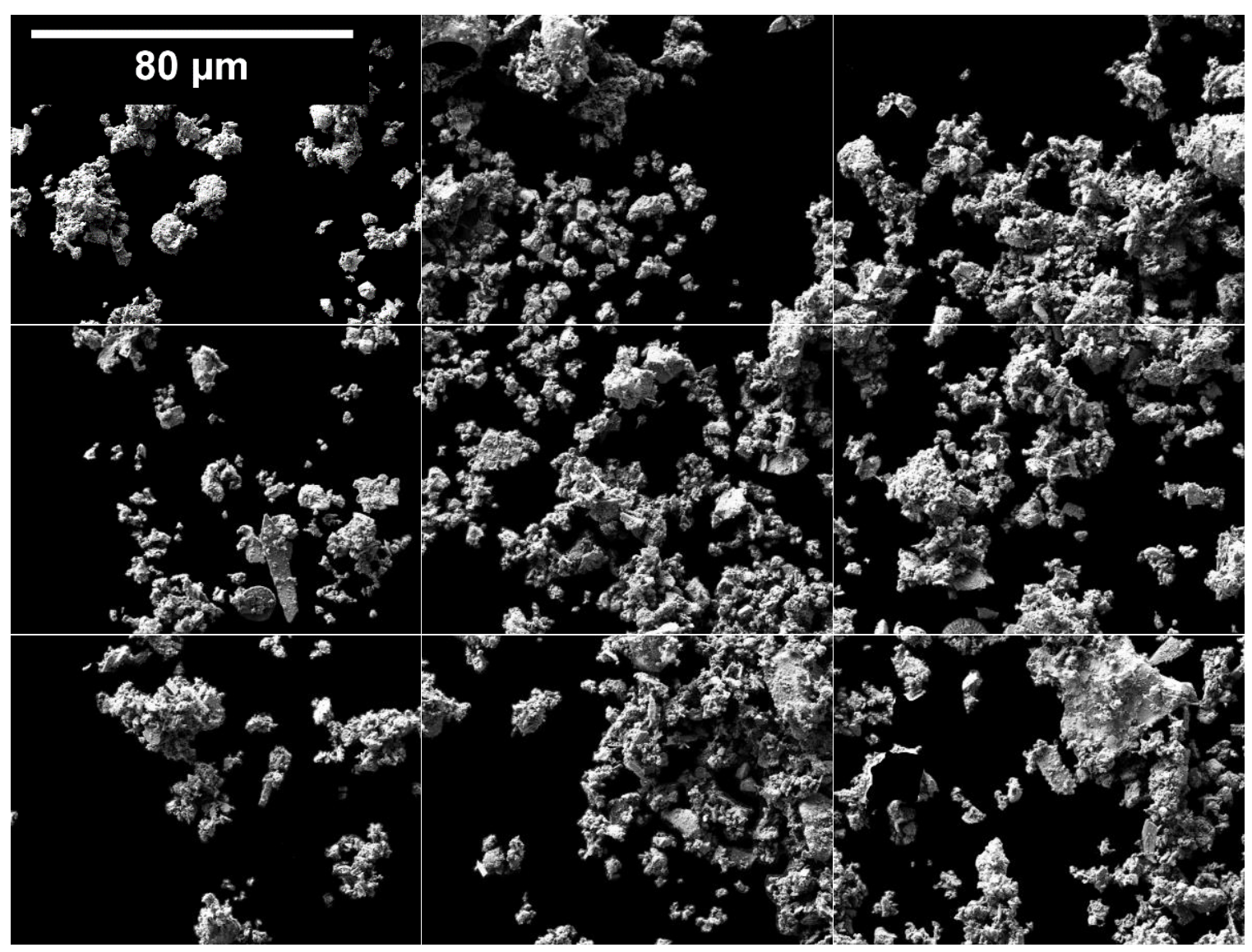

Figure 2-5. Selected nine images cleaned to only have diatom particles on a black background. Scale bar on top left image is of 80 $\mu \mathrm{m}$ and applies to all images of the mosaic. Images were captured at a magnification of 3000 . 

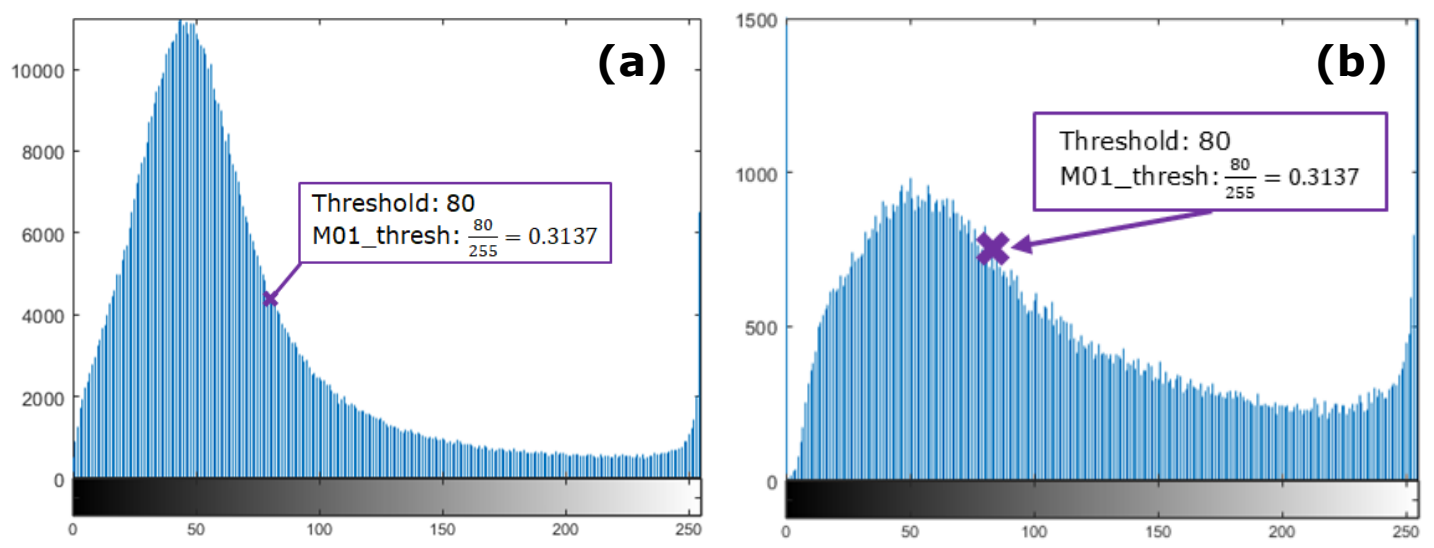

Figure 2-6. Histograms of (a) the original SEM image with the carbon tape, W1N10-01-020, and (b) the image with all soil particles on a black background, W1N10-01-020-01, from mosaic II from the Wickiup Junction field site. Marked by the " $x$ " is the cutoff value of grayscale applied, i.e., threshold value. The $\mathrm{x}$-axis represents the different grayscale values from 0 (black) to 255 (white). 


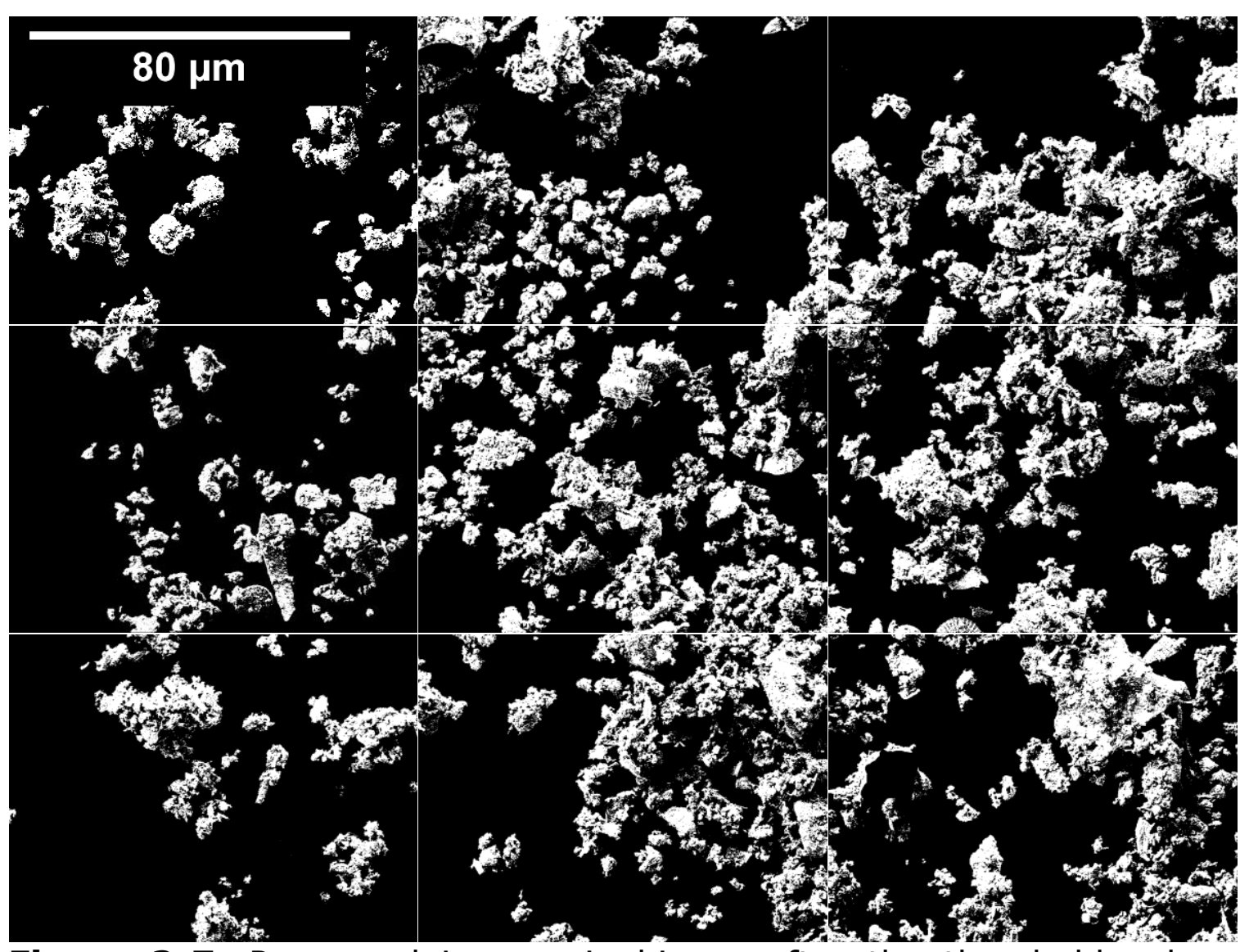

Figure 2-7. Processed images in binary after the threshold values

were selected for each individual image. Scale bar on top left image is of $80 \mu \mathrm{m}$ and applies to all images of the mosaic. Images were captured at a magnification of 3000 . 


\section{CHAPTER 3: DIATOM PERCENTAGES FROM SEM IMAGES ANALYSIS}

Two samples of diatomaceous soil were analyzed for this study. One from the Pinecone Way field site (PC1N3), and one from the Wickiup Junction field site (W1N10). From each sample, two (2) specimens were prepared for SEM imaging and subsequent analysis of diatom percentages. From each specimen four (4), three-by-three (3x3) image mosaics were analyzed for a total of eight (8) mosaics per sample (i.e., 72 images). This section analyzes the image mosaics to evaluate (i) the proportion of diatom particles to non-diatom particles, and (ii) how the data are distributed to evaluated variability of the analysis. Descriptive statistics were used to analyze the data.

Histograms of the samples and their respective specimens were constructed to explore the distribution of the data. Figure 3-1 presents six (6) histograms from analysis of the four (4) specimens and the composite result of the two (2) specimens from each sample. Since there are only nine (9) data points per mosaic, creating histograms for each would not provide additional insight to the distribution of the data. The histograms presenting the data for PC1N3 from the Pinecone Way field site and its two (2) specimens had bin sizes of 5\% (Figure 3-1 (a) to (c)); while the histograms presenting the data for W1N10 from the Wickiup Junction field site and its two (2) specimens had bin sizes of $10 \%$. Generally, the data distribution is tight and mostly 
presents values between 80 to $100 \%$ diatom content. The data appears skewed to the left; however, it must be noted that there is a natural limit at $100 \%$ since the specimens cannot have more than $100 \%$ diatom content.

Box-and-whisker plots were also used to investigate the spread of the data, as shown in Figures 3-2 to 3-7. Figure 3-2 presents the spread of the PC1N3 sample from the Pinecone Way field site and its two (2) specimens, PC1N3-01 and PC1N3-02. Figure 3-3 presents the spread of PC1N3-01 specimen along with its four (4) mosaics labeled mosaic I, mosaic II, mosaic III, and mosaic IV. In the same manner Figure 3-4 presents the spread of PC1N3-02 specimen and its corresponding mosaics. The means for both specimens agree with each other, PC1N3-01: $92.5 \%$ and PC1N3-02: $91.7 \%$, and they are adequately represented in the sample mean, PC1N3: $92.1 \%$. Specimen PC1N3-02 does have a larger spread than PC1N3-01, however this difference was considered reasonable for the analysis. The analysis from image mosaics for PC1N3-01 in Figure 3-3 show a small spread overall. Figure 3-3 shows two (2) outliers which are 1.5 times larger than the length of the interquartile range (IQR), one in mosaic III at $100 \%$ and the other in mosaic IV at about $73 \%$. The outlier in mosaic IV accounts for the outlier in specimen PC1N3-01. In Figure 3-4, PC1N3-02 mosaics I, II, and III have a relatively small 
spread in the data with mosaic III having the smallest spread with no outliers. Mosaics II and IV have one and two outliers, respectively. Together these three outliers account for the outliers present in PC1N3-02. It is also worth noting that mosaic IV drives the mean for the specimen down. Although these outliers are important the fact that sample PC1N3 has a relatively large data set means that they do not hold significant influence on the sample mean or median.

Figure 3-5 presents the spread of the W1N10 sample from the Wickiup Junction field site, against its two (2) specimens, W1N10-01 and W1N10-02. Figure 3-6 presents the data spread for specimen W1N10-01 along with the spread of its mosaics while Figure 3-7 presents specimen $\mathrm{W} 1 \mathrm{~N} 10-02$ and its mosaics.

From Figure 3-5, it can be observed that the spread of W1N10 is relatively large with three (3) outliers. All of which are from images within specimen W1N10-01 which also drives the large spread of the data. In Figure 3-6, W1N10-01 mosaics I, II, and IV have a relatively small spread in contrast with mosaic III. Mosaics I and II have outliers that do not show up as outliers in the specimen spread due to the smaller values and larger spread of mosaic III. Mosaic III also has an outlier that does show up in the box-and-whiskers plot for its specimen, along with the value represented by the bottom whisker. Also, the mean from mosaic III, $68.7 \%$, drives the specimen mean 
down to be $87.7 \%$. Specimen W1N10-02, Figure $3-7$, has a relatively smaller spread with no outliers which tightens the data spread for the sample allowing the outlier from W1N10-01 mosaic I to appear as an outlier in the box-and-whisker plot for the sample. Mosaic I has the largest spread when compared to the other mosaics, and mosaic IV has the smallest spread with one outlier. This outlier does not show up in the specimen spread.

To continue the analysis of the diatom percentages descriptive statistics such as the mean, standard error, standard deviation, sample variance, minimum, first $\left(1^{\text {st }}\right)$ quartile $(1 Q)$, median, third $\left(3^{\text {rd }}\right)$ quartile (3Q), maximum, and the count (i.e., number of images processed) for samples PC1N3 and W1N10, their specimens, and their mosaics are reported. Table 3-1 reports the descriptive statistics for PC1N3 and Table 3-2 for W1N10.

From Table 3-1 it can be observed that the means for the mosaics are generally consistent ranging from $80.2 \%$ to $98.5 \%$, where these values were from specimen PC1N3-02, mosaic IV and III, respectively. The standard error ranged from $0.5 \%$ to $5.0 \%$ which also correspond to mosaics III and IV from specimen PC1N3-02, respectively. The standard deviation ranged from $1.5 \%$ to $14.9 \%$, which correspond to PC1N3-02 mosaics III and IV, respectively. Sample variance ranged from $0 \%$ to $2.2 \%$, these values corresponded 
to PC1N3-02 mosaics III and IV, respectively. For the overall sample, the mean was about $92.1 \%$, with a standard error of $1.0 \%$, a standard deviation of $8.1 \%$, and a sample variance of $0.7 \%$.

For sample W1N10, in Table 3-2 it can be observed that the means for the mosaics are fairly consistent ranging from $68.7 \%$ to $97.7 \%$, both of which are part of specimen W1N10-01 mosaic III and IV, respectively. The standard error ranged from $0.5 \%$ to $6.3 \%$ which corresponds to mosaics IV and III, respectively, from specimen W1N10-01. The standard deviation ranged from $1.5 \%$ to $18.8 \%$, which like the standard error corresponded to W1N10-01 mosaic IV and III, respectively. Sample variance ranged from $0 \%$ to $3.5 \%$, these values corresponded to W1N10-01 mosaics IV and III, respectively. For the overall sample, W1N10 had a mean about $88.4 \%$, with a standard error of $1.5 \%$, a standard deviation of $12.6 \%$, and a sample variance of $1.6 \%$.

To summarize,

- Analysis of two (2) specimens per sample show consistent estimation of diatom particle percentages. This indicates that the approach is repeatable and future analysis may only require imaging and analysis of one (1) specimen.

- The range of diatom particle percentage for the Pinecone Way sample is approximately $92 \%$ and $93 \%$. The range of diatom 
particle percentage in the Wikiup Junction sample is approximately $88 \%$ and $89 \%$. 


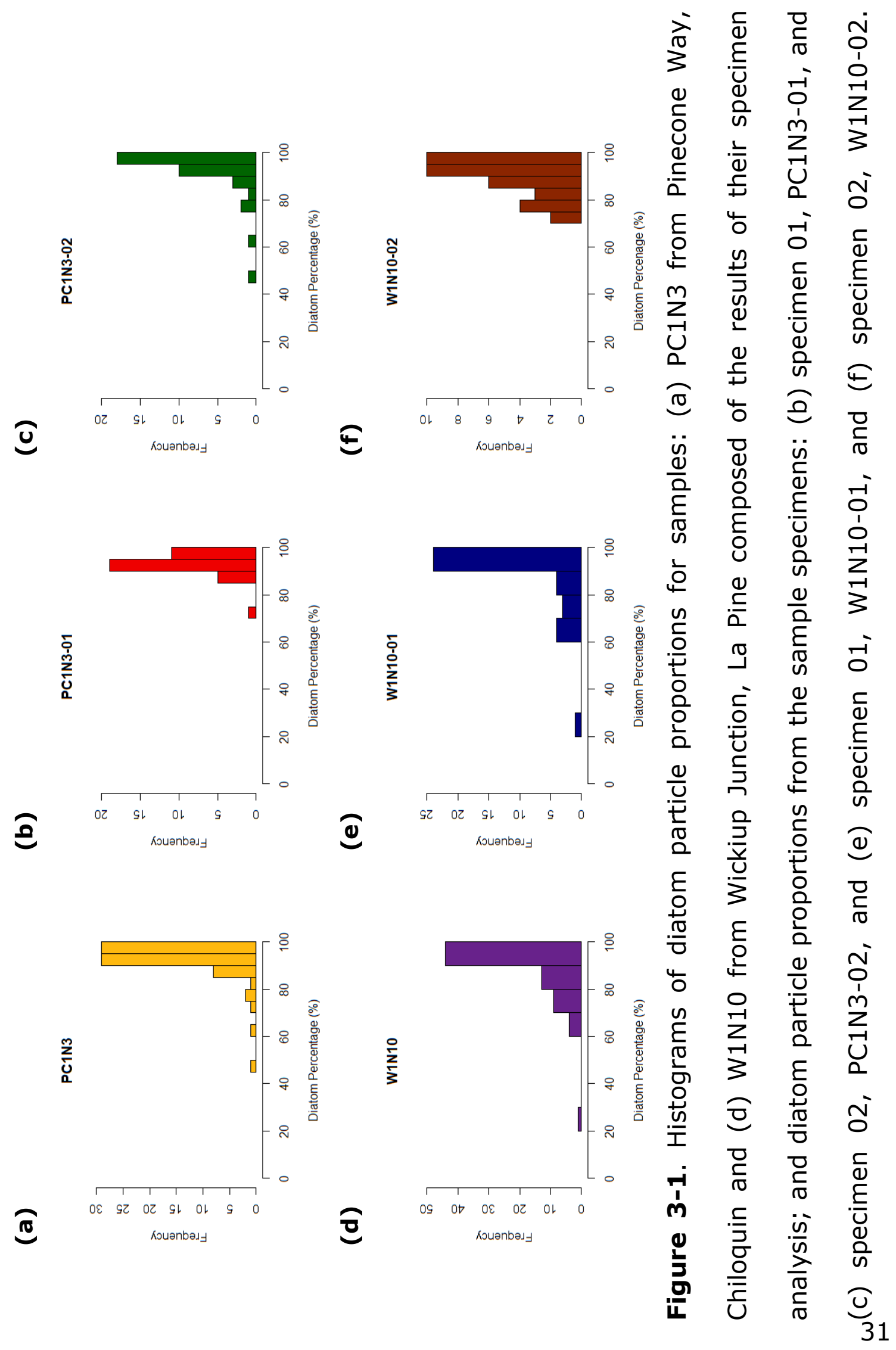




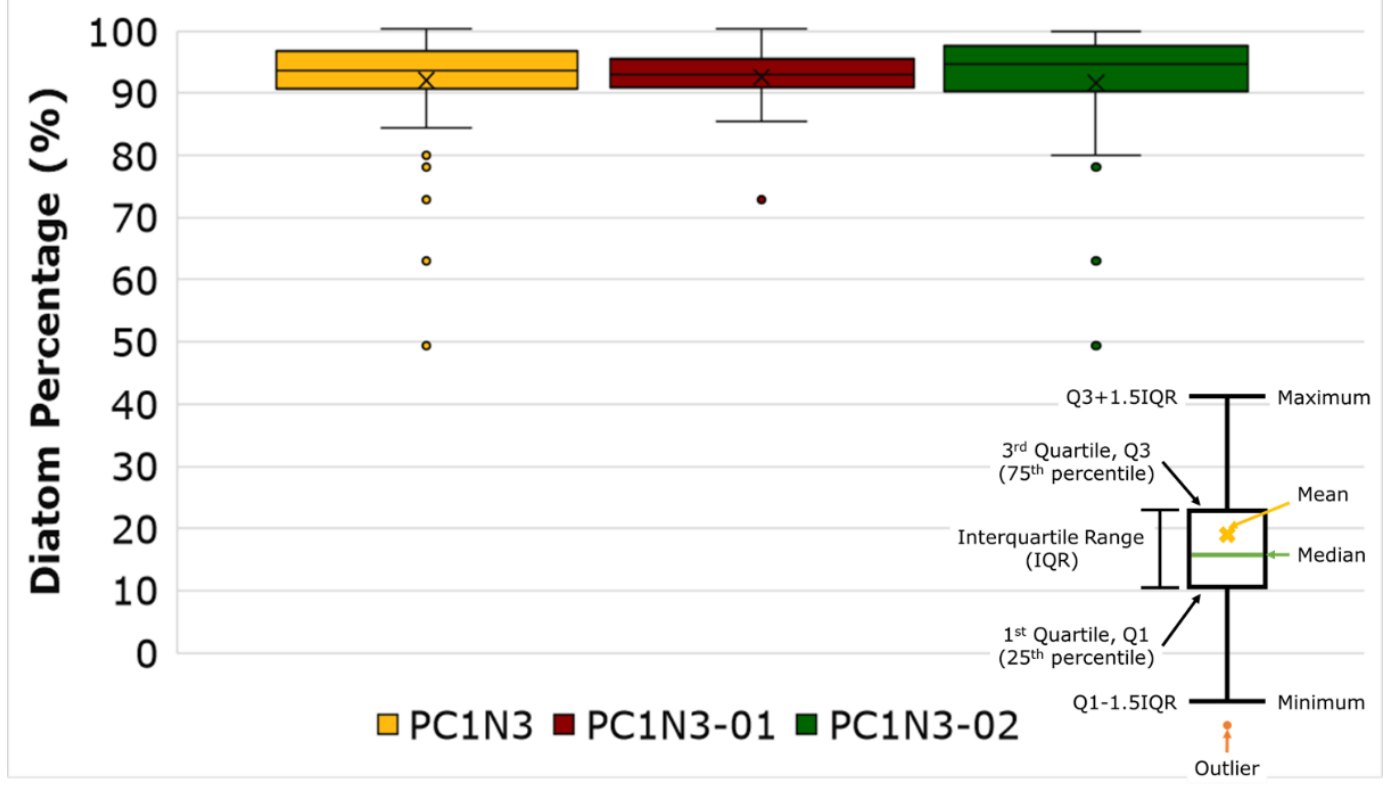

Figure 3-2. Data spread for sample PC1N3 from Pinecone Way, Chiloquin and its two (2) specimens, PC1N3-01 and PC1N3-02.

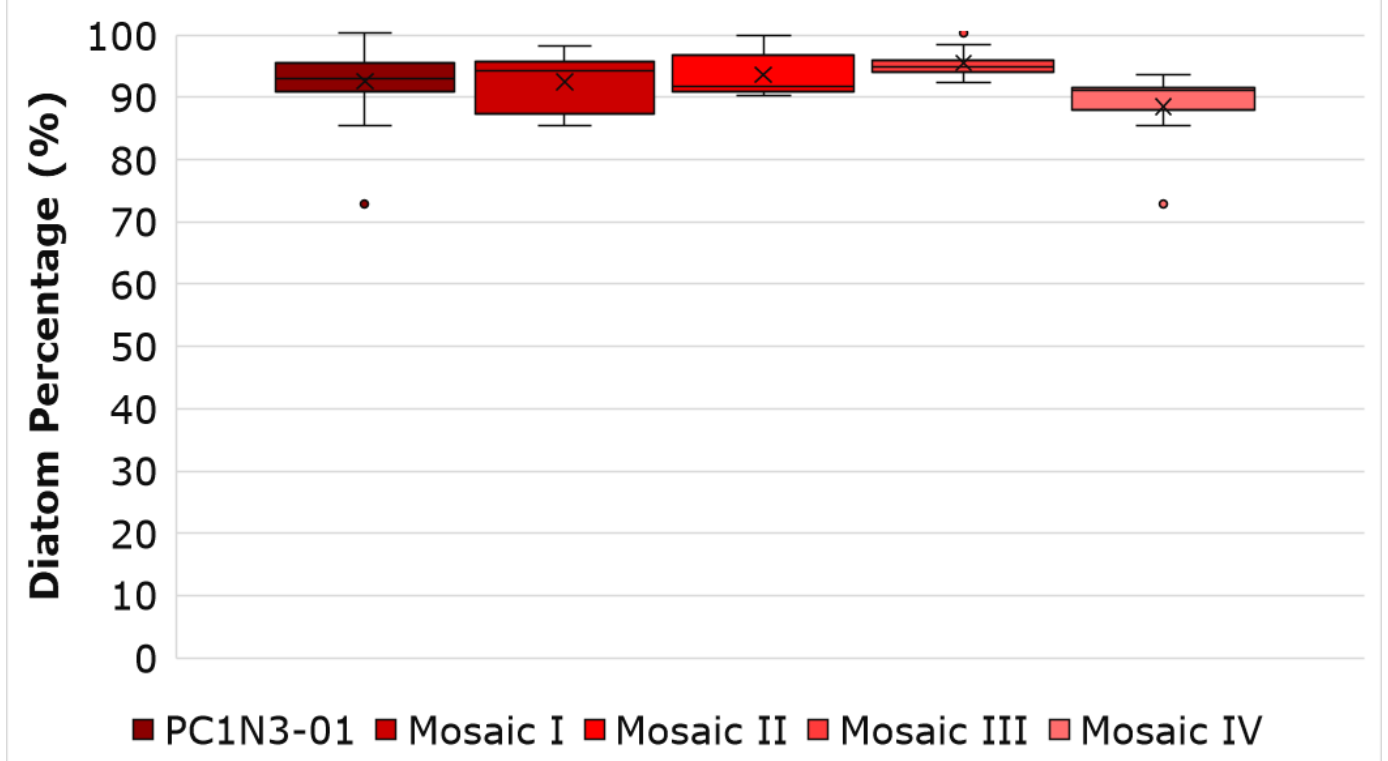

Figure 3-3. Data spread for specimen PC1N3-01 from Pinecone Way, Chiloquin and its four (4) mosaics. 


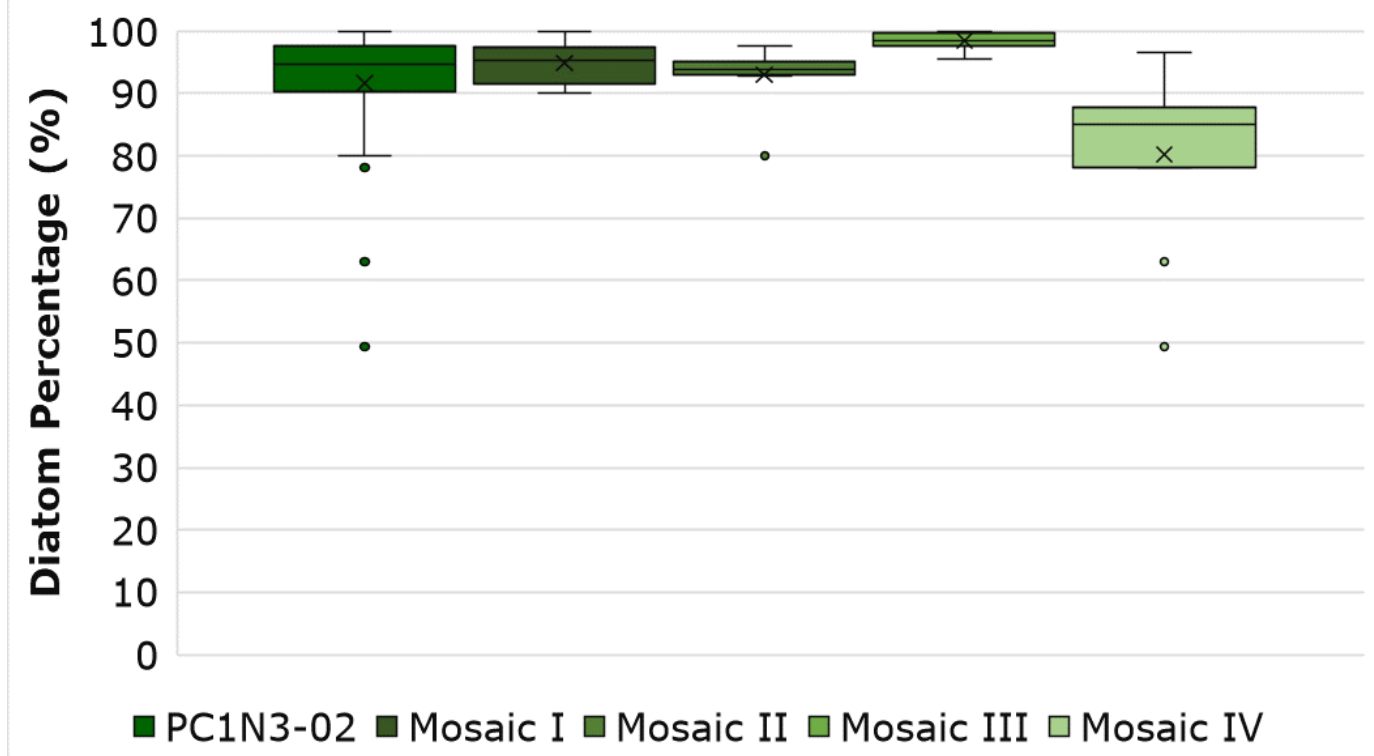

Figure 3-4. Data spread for specimen PC1N3-02 from Pinecone Way, Chiloquin and its four (4) mosaics.

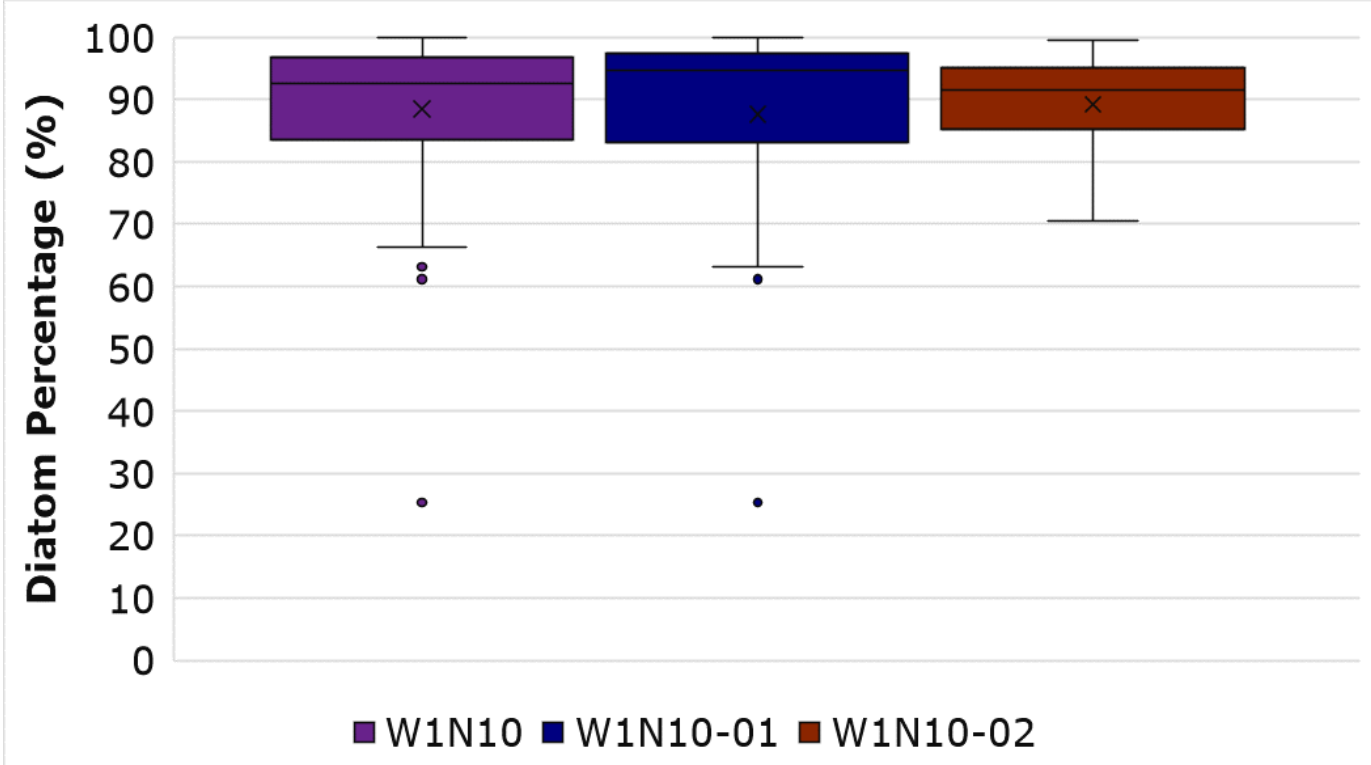

Figure 3-5. Data spread for W1N10 from Wickiup Junction, La Pine and its two (2) specimens, W1N10-01 and W1N10-02. 


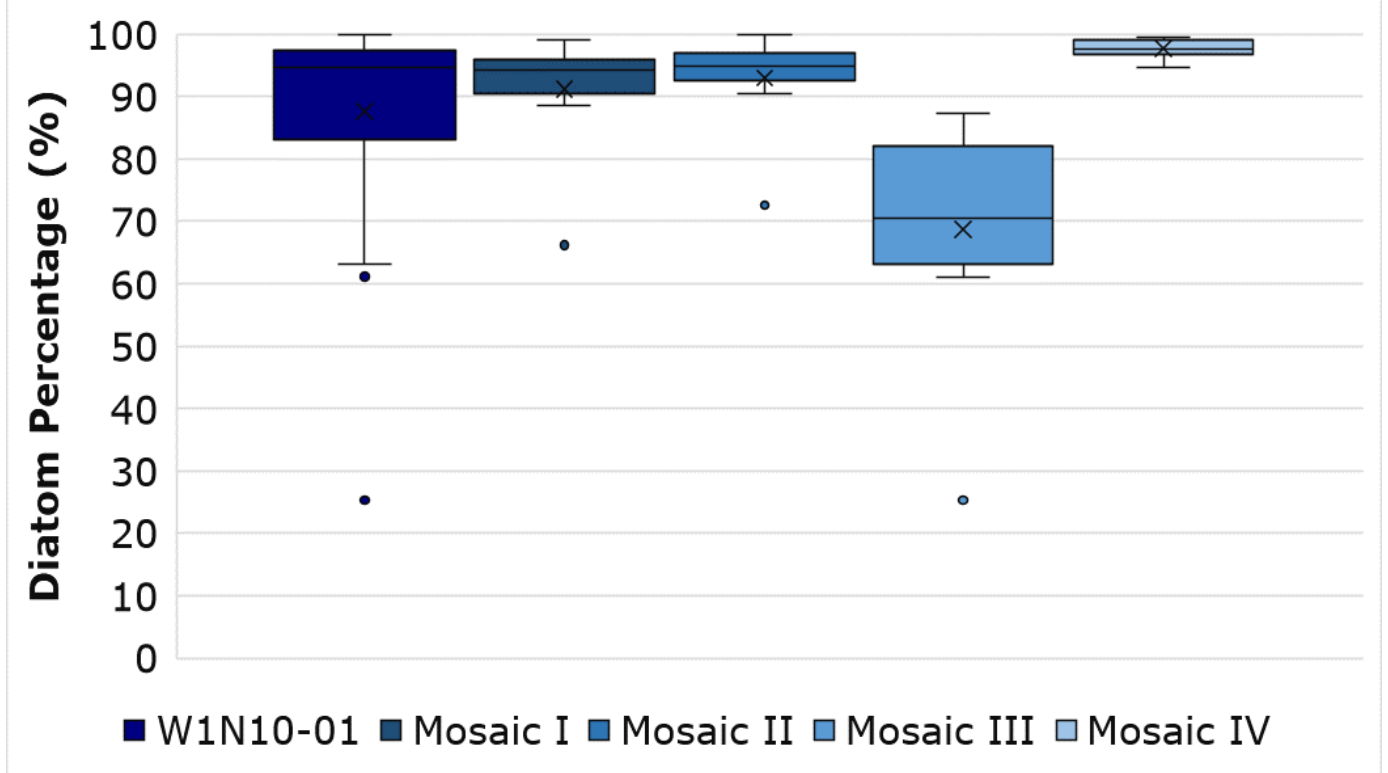

Figure 3-6. Data spread for W1N10-01 from Wickiup Junction, La Pine and its four (4) mosaics.

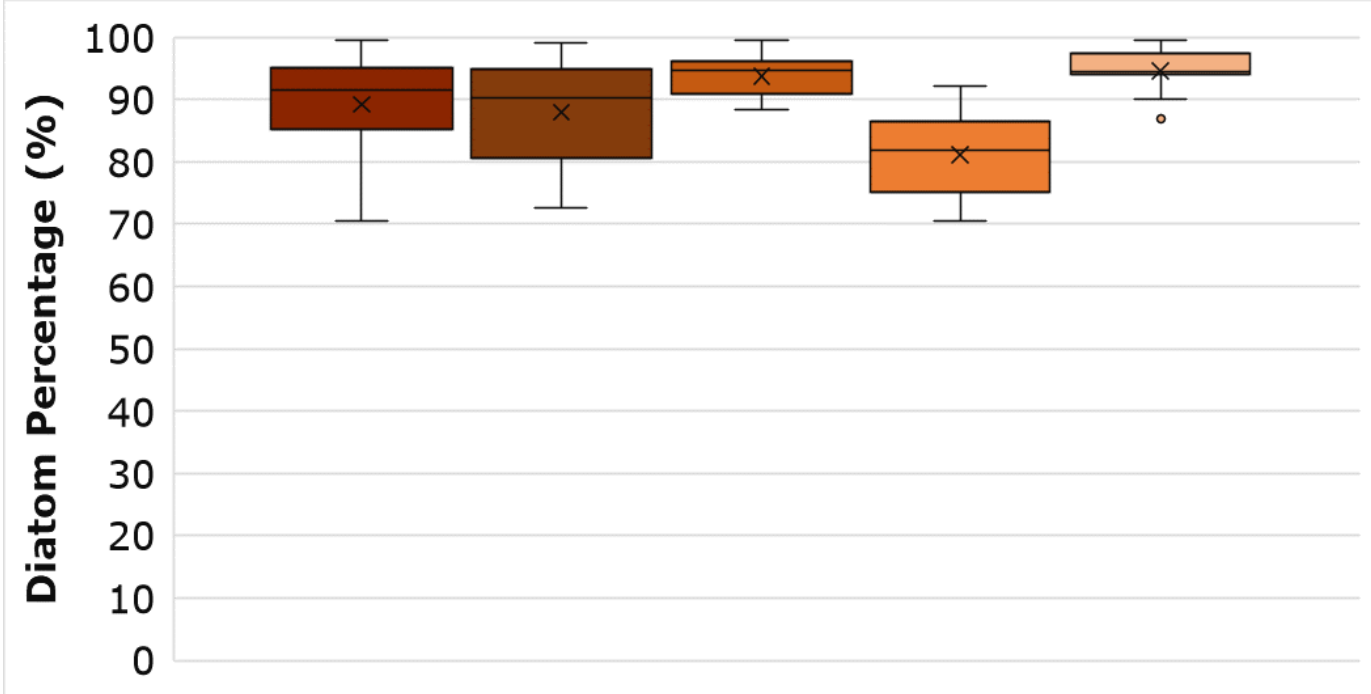

$\square$ W1N10-02 $\square$ Mosaic I $\square$ Mosaic II $\square$ Mosaic III $\square$ Mosaic IV

Figure 3-7. Data spread for W1N10-02 from Wickiup Junction, La Pine and its four

(4) mosaics. 


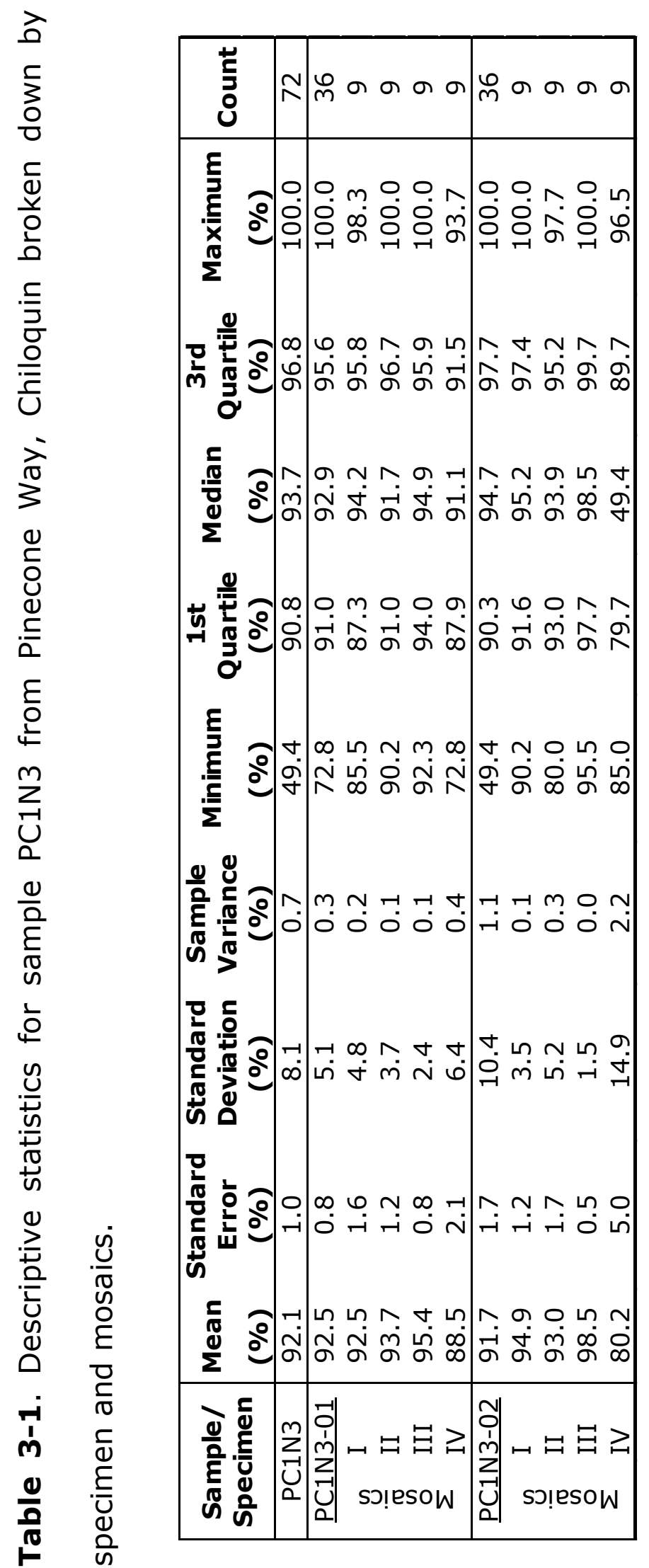


Table 3-2. Descriptive statistics for sample W1N10 from Wickiup Junction, La Pine broken down by specimen and mosaics.

\begin{tabular}{|c|c|c|c|c|c|c|c|c|c|c|}
\hline $\begin{array}{l}\text { Sample/ } \\
\text { Specimen }\end{array}$ & $\begin{array}{l}\text { Mean } \\
(\%)\end{array}$ & $\begin{array}{c}\text { Standard } \\
\text { Error } \\
(\%)\end{array}$ & $\begin{array}{c}\text { Standard } \\
\text { Deviation } \\
(\%)\end{array}$ & $\begin{array}{c}\text { Sample } \\
\text { Variance } \\
(\%)\end{array}$ & $\begin{array}{c}\text { Minimum } \\
(\%)\end{array}$ & $\begin{array}{c}\text { 1st } \\
\text { Quartile } \\
(\%)\end{array}$ & $\begin{array}{c}\text { Median } \\
(\%)\end{array}$ & $\begin{array}{c}\text { 3rd } \\
\text { Quartile } \\
(\%)\end{array}$ & $\begin{array}{c}\text { Maximum } \\
(\%)\end{array}$ & Count \\
\hline W1N10 & 88.4 & 1.5 & 12.6 & 1.6 & 25.3 & 83.5 & 92.6 & 96.8 & 100.0 & 71 \\
\hline W1N10-01 & 87.7 & 2.6 & 15.7 & 2.5 & 25.3 & 83.1 & 94.7 & 97.5 & 100.0 & 36 \\
\hline$I$ & 91.2 & 3.3 & 10.0 & 1.0 & 66.2 & 90.5 & 94.2 & 95.8 & 99.2 & 9 \\
\hline II & 93.0 & 2.7 & 8.2 & 0.7 & 72.6 & 92.6 & 95.0 & 96.9 & 100.0 & 9 \\
\hline III & 68.7 & 6.3 & 18.8 & 3.5 & 25.3 & 63.2 & 70.6 & 82.1 & 87.4 & 9 \\
\hline$\Sigma^{\Sigma} \quad$ IV & 97.7 & 0.5 & 1.5 & 0.0 & 94.7 & 96.8 & 97.6 & 99.0 & 99.5 & 9 \\
\hline W1N10-02 & 89.2 & 1.4 & 8.4 & 0.7 & 70.4 & 85.1 & 91.6 & 95.1 & 99.6 & 35 \\
\hline I & 87.9 & 3.0 & 9.0 & 0.8 & 72.5 & 80.6 & 90.2 & 94.8 & 99.1 & 9 \\
\hline.$\frac{\tilde{U}}{\pi}$ & 93.8 & 1.4 & 4.0 & 0.2 & 88.3 & 90.8 & 94.6 & 96.1 & 99.4 & 8 \\
\hline III & 81.1 & 2.5 & 7.5 & 0.6 & 70.4 & 75.1 & 81.8 & 86.6 & 92.2 & 9 \\
\hline IV & 94.6 & 1.3 & 4.0 & 0.2 & 86.9 & 94.1 & 94.5 & 97.4 & 99.6 & 9 \\
\hline
\end{tabular}




\section{CHAPTER 4: DISCUSSION}

\subsection{Number of Mosaic Images}

Descriptive statistics were used to explore how the number of images making up a mosaic affected the estimates of diatom percentages. The objective of this additional analysis was to investigate how many images should make up a mosaic for repeatable estimation of diatom particle proportion. The presented box-andwhisker plots for specimen W1N10-01 in Figure 4-1, and specimen W1N10-02 in Figure 4-2 explore the spread of diatom particle proportion when the analysis was performed with mosaics of four-byfour $(4 \times 4)$, three-by-three $(3 \times 3)$, two-by-two $(2 \times 2)$, and one-by-one (1x1) image. From W1N10-01, mosaic IV was used, and from W1N1002, mosaic II was used. All images associated with the step-by-step procedure described in CHAPTER 2:, are presented in Appendix B, demonstrating the selected images for the various sized mosaics. Two (2) different mosaics from two (2) different specimens were selected due to how populated one was over the other, i.e., W1N10-01 mosaic IV has more particles while W1N10-02 mosaic II has fewer particles.

From the box-and-whisker plots it can be observed that the data for the $4 \times 4$ mosaics for both specimens have the largest spread with W1N10-01 having its minimum at $80.6 \%$ and its maximum at $99.5 \%$ (Figure 4-1); while W1N10-02 has its minimum at $43.2 \%$ and its 
maximum at $99.4 \%$ (Figure $4-2$ ). However, it should be noted that of the two (2) specimens W1N10-02 has its minimum as an outlier, since it is more than 1.5 times the length of the IQR and drives its mean to be lower than its median. The spread of the data for the $3 \times 3,2 \times 2$, and 1X1 image for both W1N10-01 mosaic IV and W1N10-02 mosaic II decreases with decreasing number of images.

Table 4-1, presents the associated descriptive statistics such as the mean, standard error, standard deviation, sample variance, minimum, first $\left(1^{\text {st }}\right)$ quartile, $\mathrm{Q} 1$, median, third $\left(3^{\text {rd }}\right)$ quartile, Q3, maximum, and the count (i.e., number of images processed) for each mosaic of each specimen. The mean and median for the $4 \times 4,3 \times 3$, and $2 \times 2$ mosaics are within one standard deviation of each other for both W1N10-01 mosaic IV and W1N10-02 mosaic II (Table 4-1). The mean and median for W1N10-01 increased with decreasing mosaic size from $93.3 \%$ to $98.6 \%$ and $96.7 \%$ to $98.8 \%$ (there was no median for the one image), respectively; while for W1N10-02 the mean increased then decreased with decreasing mosaic size from $82.5 \%$ to $95.2 \%$ and then to $88.5 \%$, and the median increased with decreasing size from $88.5 \%$ to $96.5 \%$ (there was no median for the one image). The standard error of the $4 \times 4,3 \times 3$, and $2 \times 2$ mosaics was less than $1.7 \%$ for W1N10-01 mosaic IV, while it ranged from 1.4 to $4.5 \%$ for W1N1002 mosaic II. The standard deviations for W1N10-01 mosaic IV 
decrease with decreasing number of images per mosaic, from $6.9 \%$ to $0.8 \%$, and it ranged from $4.0 \%$ to $17.4 \%$ for W1N10-02 mosaic II. Sample variance was particularly small for W1N10-01 at $0.5 \%$ for the $4 \times 4$ mosaic and $0.0 \%$ for both the $3 \times 3$ and $2 \times 2$ mosaic. For $\mathrm{W} 1 \mathrm{~N} 10-02$ the sample variance was $3.0 \%$ for the $4 \times 4$ mosaic, $9.9 \%$ for the $3 \times 3$ mosaic, and $0.2 \% 2 \times 2$ mosaic.

Something to note is that there was an image from W1N10-02 mosaic II excluded from this analysis due to the lack of information it presented, i.e., the image had no particles. This demonstrates the importance of choosing a populated area within a specimen quadrant during the imaging process and having more than one mosaic per specimen as one image could greatly influence the resulting diatom percentages.

Overall, using $3 \times 3$ image mosaics for this investigation was a reasonable approach since it provided a larger area to analyze than the smaller mosaics, repeatable results, and required less time to do image processing than a $4 \times 4$ image mosaic. Additionally, it appears that the spread of data for $4 \times 4$ image mosaics becomes larger than for $3 \times 3$ mosaics. This is likely an artifact of selecting the $3 \times 3$ image mosaics as the highest quality block of images and with the most soil particles. Conversely, with $4 \times 4$ image mosaics, this may include analysis of images with very few soil particles or relatively low-quality 
images. Therefore, it may be beneficial to choose nine (9) greatly populated images from the 16 images making up a mosaic, or quadrant, without the condition that they must form a mosaic as it would ensure that every image processed would be representative of the soil specimen and thus the soil sample.

\subsection{Relationship Between Geotechnical Properties and Diatom Percentage}

To investigate the relationship between geotechnical properties such as the liquid limit ( $L L)$ and the plastic limit ( $P L)$ the plasticity charts published by Shiwakoti et al. (2002), and Al Shatnawi and Bandini (2018), included here as Figure 1-4 and Figure 1-5, respectively, were adapted to include the $\mathrm{LL}$ and $\mathrm{PL}$ of the sample PC1N3 from the Pinecone Way field site against its mean diatom percentage of $92 \%$. The LL and PL for PC1N3 were provided by Jiayao Wang, Ph.D. candidate at Oregon State University (OSU). Figure 4-3 present the LL data and Figure 4-4 the PL data. There was no available LL and PL data for W1N10 from the Wickiup Junction, La Pine field site.

In both figures, it can be observed that the large $\mathrm{LL}$ and $\mathrm{PL}$ values, $109.4 \%$ and $74.7 \%$, respectively, agree with the general trend of increasing LL and PL with increasing diatom content. 


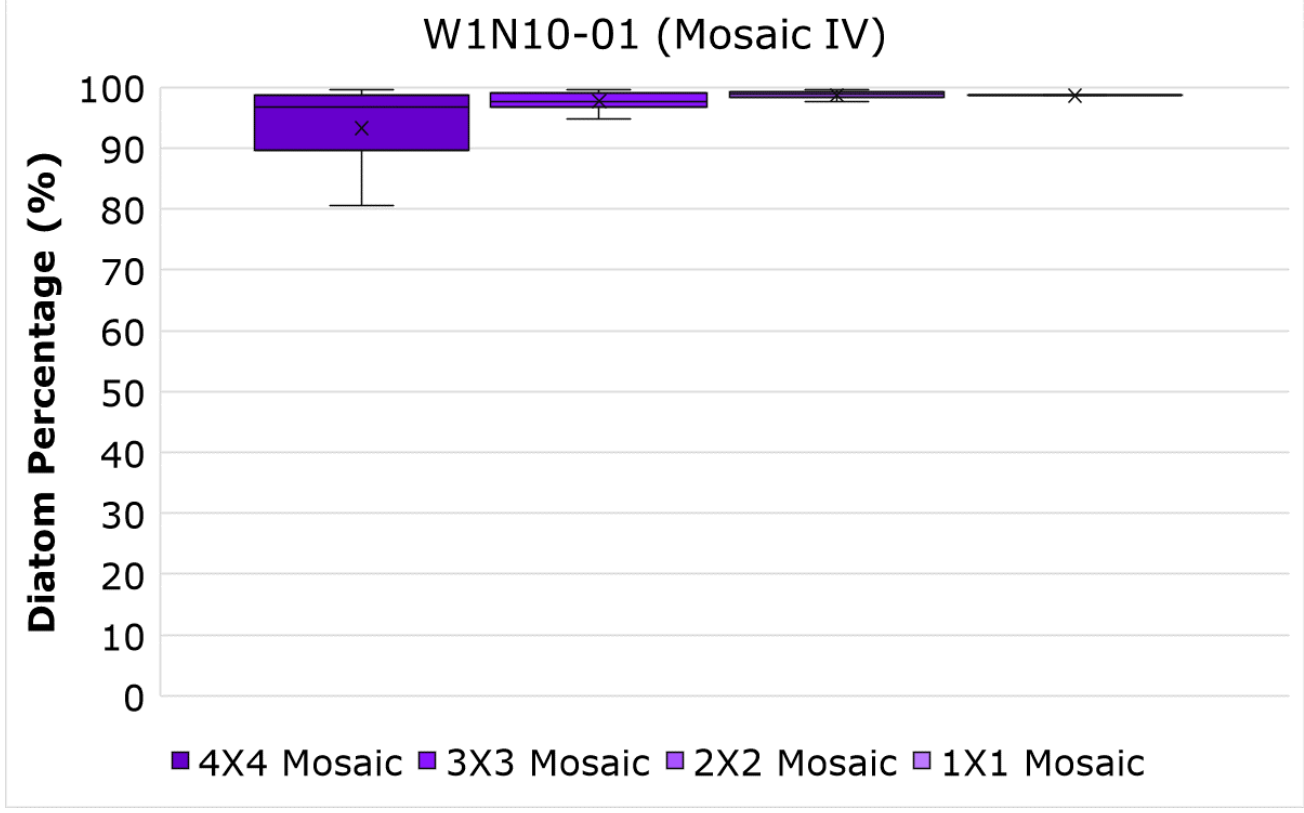

Figure 4-1. Diatom percentages estimated from images making up mosaic IV for the specimen W1N10-01 from Wickiup Junction.

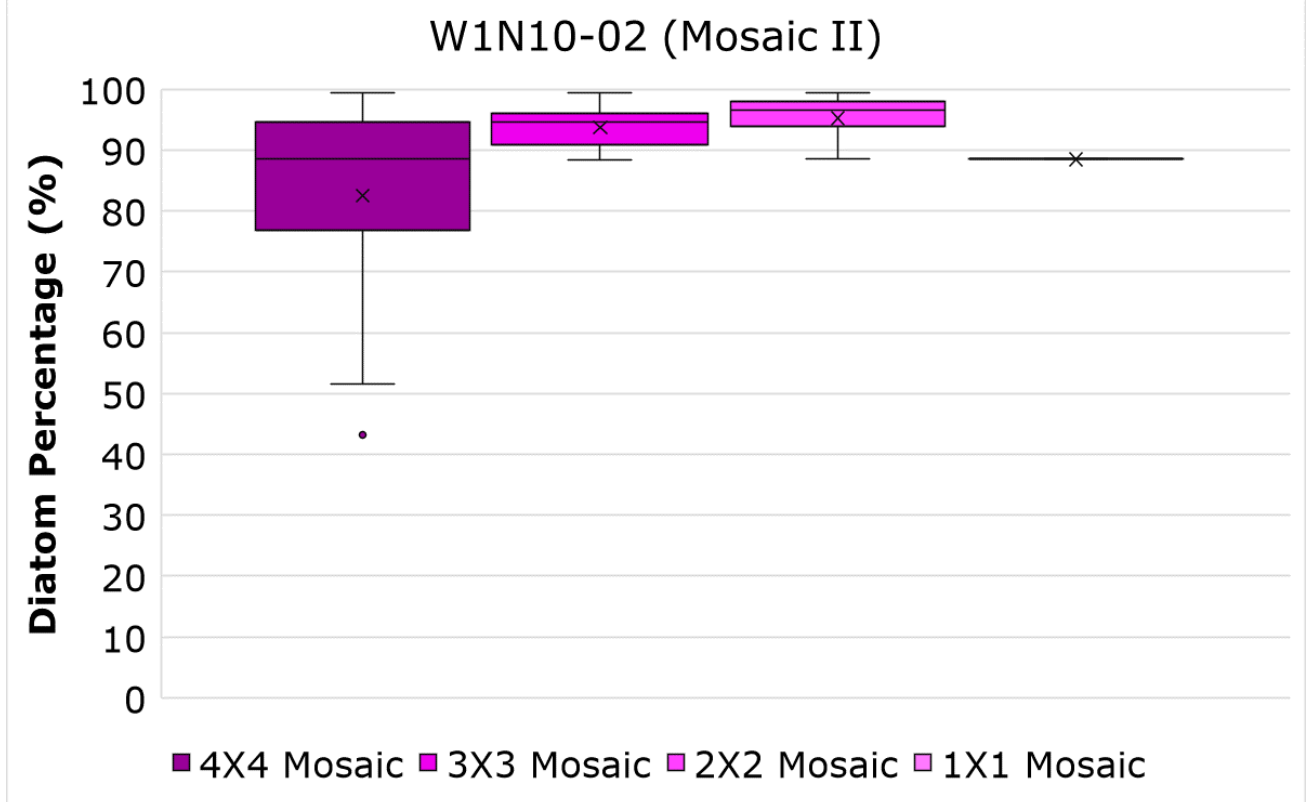

Figure 4-2. Diatom percentages estimated from images making up mosaic II for the specimen W1N10-02 from Wickiup Junction. 
Table 4-1. Descriptive statistics for mosaic IV from specimen W1N1001 and mosaic II from specimen W1N10-02, both from Wickiup Junction. The mean values presented for the one image (1) are the raw estimated values of diatom percentages from each image.

\begin{tabular}{|c|c|c|c|c|c|c|c|c|}
\hline Specimen & & W1I & $0-01$ & & & W1I & $0-02$ & \\
\hline Mosaic & & & & & & & & \\
\hline Size & $4 \times 4$ & $3 \times 3$ & $2 \times 2$ & $1 \times 1$ & $4 \times 4$ & $3 \times 3$ & $2 \times 2$ & $1 \times 1$ \\
\hline Mean & 93.3 & 97.7 & 98.7 & 98.6 & 82.5 & 93.8 & 95.2 & 88.5 \\
\hline Standard Error & 1.7 & 0.5 & 0.4 & - & 4.5 & 1.4 & 2.4 & - \\
\hline Standard Deviation & 6.9 & 1.5 & 0.8 & - & 17.4 & 4.0 & 4.8 & - \\
\hline Sample Variance & 0.5 & 0.0 & 0.0 & - & 3.0 & 0.2 & 0.2 & - \\
\hline Minimum & 80.6 & 94.7 & 97.6 & - & 43.2 & 88.3 & 88.5 & - \\
\hline 1st Quartile & 89.5 & 96.8 & 98.4 & - & 76.6 & 90.8 & 93.8 & - \\
\hline Median & 96.7 & 97.6 & 98.8 & - & 88.5 & 94.6 & 96.5 & - \\
\hline 3rd Quartile & 99.0 & 99.0 & 99.2 & - & 94.5 & 96.1 & 97.9 & - \\
\hline Maximum & 99.5 & 99.5 & 99.5 & - & 99.4 & 99.4 & 99.4 & - \\
\hline Count & 16 & 9 & 4 & - & 15 & 8 & 4 & - \\
\hline
\end{tabular}




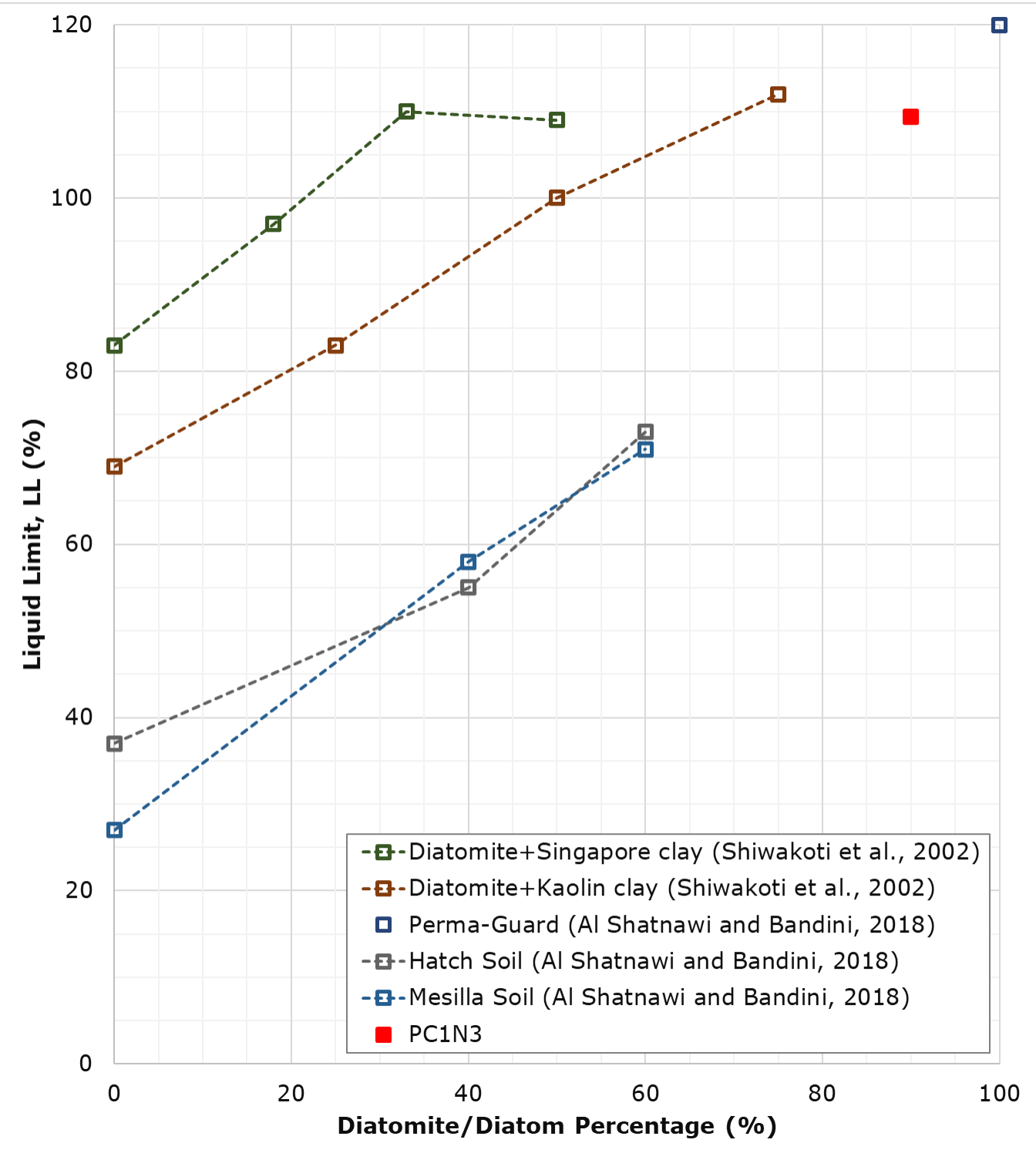

Figure 4-3. Liquid limit (LL) data published by Shiwakoti et al. (2002) and Al Shatnawi and Bandini (2018) adapted to include the LL of sample PC1N3 from the Pinecone Way, Chiloquin field site courtesy of Jiayao Wang (Ph.D. candidate, OSU). 


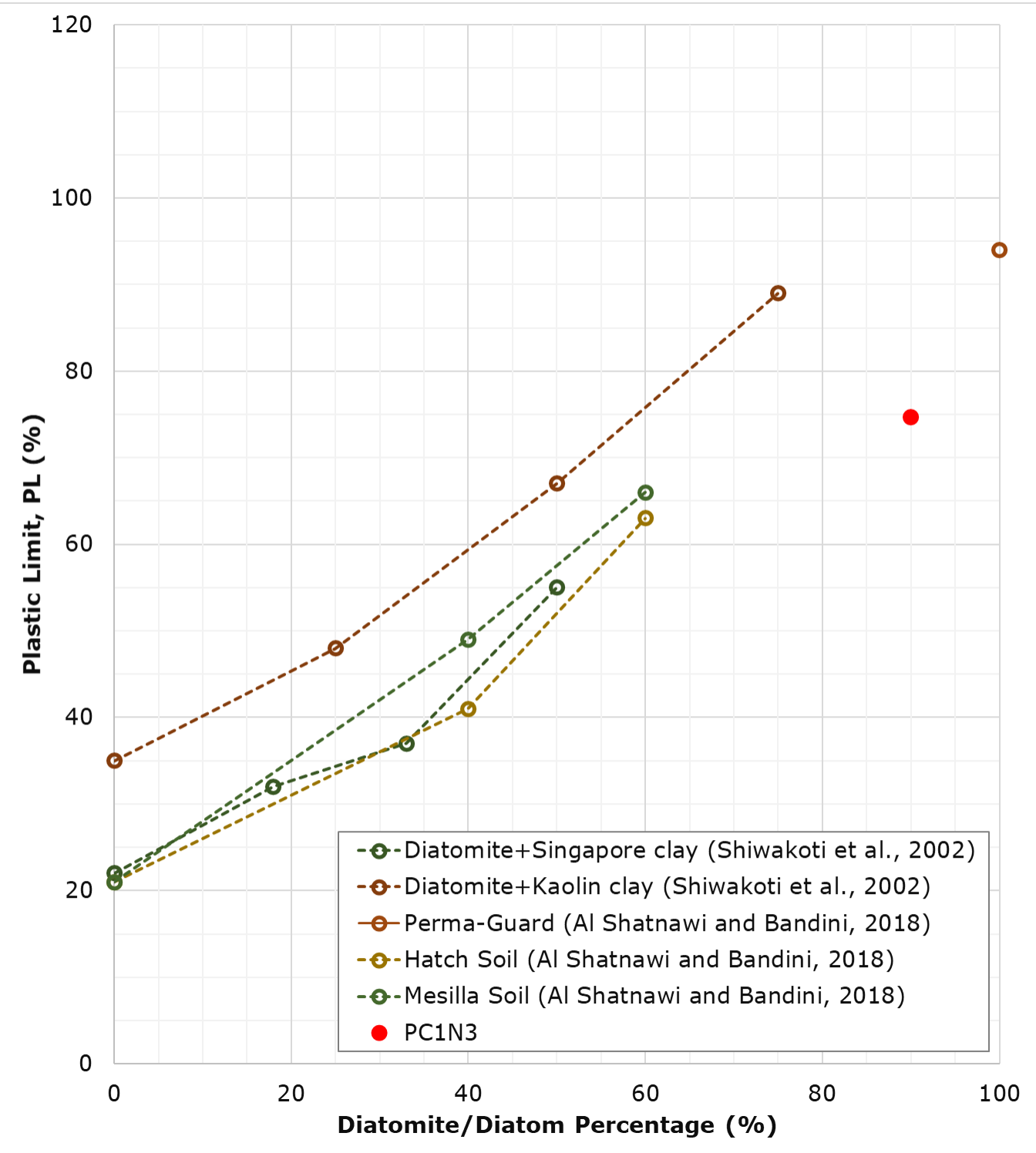

Figure 4-4. Plastic limit (PL) data published by Shiwakoti et al. (2002) and Al Shatnawi and Bandini (2018) adapted to include the PL of sample PC1N3 from the Pinecone Way, Chiloquin field site courtesy of Jiayao Wang (Ph.D. candidate, OSU). 


\section{CHAPTER 5: CONCLUSIONS AND FUTURE RECOMMENDATIONS}

\subsection{Conclusions}

This study presented a method to estimate diatom particle proportion in natural soils. The method uses quantitative stereology analysis on high resolution SEM $3 \times 3$ image mosaics at 3000 magnification. Quantitative stereology analysis of the images provides an estimation of diatom particle proportion within all soil particles present.

This study applied the method to two (2) natural diatomaceous soil samples from southern and central Oregon. From each sample, two (2) specimens were prepared, imaged, and analyzed to (i) estimate their diatom particle proportions, and (ii) evaluate the repeatability of the analysis approach. The results indicated that the method provides reasonable and repeatable estimates of diatom particle proportions. For future analysis, if multiple specimens are not feasible, it is recommended to capture images from more than one populated area per specimen and carefully selected the images with the most particles present to reduce the possibility that any one image within the captured area greatly influences the resulting diatom percentage of said area.

The analysis of two (2) samples estimated a diatom particle proportion of approximately $92 \%$ to $93 \%$ from the Pinecone Way 
sample, and approximately $88 \%$ to $89 \%$ from the Wikiup Junction sample.

This study also preliminarily examined how diatom particle proportions of these natural soils affects soil properties. The liquid and plastic limit of the sample from the Pinecone Way field site agrees with the published data of laboratory diatom mixtures where increasing the diatom percentage increased the resulting liquid and plastic limit values.

\subsection{Future Recommendations}

Future recommendations for this work include (i) additional validation of the approach with laboratory-prepared soil mixtures, (ii) consideration of other statistical tools, and (iii) continued analysis of diatomaceous samples from the ODOT project SPR-820.

In general, there is benefit in processing SEM images of laboratory mixtures of diatomite and well-studied geotechnical materials such as Kaolin clay and silica silt with the presented quantitative stereology approach. In this manner the resulting diatom percentages can be compared to the known diatom percentages (by weight) to validate the procedure presented herein.

Due to the nature of the sample, there is a natural cut-off of the data at $100 \%$ diatom content, it is of interest to investigate the 
possibility of using statistical tools for normal distribution on this data to compare diatom percentages from various field sites obtained at different depths and investigate differences in their results.

Characterization of engineering properties of Oregon's diatomaceous soils is ongoing. Future work will continue image analysis of these soils to estimate diatom particle proportions, and then relate these diatom particle proportions to engineering properties including: index properties, strength parameters, and compressibility. 


\section{REFERENCES}

1. Al Shatnawi, H.H. and Bandini, P. (2018) Limitations of Classification for Soils that Contain Diatom Microfossils. IFCEE, $123-132$.

2. Bradbury, J. (2004). Nature's nanotechnologists: Unveiling the secrets of diatoms. PLoS Biology, 2, $1512-1515$.

3. Caicedo, B., Mendoza, C., López, F., and Lizcano, A. (2018). Behavior of diatomaceous soil in lacustrine deposits of Bogotá, Colombia. Journal of rock mechanics and geotechnical engineering, 10, $367-379$.

4. Díaz-Rodríguez, J. A., and López-Molina, J. A. (2009). Cyclic behavior of diatomaceous soils. In Proceedings of the 17 th International Conference on Soil Mechanics and Geotechnical Engineering: The Academia and Practice of Geotechnical Engineering, Alexandria, Egypt, 1, 159 - 162.

5. Evans, T.M. (2005). Microscale physical and numerical investigations of shear banding in granular soils. PhD Thesis, Georgia Institute of Technology, Atlanta.

6. Evans, T.M., and Frost, J.D. (2010). Multiscale investigation of shear bands in sand: Physical and numerical experiments. International Journal for Numerical and Analytical Methods in Geomechanics, 34, $1634-1650$. 
7. Hardwood, D.H. (1999). Diatomite. The Diatoms: Applications for the Environmental and Earth Sciences, Cambridge University Press, $437-443$.

8. Kale, A, and Karthick, B. (2015). The diatoms: Big significance of tiny glass houses. Resonance: Journal of Science Education, 20, $919-930$.

9. Locat, J., Tanaka, H., Tan, T.S., Dasari, G.R., and Lee, H. (2013). Natural soils: geotechnical behavior and geological knowledge. Characterisation and Engineering Properties of Natural Soils, 1, 3 - 28.

10. Round, F.E., Crawford, R.M., and Mann, D.G. (1990). The Diatoms: Biology and Morphology of the Genera. Cambridge University Press.

11. Shiwakoti, D.R., Tanaka, H., Tanaka, M., and Locat, J., 2002. Influences of diatom microfossils on engineering properties of soils. Soils and Foundations, 42, 1 - 17.

12. Underwood, E.E. (1970). Quantitative Stereology, AddisonWesley Publishing Company, Reading, MA. 
APPENDIX A: MOSAICS 


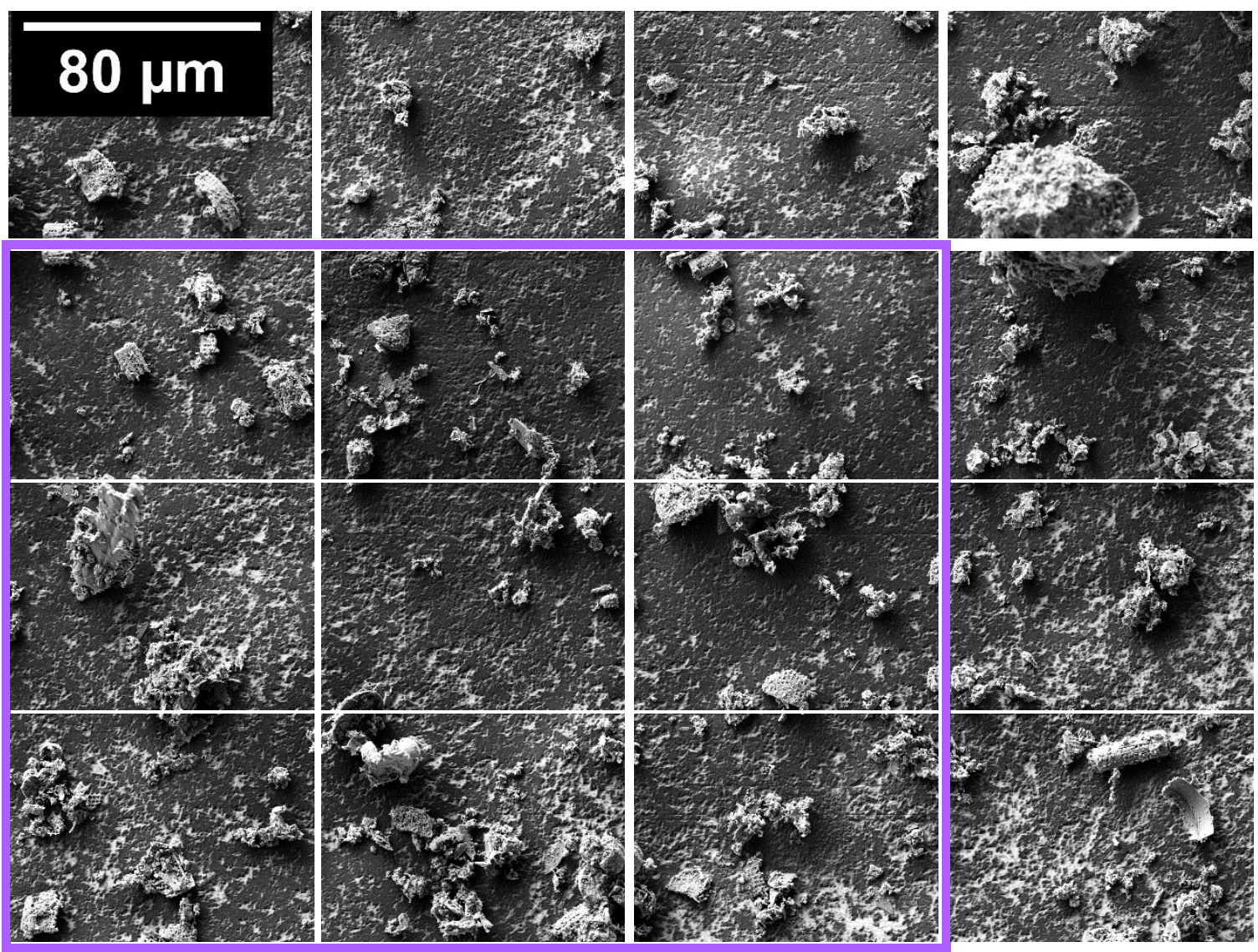

Figure 5-1. Four-by-four (4x4) image mosaic of original SEM images from specimen 01 from sample PC1N13 from the Pinecone Way, Chiloquin field site. The purple box illustrates the chosen three-bythree ( $3 \times 3$ ) image mosaic chosen for analysis, Mosaic I. Images were captured at a magnification of 3000, and the scale bar of $80 \mu \mathrm{m}$ on the top right image applies for all images. 


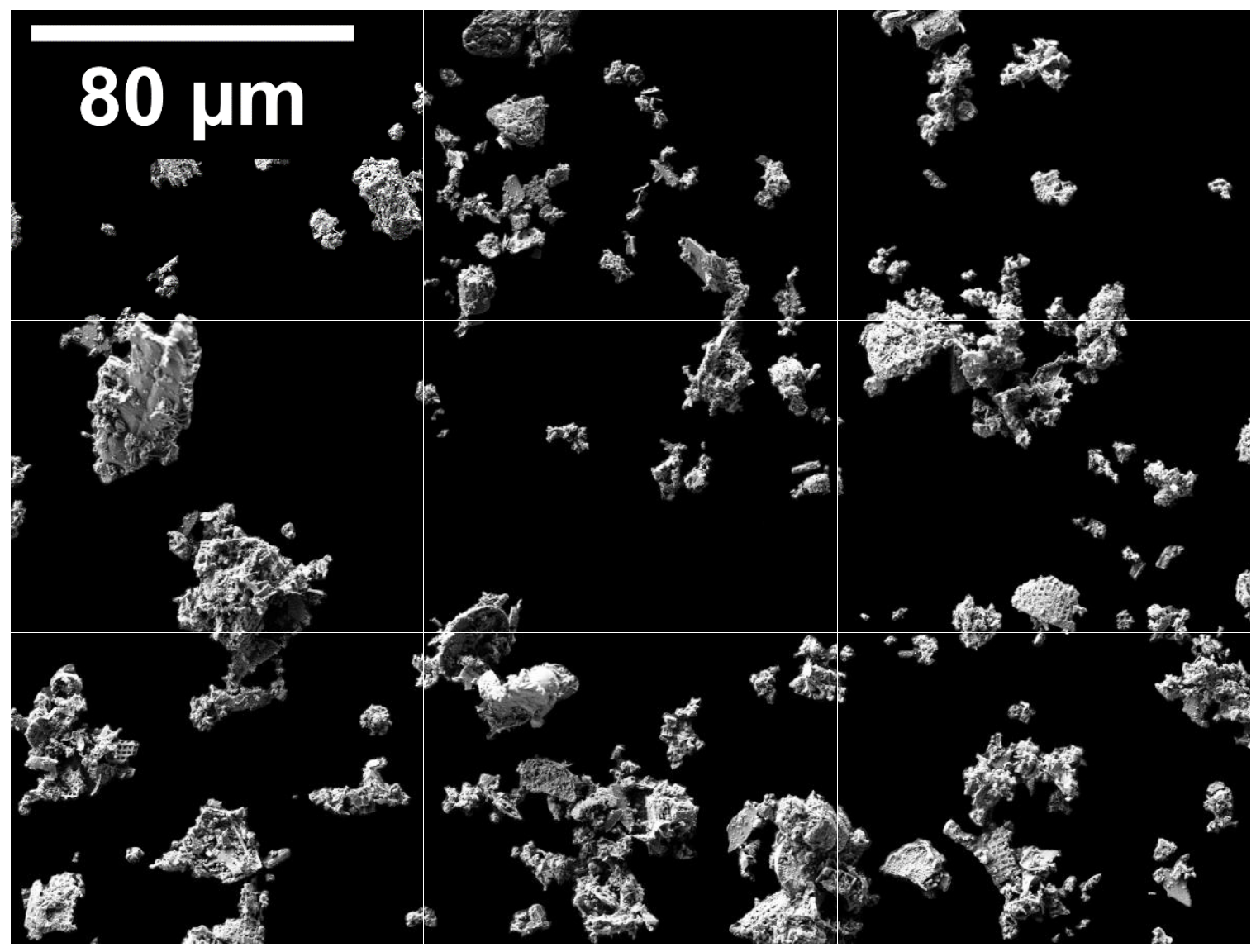

Figure 5-2. Mosaic I, $3 \times 3$ image mosaic of SEM images with all particles on a black background from specimen 01 from sample PC1N13 from the Pinecone Way, Chiloquin field site. Images were captured at a magnification of 3000, and the scale bar of $80 \mu \mathrm{m}$ on the top right image applies for all images. 


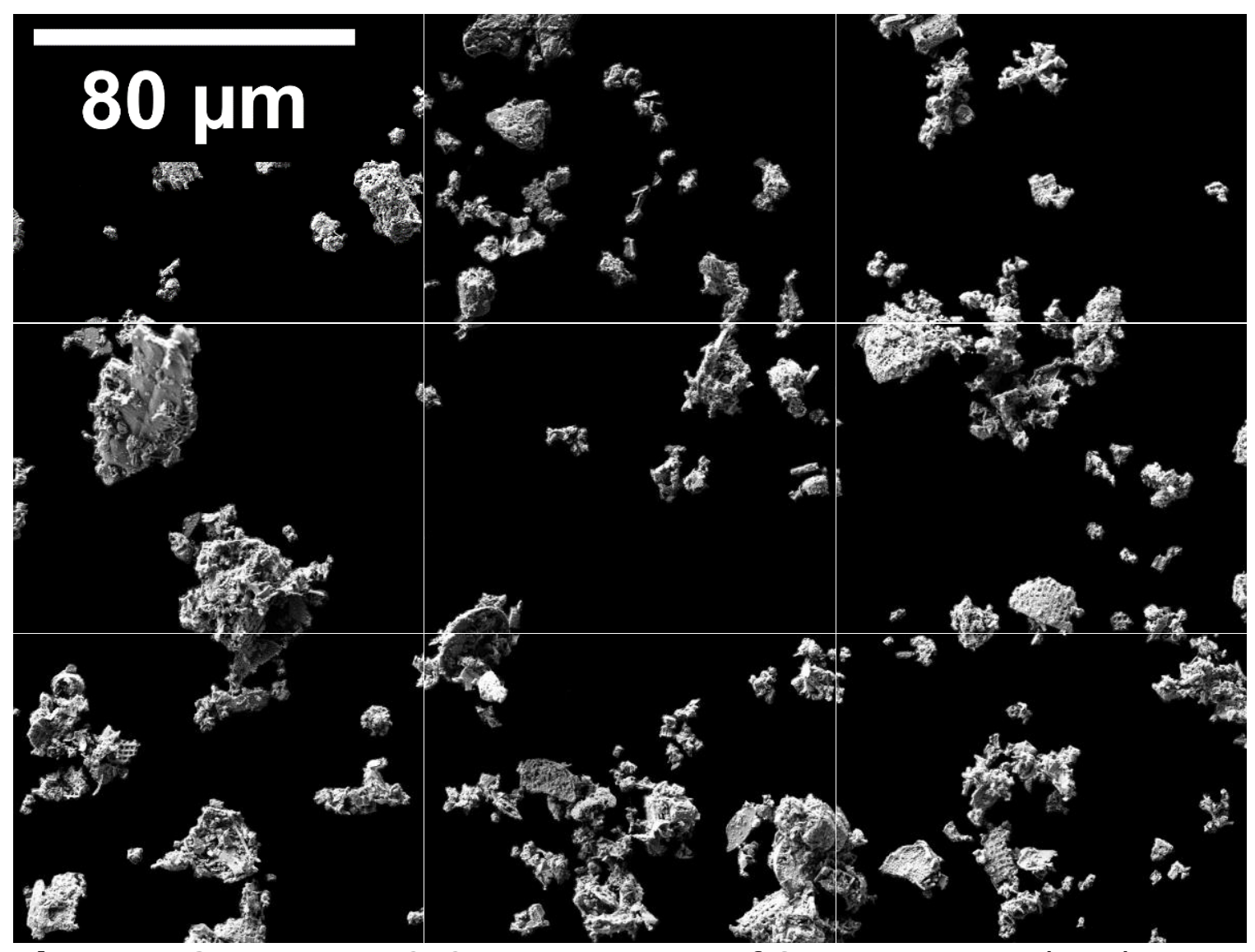

Figure 5-3. Mosaic I, $3 \times 3$ image mosaic of SEM images with only diatom particles on a black background from specimen 01 from sample PC1N13 from the Pinecone Way, Chiloquin field site. Images were captured at a magnification of 3000, and the scale bar of $80 \mu \mathrm{m}$ on the top right image applies for all images. 


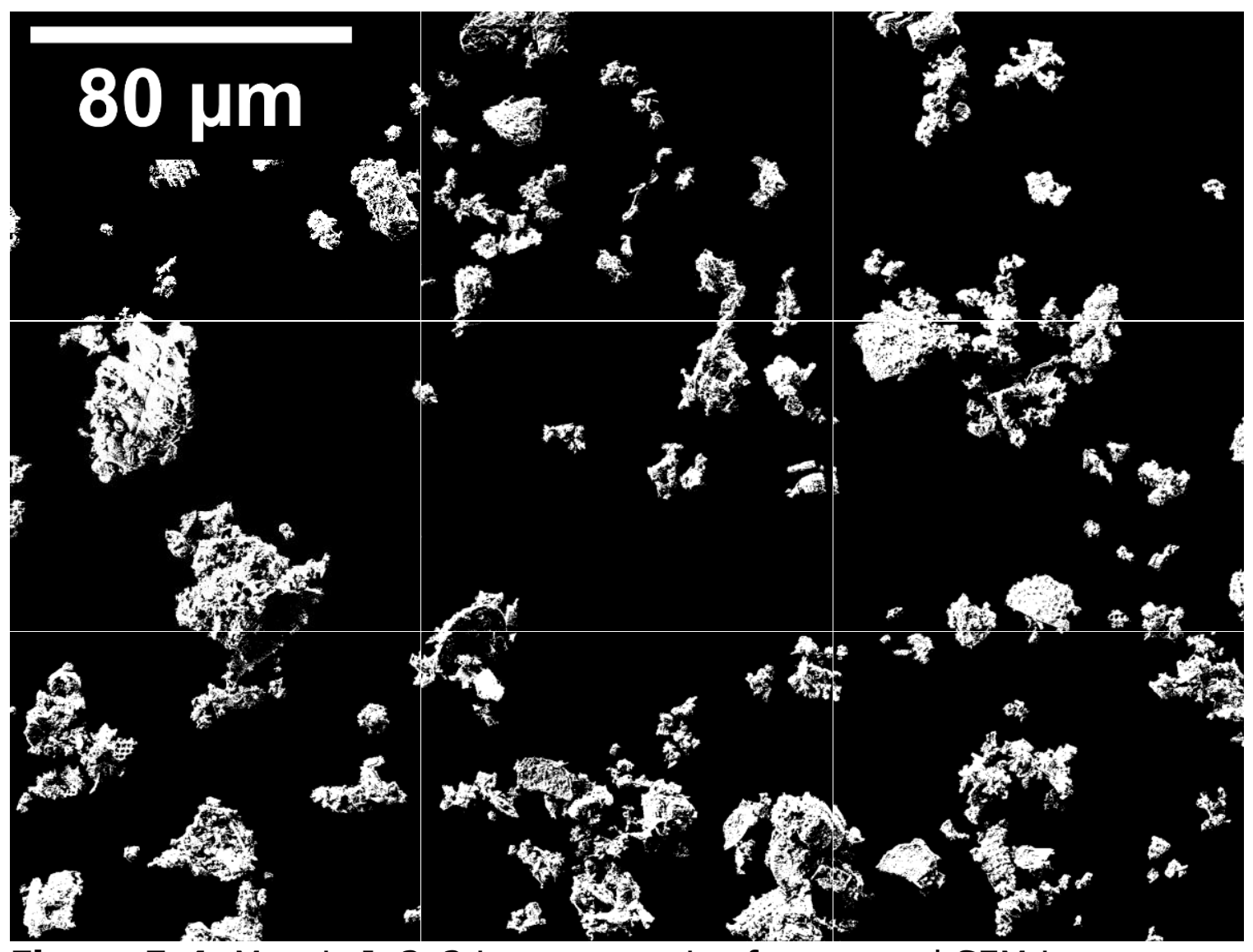

Figure 5-4. Mosaic I, $3 \times 3$ image mosaic of processed SEM images with only diatom particles on a black background from specimen 01 from sample PC1N13 from the Pinecone Way, Chiloquin field site. Images were captured at a magnification of 3000, and the scale bar of $80 \mu \mathrm{m}$ on the top right image applies for all images. 


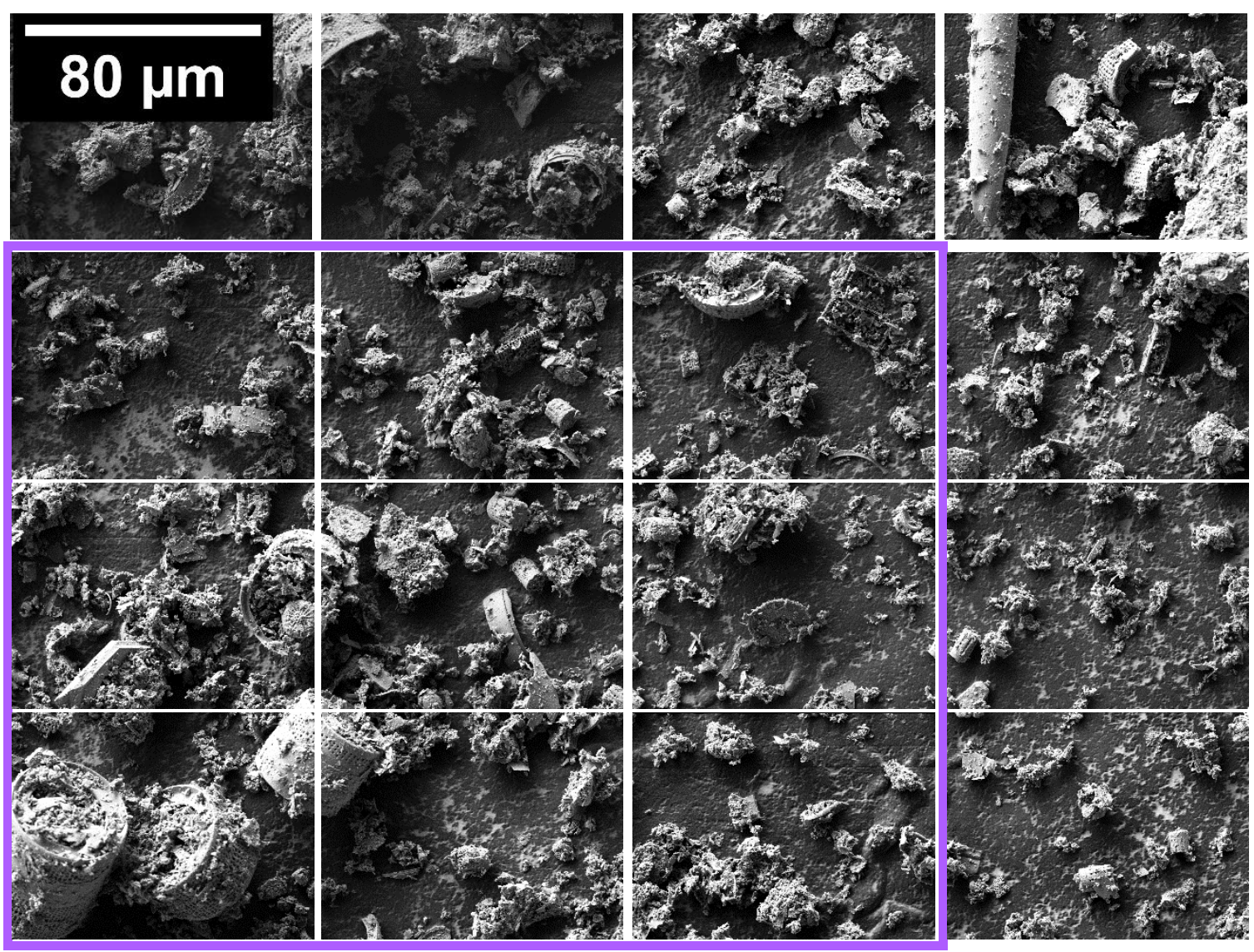

Figure 5-5. $4 \times 4$ image mosaic of original SEM images from specimen 01 from sample PC1N13 from Pinecone Way, Chiloquin field site. The purple box illustrates the chosen $3 \times 3$ image mosaic chosen for analysis, Mosaic II. Images were captured at a magnification of 3000, and the scale bar of $80 \mu m$ on the top right image applies for all images. 


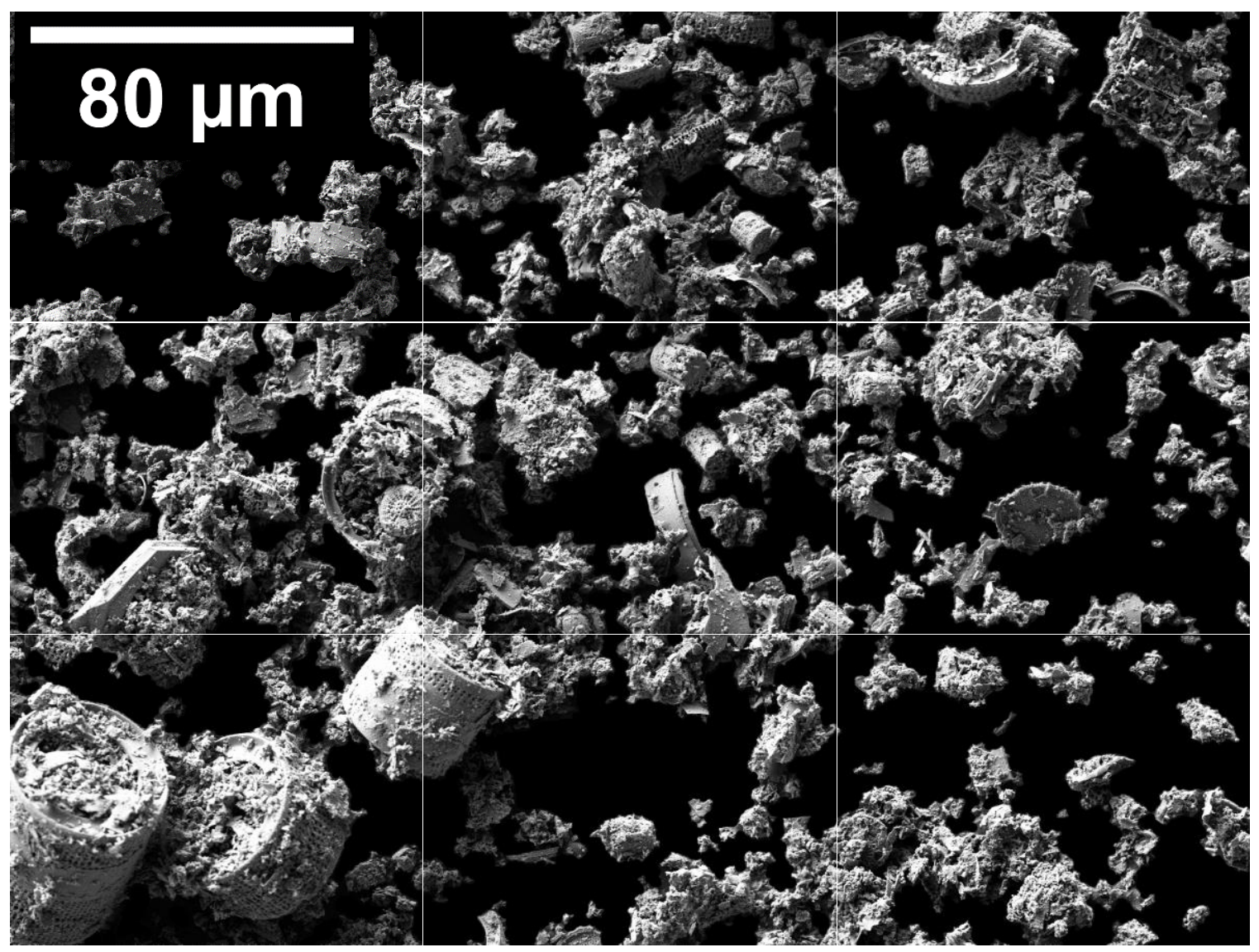

Figure 5-6. Mosaic II, $3 \times 3$ image mosaic of SEM images with all particles on a black background from specimen 01 from sample PC1N13 from the Pinecone Way, Chiloquin field site. Images were captured at a magnification of 3000, and the scale bar of $80 \mu \mathrm{m}$ on the top right image applies for all images. 


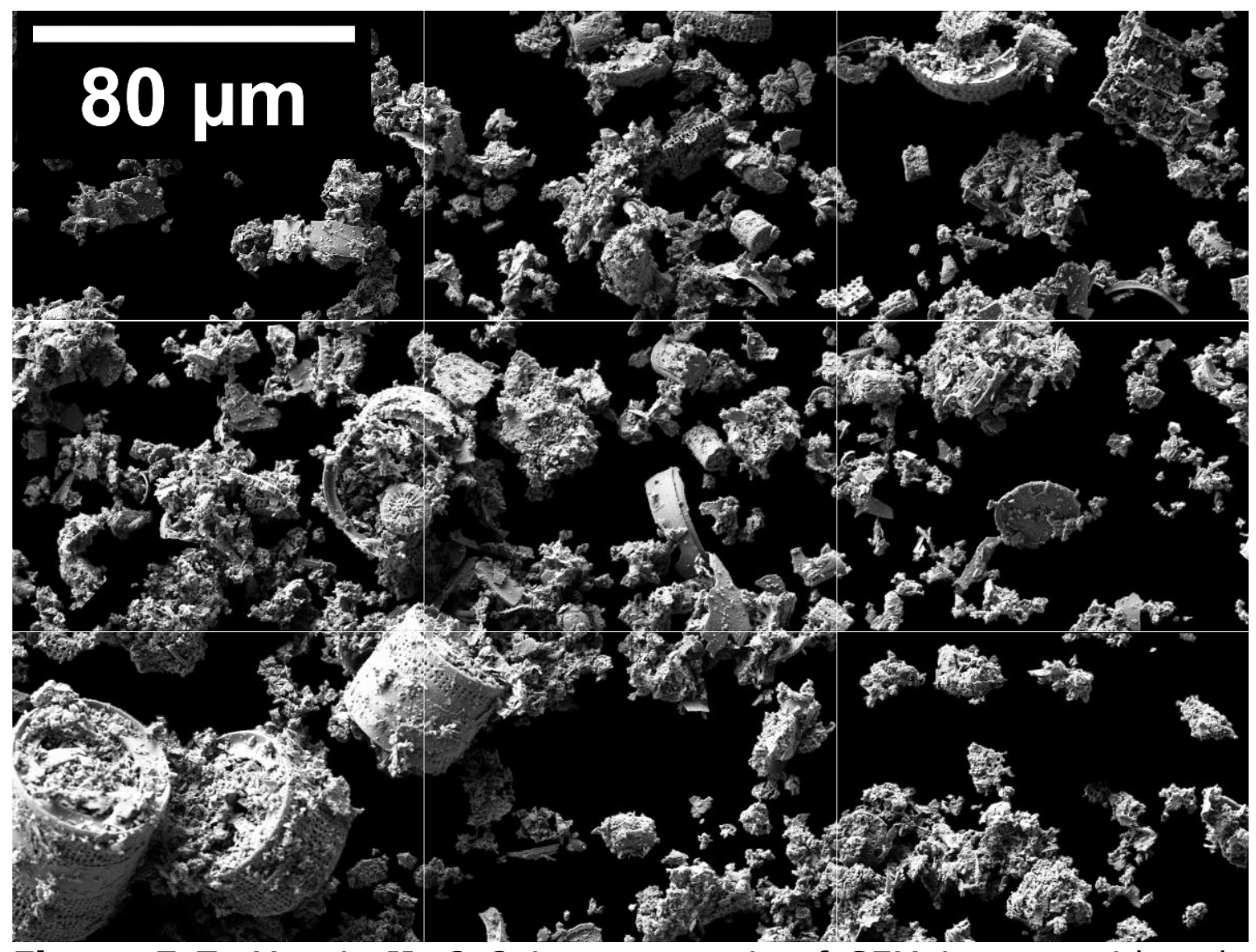

Figure 5-7. Mosaic II, $3 \times 3$ image mosaic of SEM images with only diatom particles on a black background from specimen 01 from sample PC1N13 from the Pinecone Way, Chiloquin field site. Images were captured at a magnification of 3000 , and the scale bar of $80 \mu \mathrm{m}$ on the top right image applies for all images. 


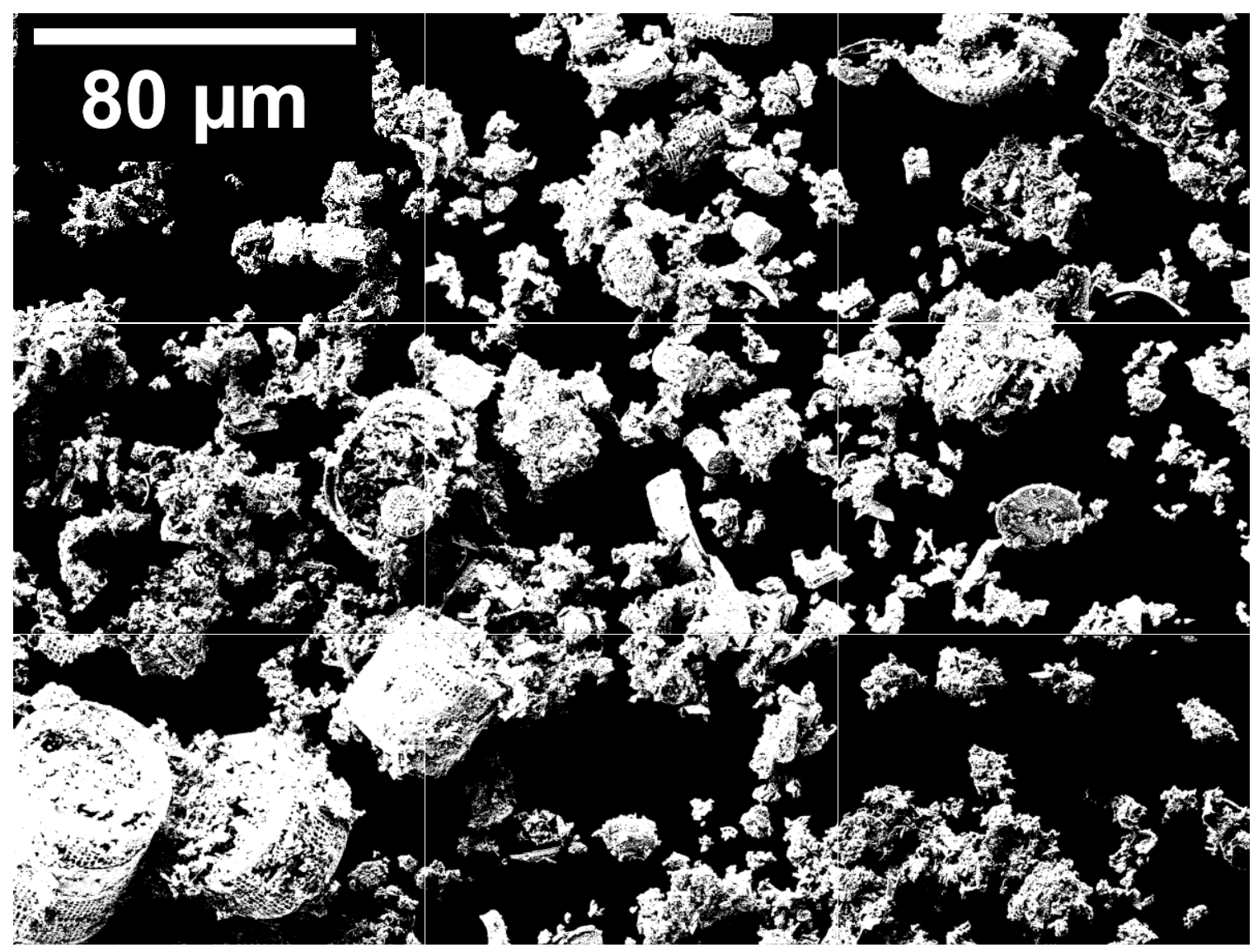

Figure 5-8. Mosaic II, $3 \times 3$ image mosaic of processed SEM images with only diatom particles on a black background from specimen 01 from sample PC1N13 from the Pinecone Way, Chiloquin field site. Images were captured at a magnification of 3000, and the scale bar of $80 \mu \mathrm{m}$ on the top right image applies for all images. 


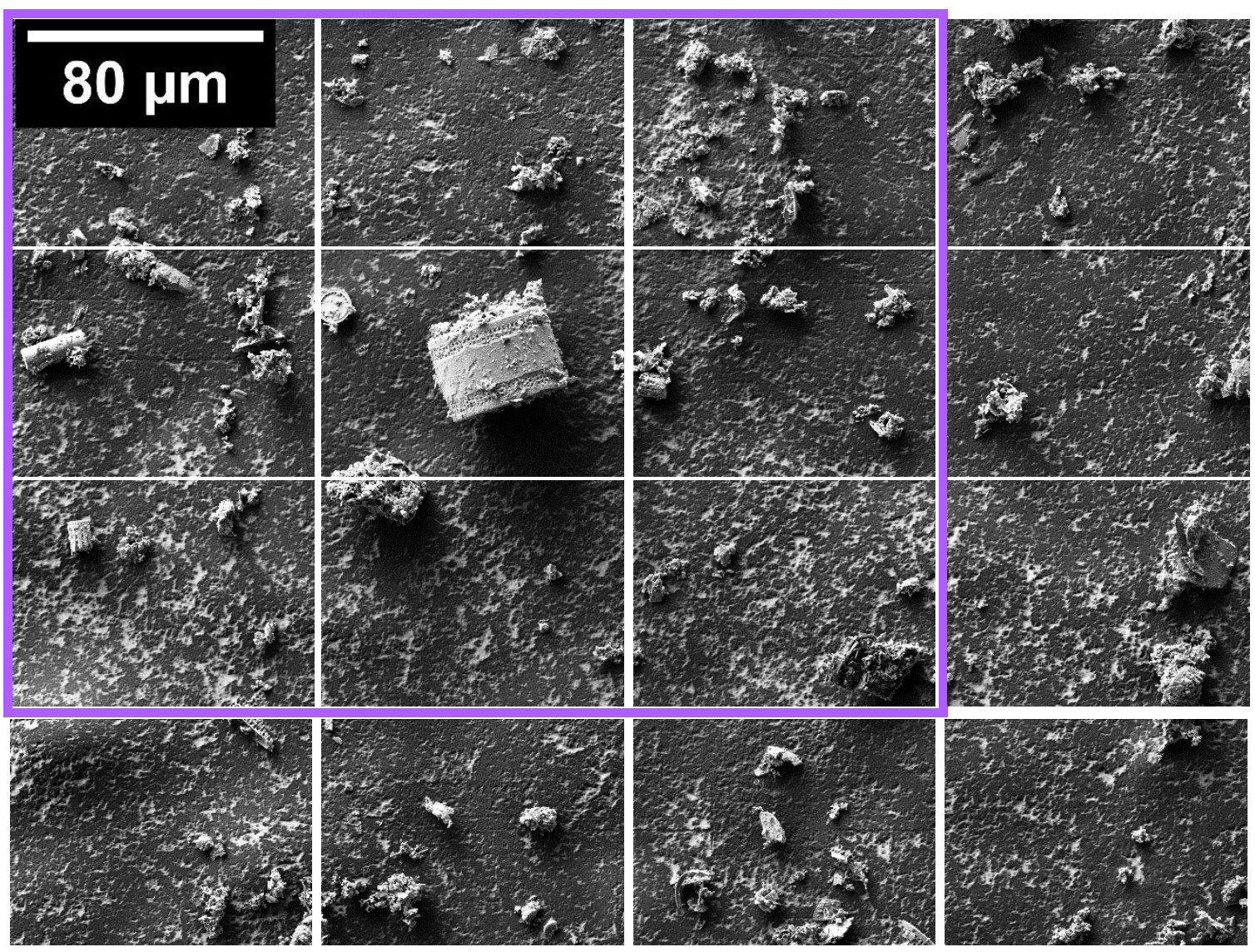

Figure 5-9. 4x4 image mosaic of original SEM images from specimen 01 from sample PC1N13 from Pinecone Way, Chiloquin field site. The purple box illustrates the chosen $3 \times 3$ image mosaic chosen for analysis, Mosaic III. Images were captured at a magnification of 3000, and the scale bar of $80 \mu \mathrm{m}$ on the top right image applies for all images. 


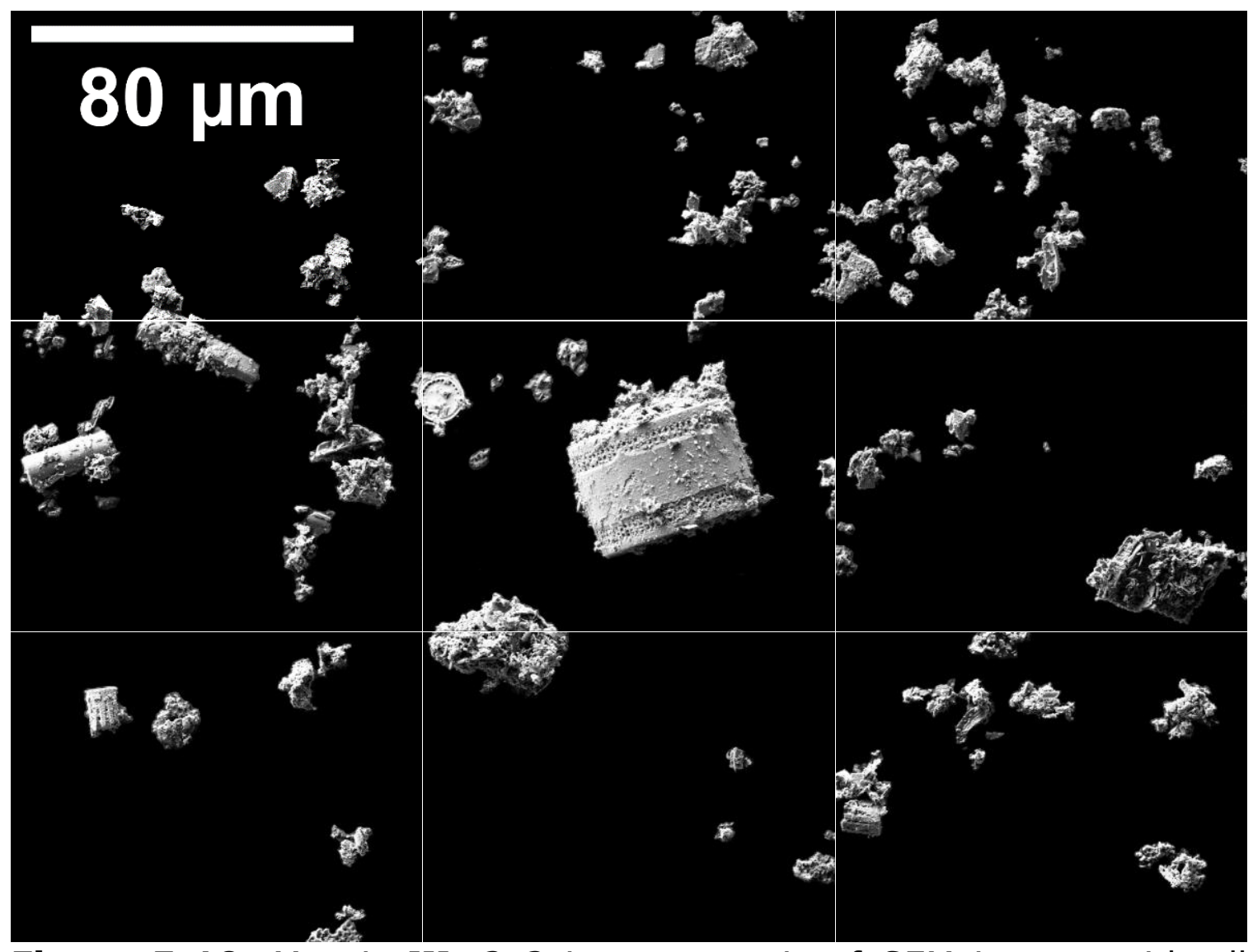

Figure 5-10. Mosaic III, $3 \times 3$ image mosaic of SEM images with all particles on a black background from specimen 01 from sample PC1N13 from the Pinecone Way, Chiloquin field site. Images were captured at a magnification of 3000, and the scale bar of $80 \mu \mathrm{m}$ on the top right image applies for all images. 


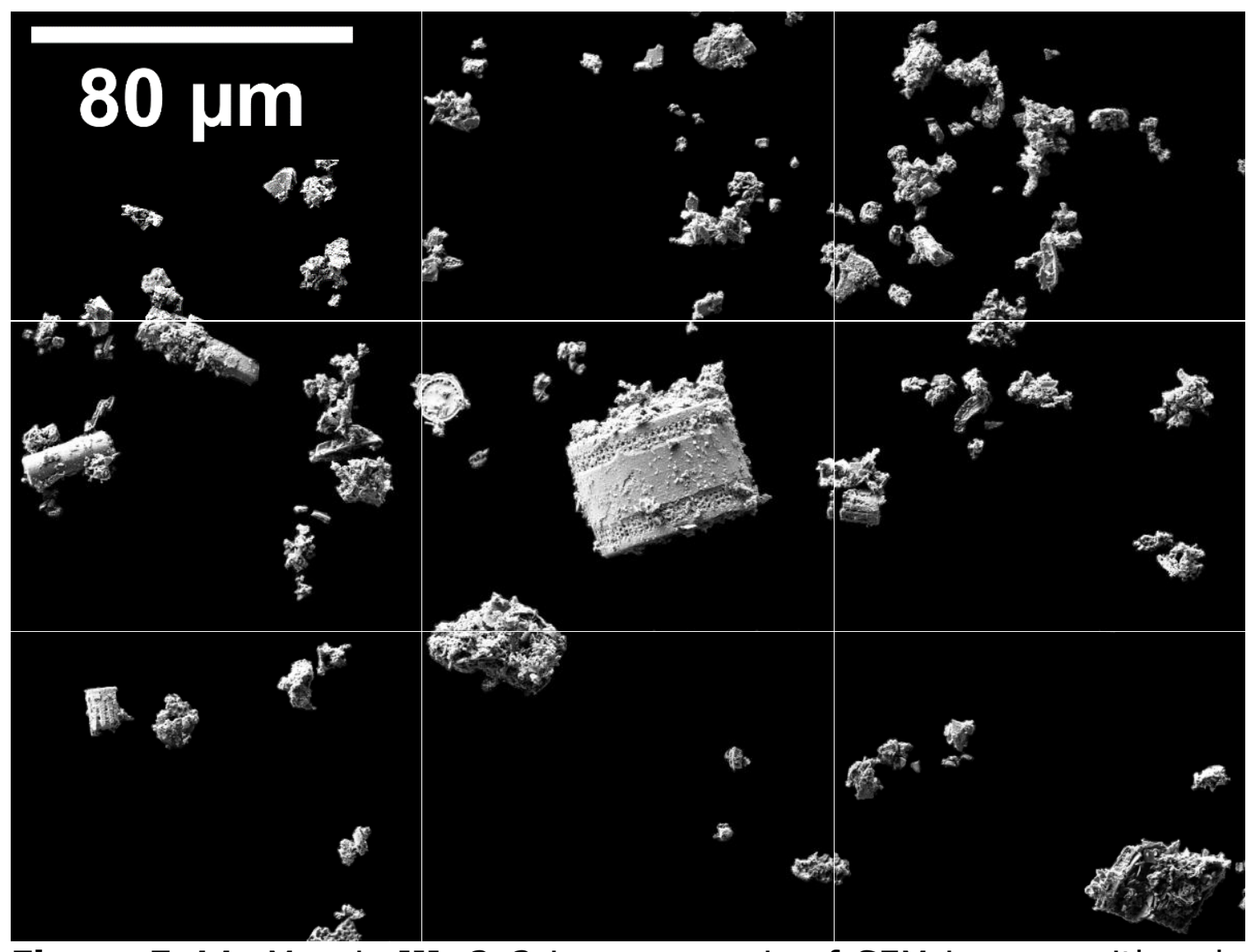

Figure 5-11. Mosaic III, $3 \times 3$ image mosaic of SEM images with only diatom particles on a black background from specimen 01 from sample PC1N13 from the Pinecone Way, Chiloquin field site. Images were captured at a magnification of 3000 , and the scale bar of $80 \mu \mathrm{m}$ on the top right image applies for all images. 


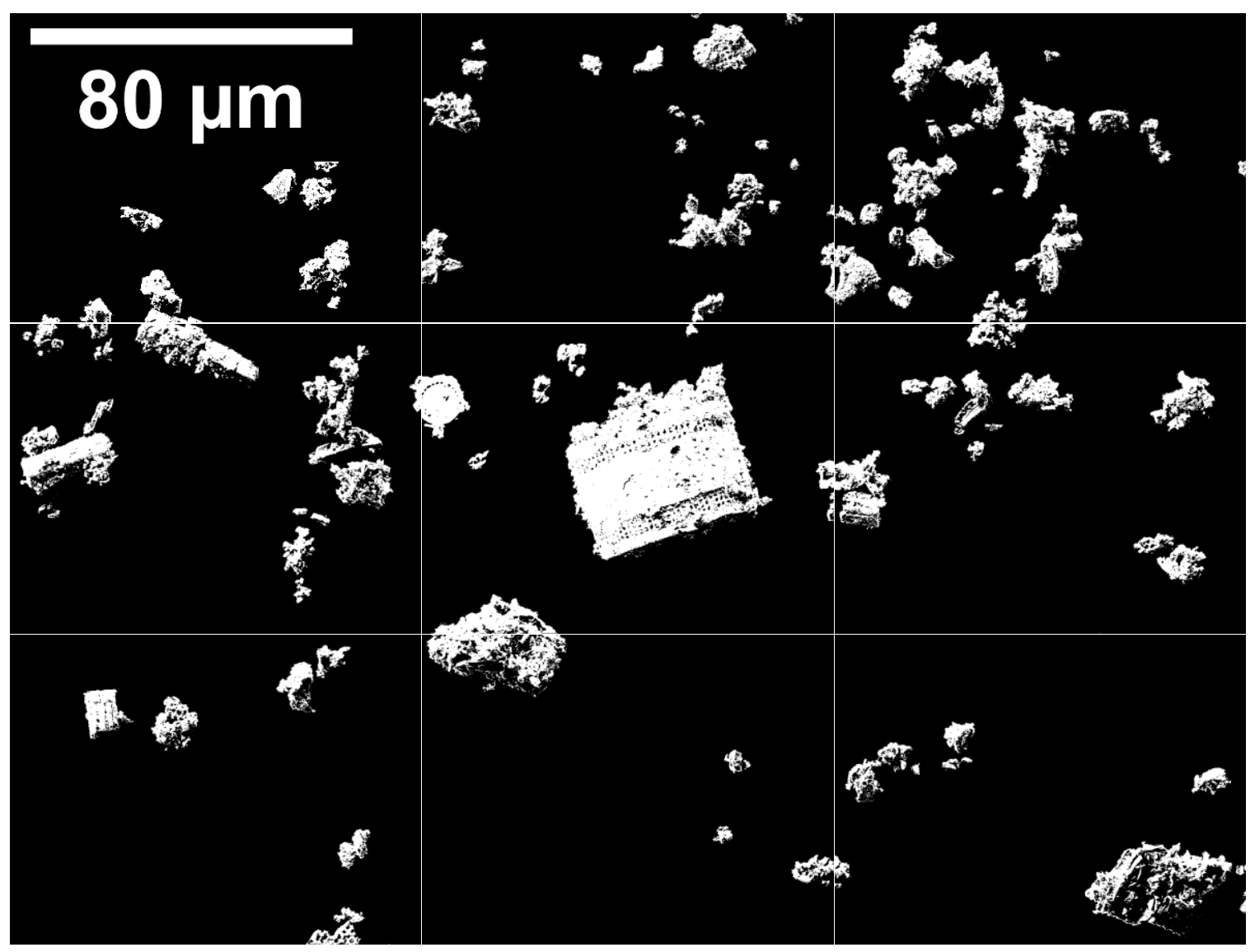

Figure 5-12. Mosaic III, $3 \times 3$ image mosaic of processed SEM images with only diatom particles on a black background from specimen 01 from sample PC1N13 from the Pinecone Way, Chiloquin field site. Images were captured at a magnification of 3000, and the scale bar of $80 \mu \mathrm{m}$ on the top right image applies for all images. 


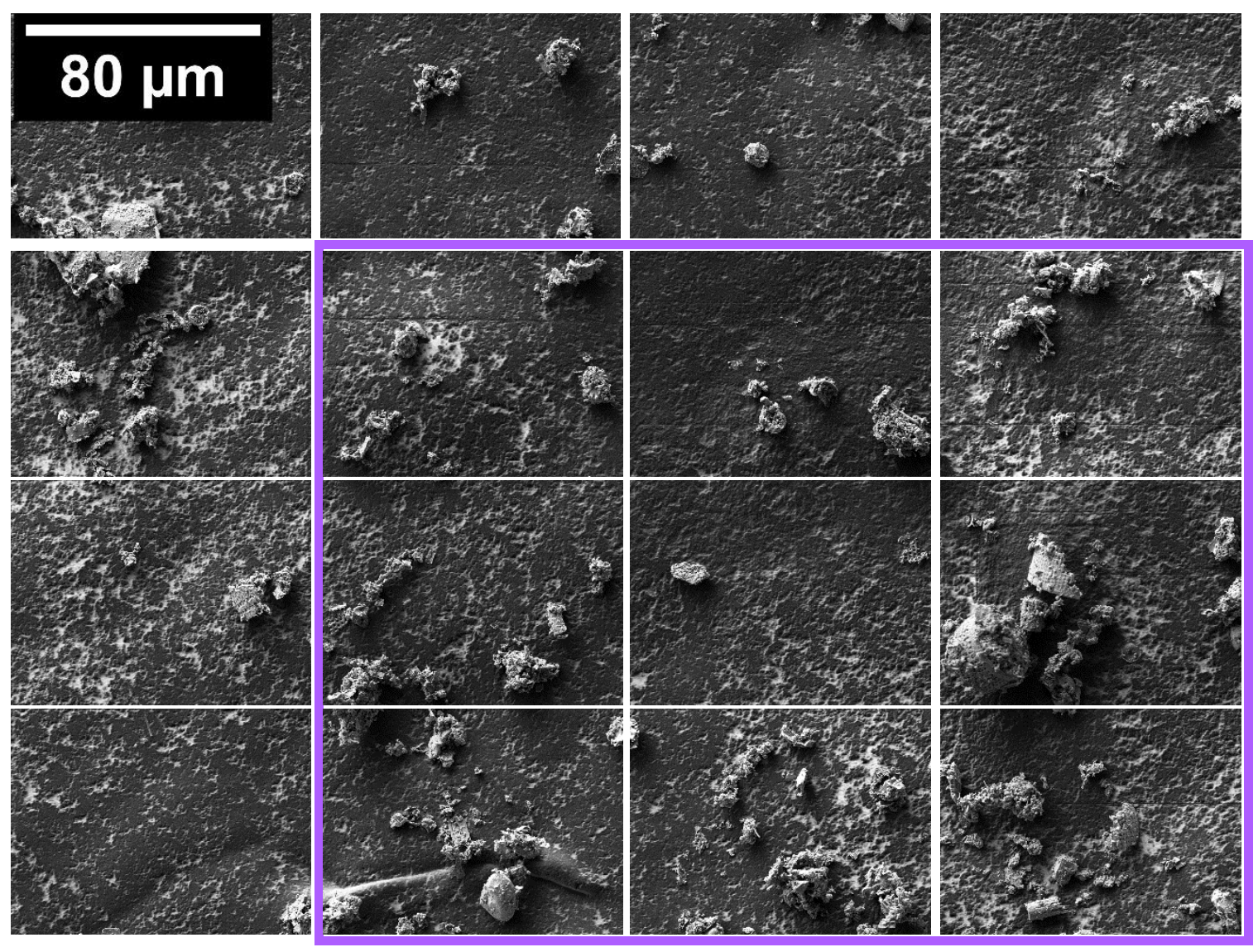

Figure 5-13. $4 \times 4$ image mosaic of original SEM images from specimen 01 from sample PC1N13 from Pinecone Way, Chiloquin field site. The purple box illustrates the chosen $3 \times 3$ image mosaic chosen for analysis, Mosaic IV. Images were captured at a magnification of 3000, and the scale bar of $80 \mu \mathrm{m}$ on the top right image applies for all images. 


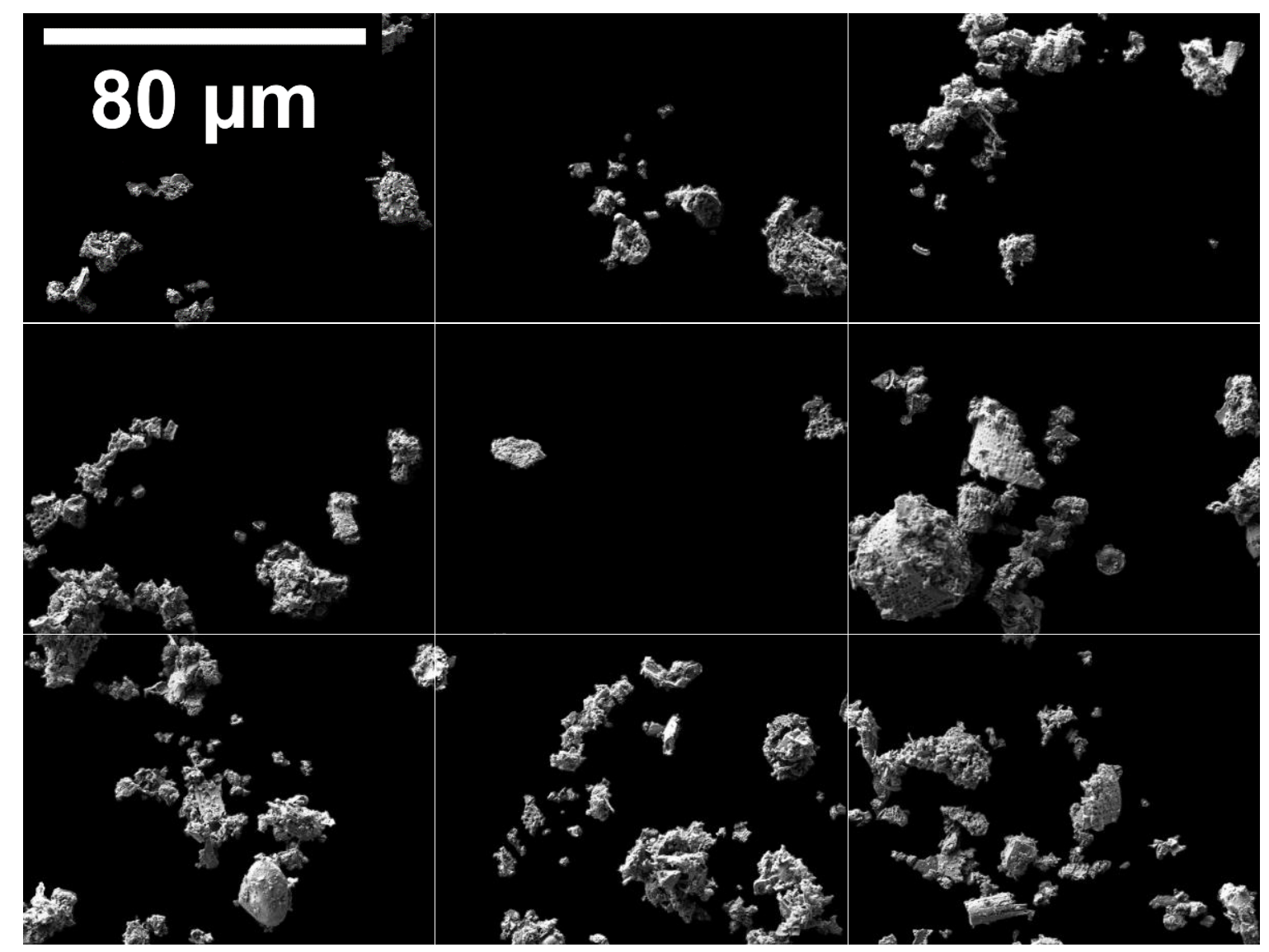

Figure 5-14. Mosaic IV, $3 \times 3$ image mosaic of SEM images with all particles on a black background from specimen 01 from sample PC1N13 from the Pinecone Way, Chiloquin field site. Images were captured at a magnification of 3000 , and the scale bar of $80 \mu \mathrm{m}$ on the top right image applies for all images. 


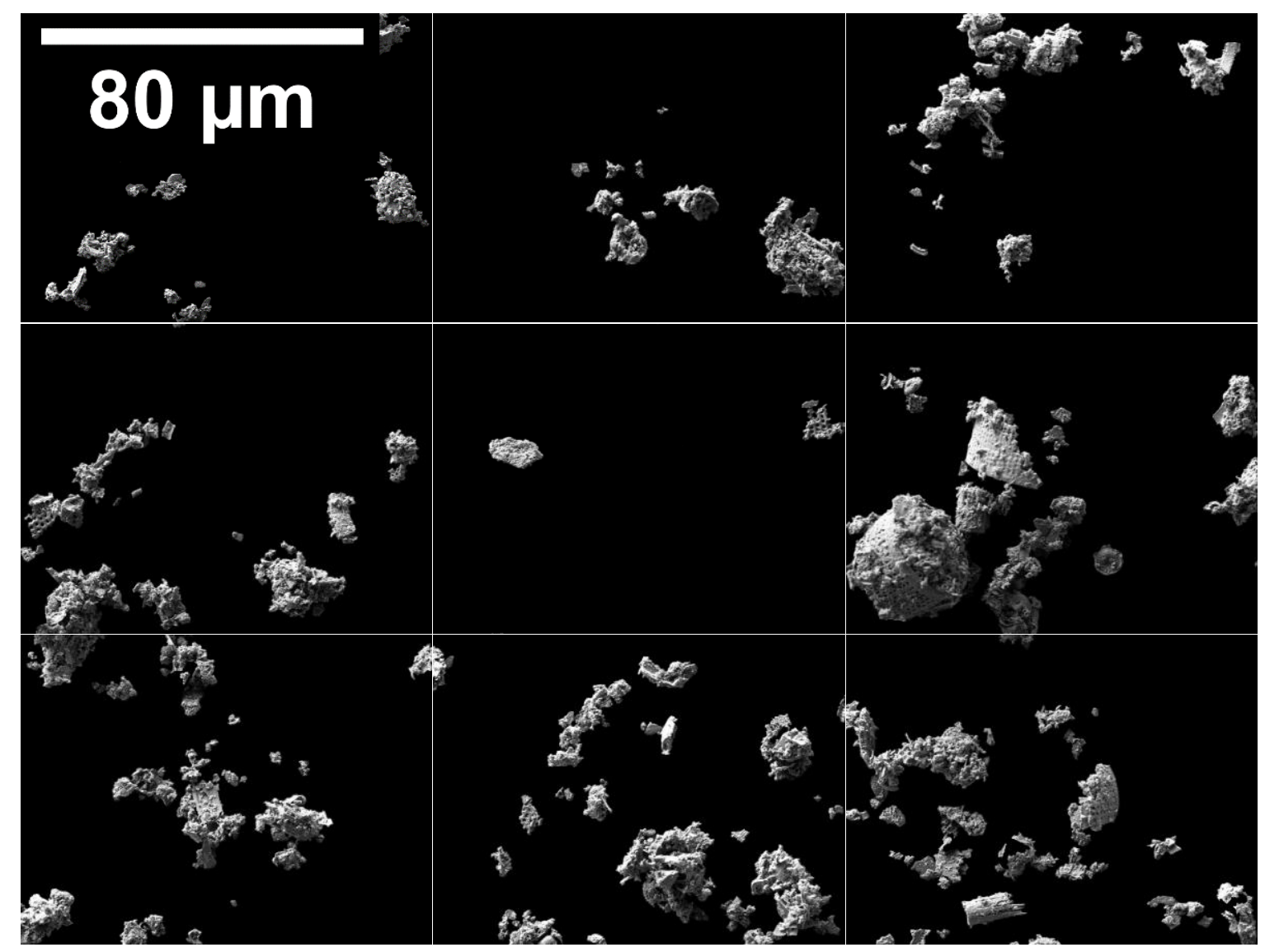

Figure 5-15. Mosaic IV, $3 \times 3$ image mosaic of SEM images with only diatom particles on a black background from specimen 01 from sample PC1N13 from the Pinecone Way, Chiloquin field site. Images were captured at a magnification of 3000 , and the scale bar of $80 \mu \mathrm{m}$ on the top right image applies for all images. 


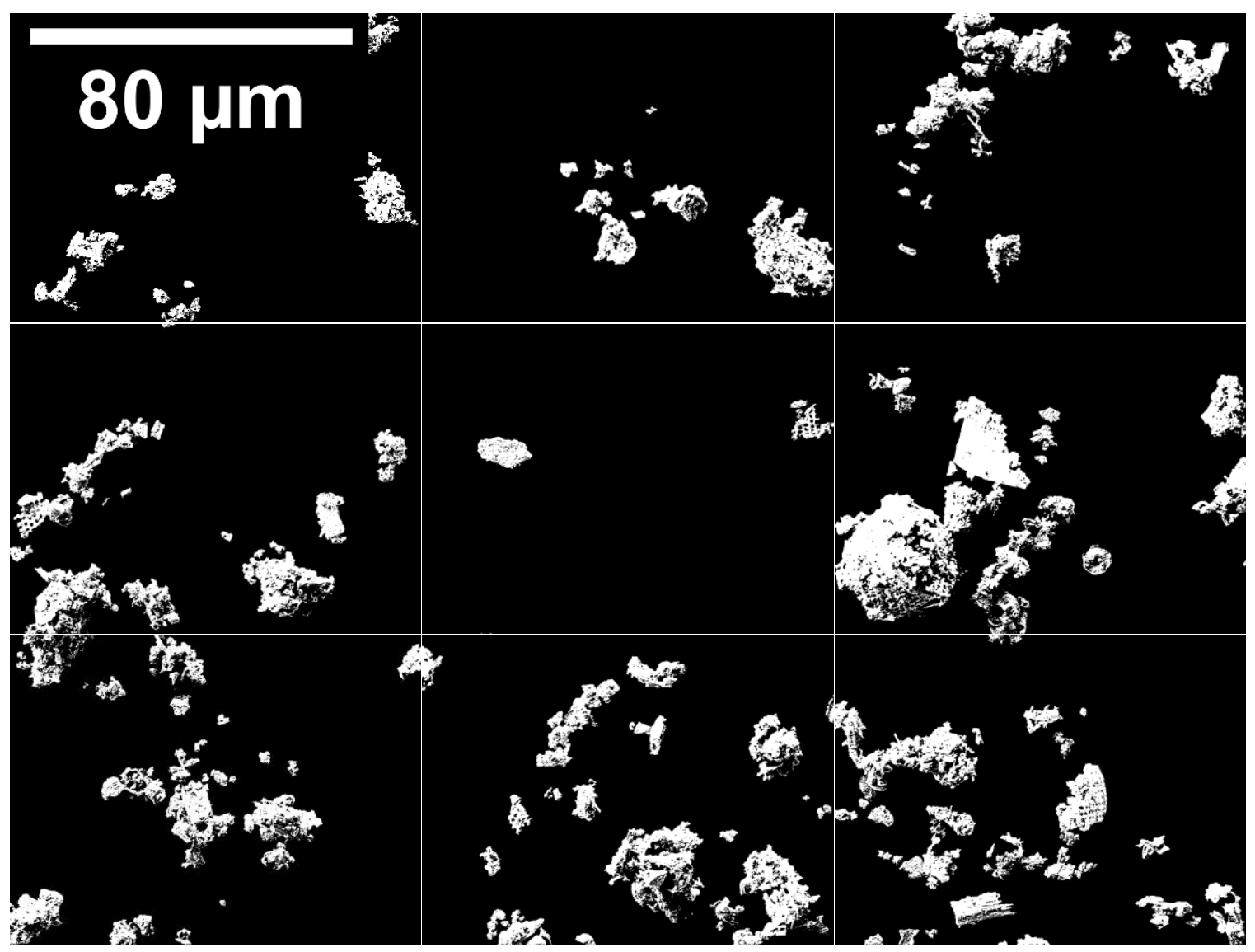

Figure 5-16. Mosaic IV, $3 \times 3$ image mosaic of processed SEM images with only diatom particles on a black background from specimen 01 from sample PC1N13 from the Pinecone Way, Chiloquin field site. Images were captured at a magnification of 3000, and the scale bar of $80 \mu \mathrm{m}$ on the top right image applies for all images. 


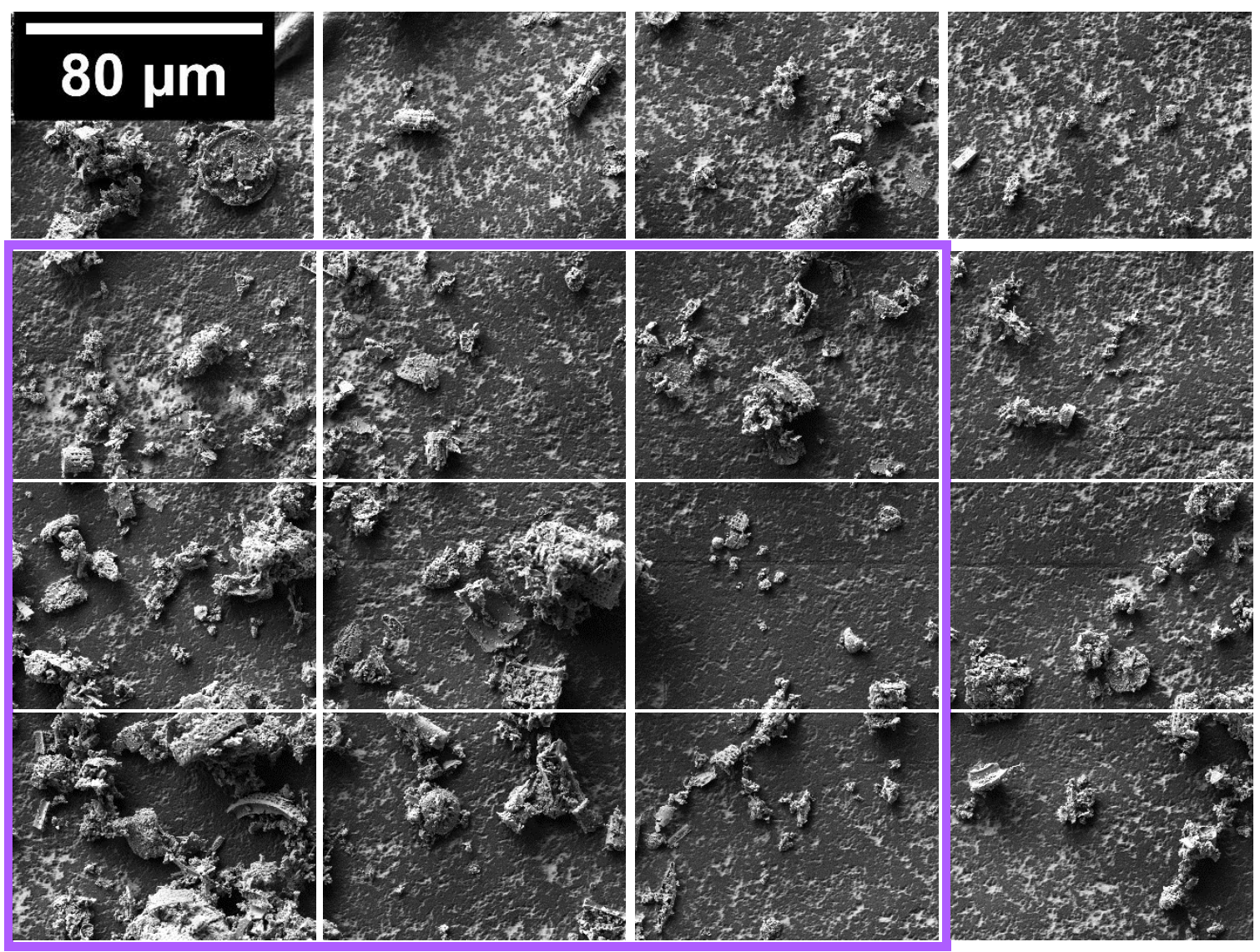

Figure 5-17. $4 \times 4$ image mosaic of original SEM images from specimen 02 from sample PC1N13 from Pinecone Way, Chiloquin field site. The purple box illustrates the chosen $3 \times 3$ image mosaic chosen for analysis, Mosaic I. Images were captured at a magnification of 3000, and the scale bar of $80 \mu \mathrm{m}$ on the top right image applies for all images. 


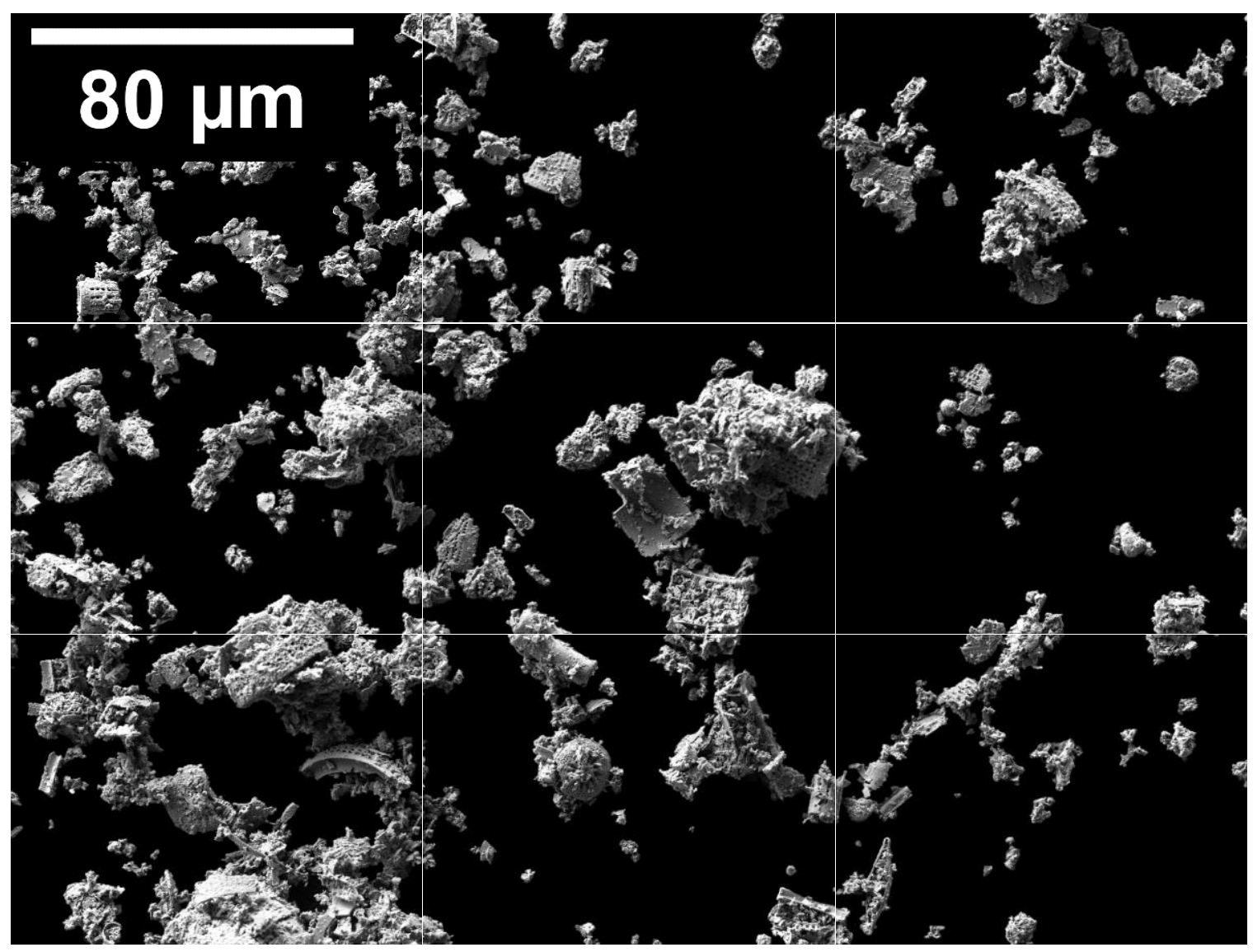

Figure 5-18. Mosaic I, $3 \times 3$ image mosaic of SEM images with all particles on a black background from specimen 02 from sample PC1N13 from the Pinecone Way, Chiloquin field site. Images were captured at a magnification of 3000 , and the scale bar of $80 \mu \mathrm{m}$ on the top right image applies for all images. 


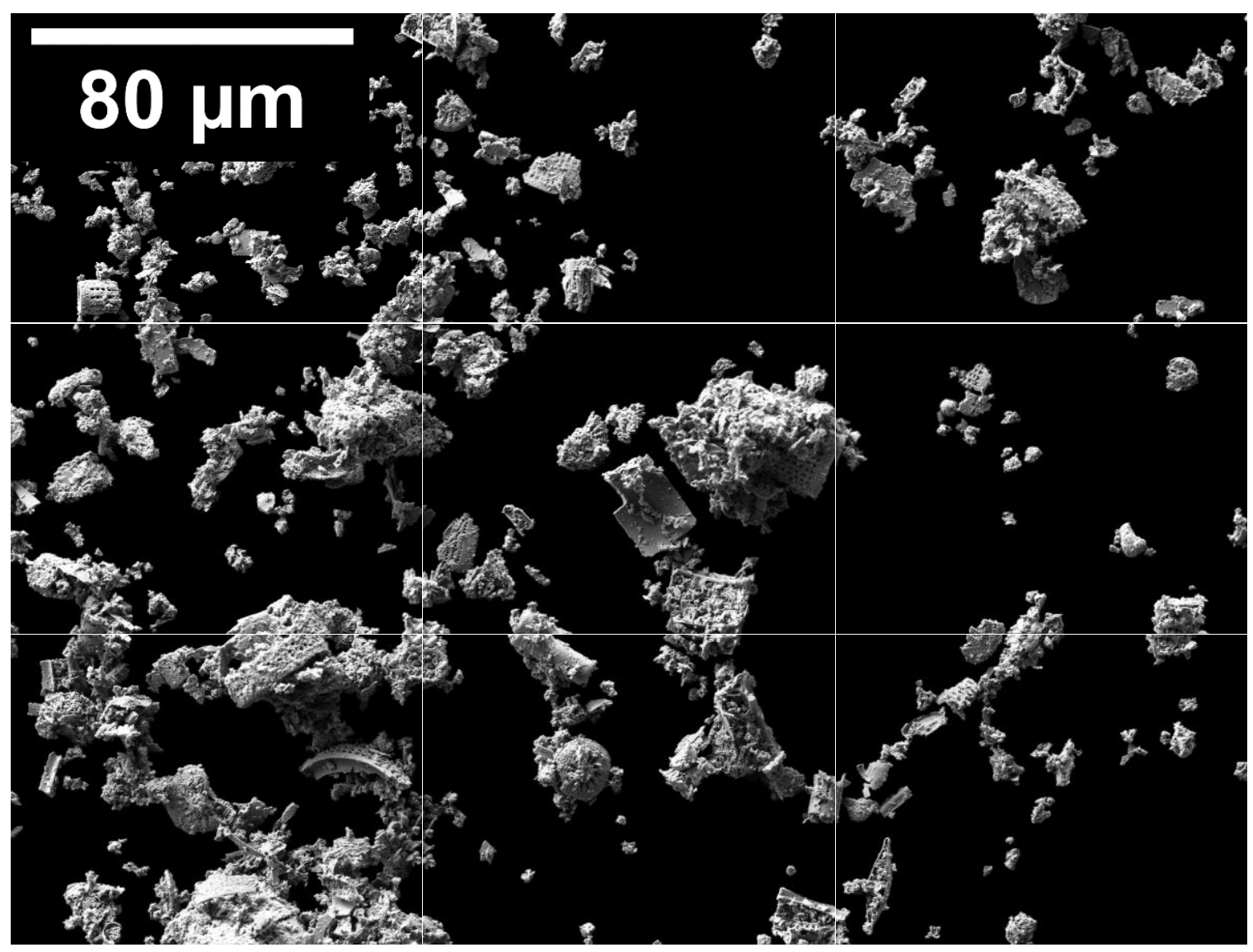

Figure 5-19. Mosaic I, $3 \times 3$ image mosaic of SEM images with only diatom particles on a black background from specimen 02 from sample PC1N13 from the Pinecone Way, Chiloquin field site. Images were captured at a magnification of 3000 , and the scale bar of $80 \mu \mathrm{m}$ on the top right image applies for all images. 


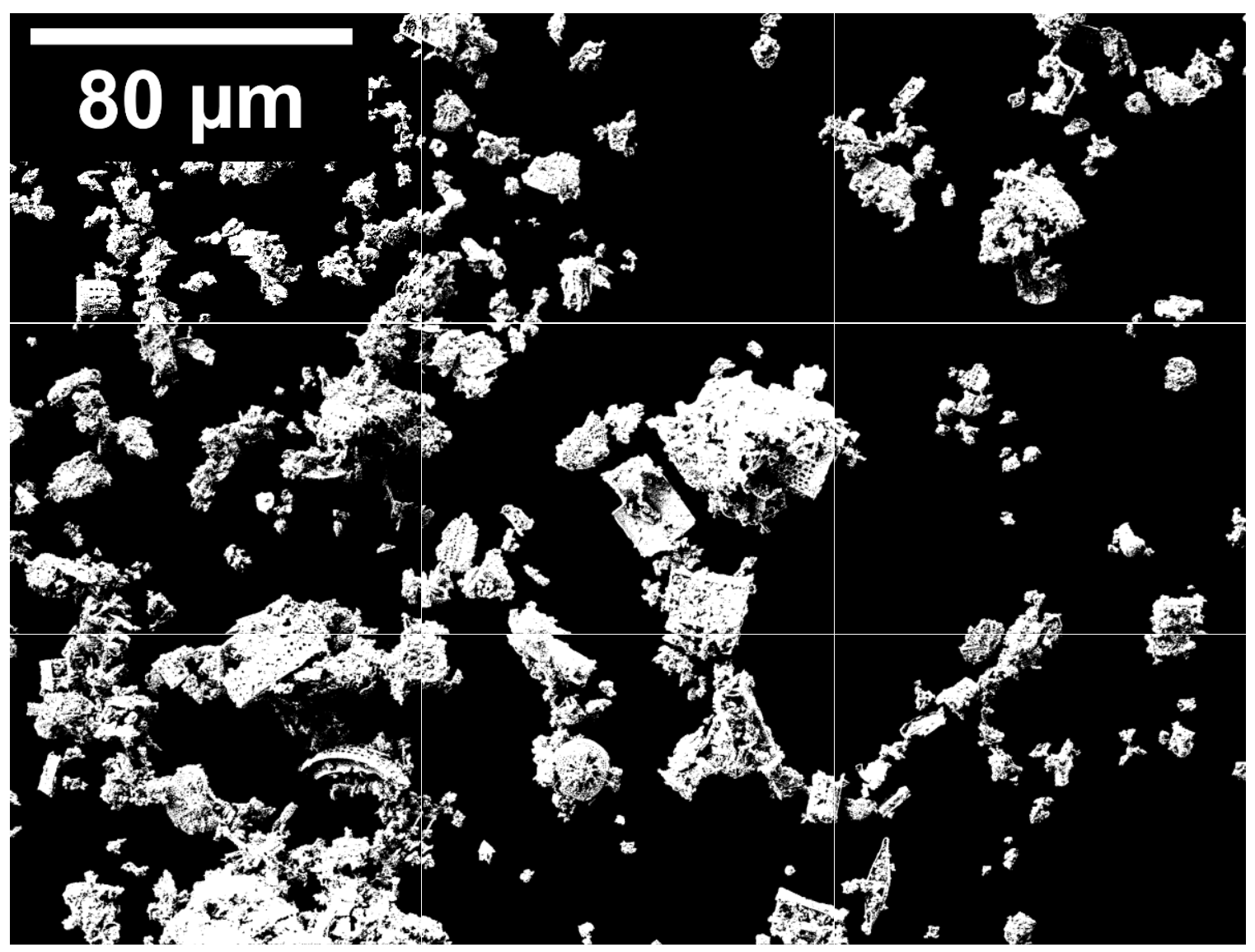

Figure 5-20. Mosaic I, $3 \times 3$ image mosaic of processed SEM images with only diatom particles on a black background from specimen 02 from sample PC1N13 from the Pinecone Way, Chiloquin field site. Images were captured at a magnification of 3000, and the scale bar of $80 \mu \mathrm{m}$ on the top right image applies for all images. 


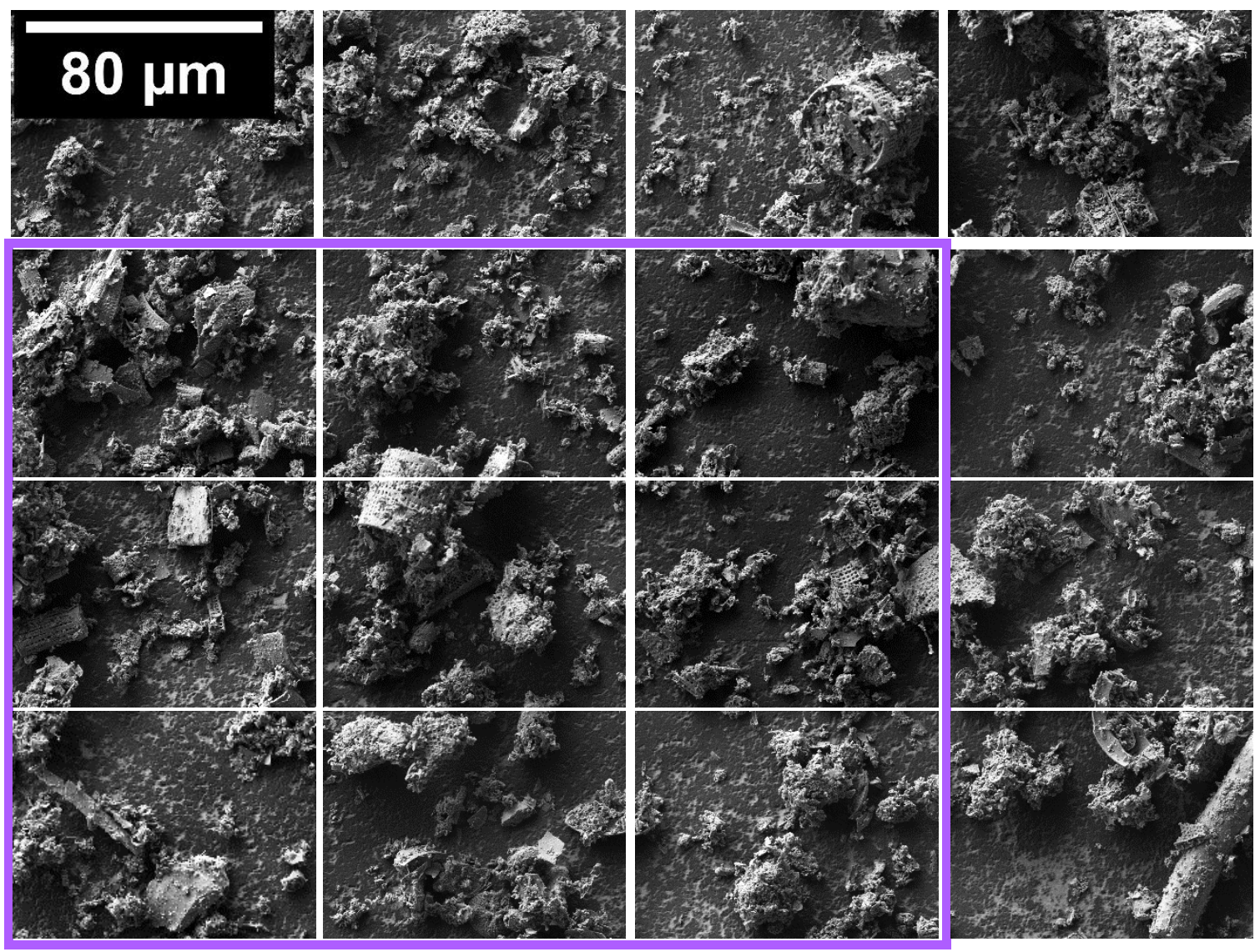

Figure 5-21. $4 \times 4$ image mosaic of original SEM images from specimen 02 from sample PC1N13 from Pinecone Way, Chiloquin field site. The purple box illustrates the chosen $3 \times 3$ image mosaic chosen for analysis, Mosaic II. Images were captured at a magnification of 3000, and the scale bar of $80 \mu \mathrm{m}$ on the top right image applies for all images. 


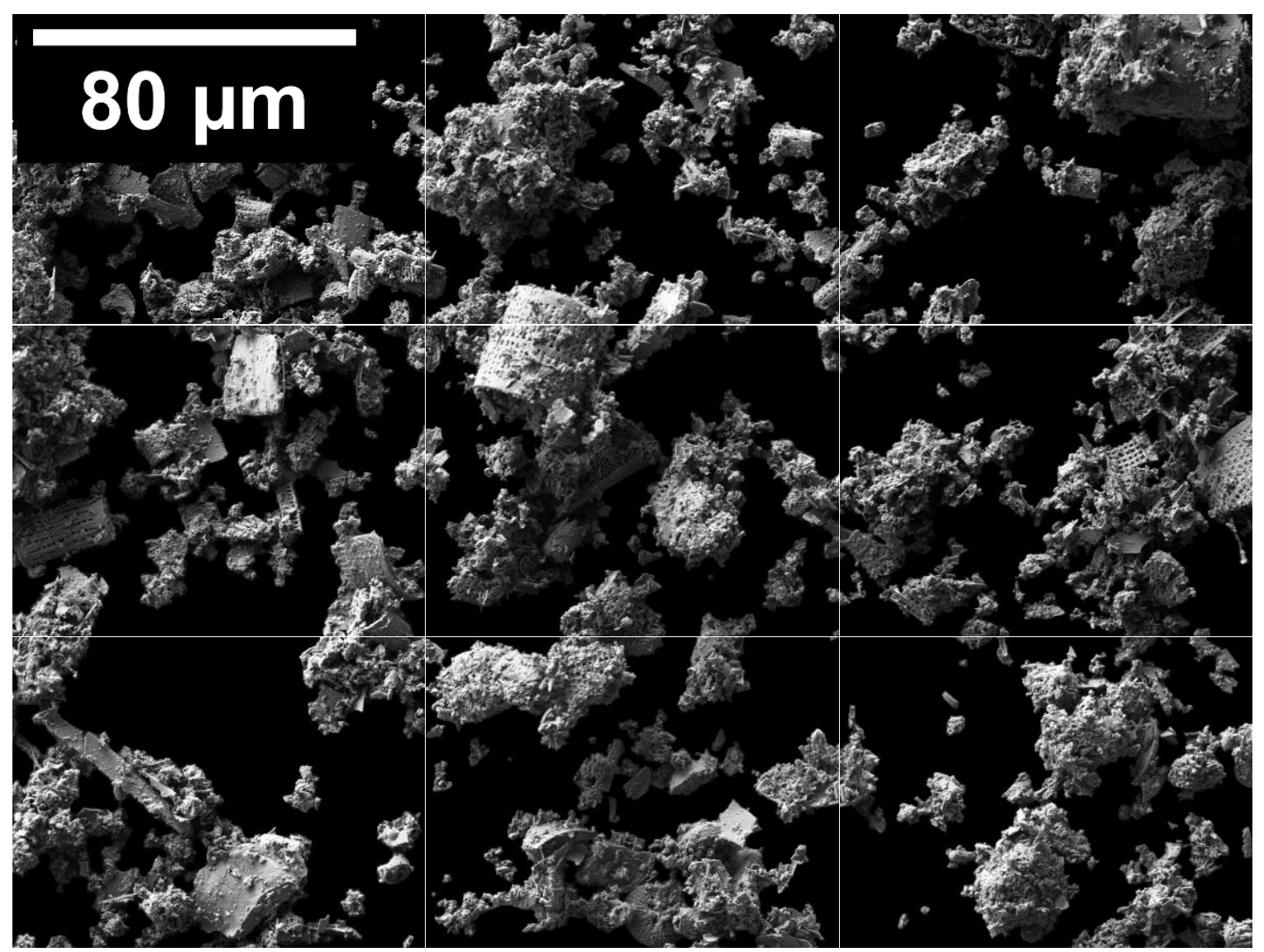

Figure 5-22. Mosaic II, $3 \times 3$ image mosaic of SEM images with all particles on a black background from specimen 02 from sample PC1N13 from the Pinecone Way, Chiloquin field site. Images were captured at a magnification of 3000 , and the scale bar of $80 \mu \mathrm{m}$ on the top right image applies for all images. 


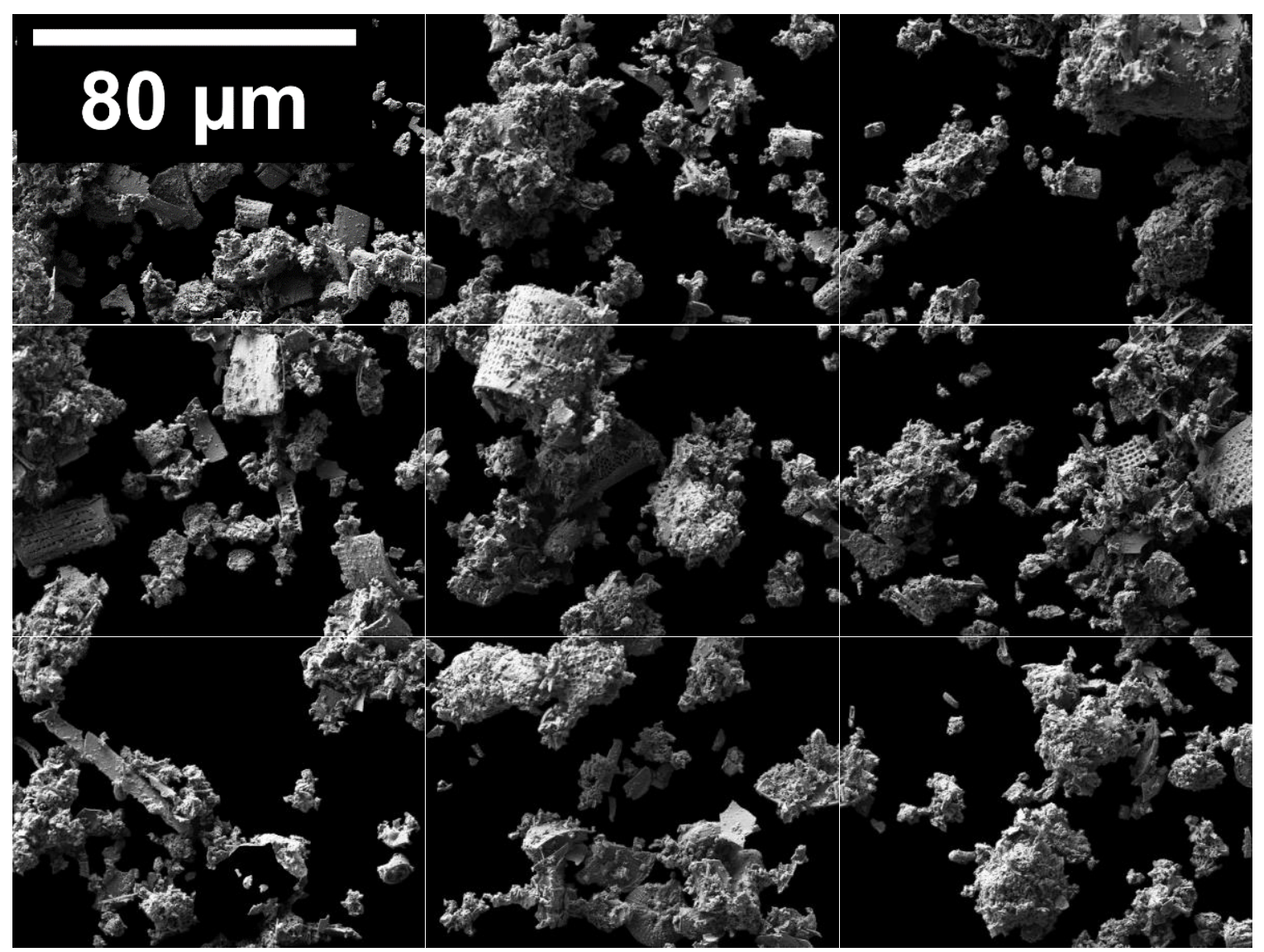

Figure 5-23. Mosaic II, $3 \times 3$ image mosaic of SEM images with only diatom particles on a black background from specimen 02 from sample PC1N13 from the Pinecone Way, Chiloquin field site. Images were captured at a magnification of 3000 , and the scale bar of $80 \mu \mathrm{m}$ on the top right image applies for all images. 


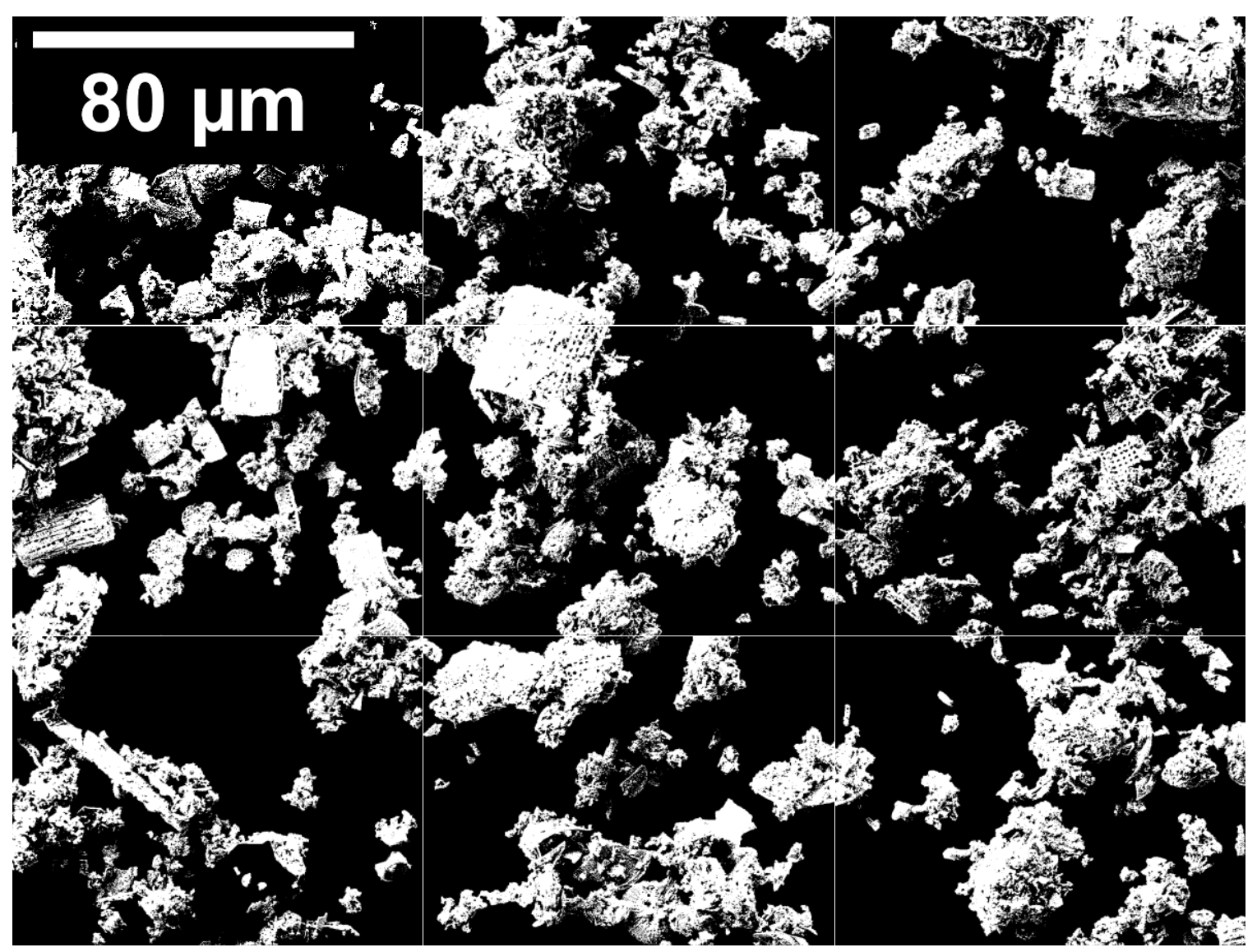

Figure 5-24. Mosaic II, $3 \times 3$ image mosaic of processed SEM images with only diatom particles on a black background from specimen 02 from sample PC1N13 from the Pinecone Way, Chiloquin field site. Images were captured at a magnification of 3000, and the scale bar of $80 \mu \mathrm{m}$ on the top right image applies for all images. 


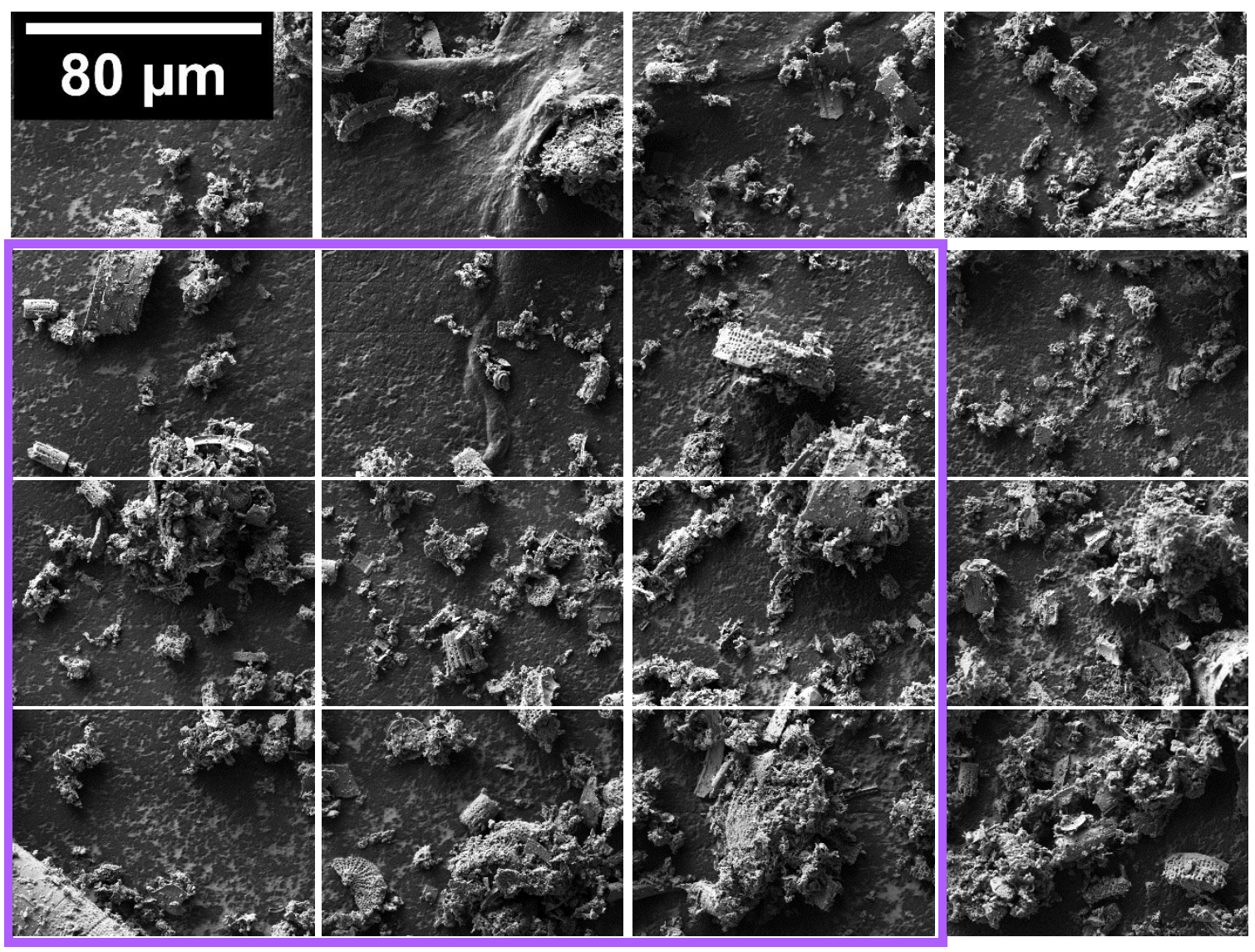

Figure 5-25. 4x4 image mosaic of original SEM images from specimen 02 from sample PC1N13 from Pinecone Way, Chiloquin field site. The purple box illustrates the chosen $3 \times 3$ image mosaic chosen for analysis, Mosaic III. Images were captured at a magnification of 3000, and the scale bar of $80 \mu \mathrm{m}$ on the top right image applies for all images. 


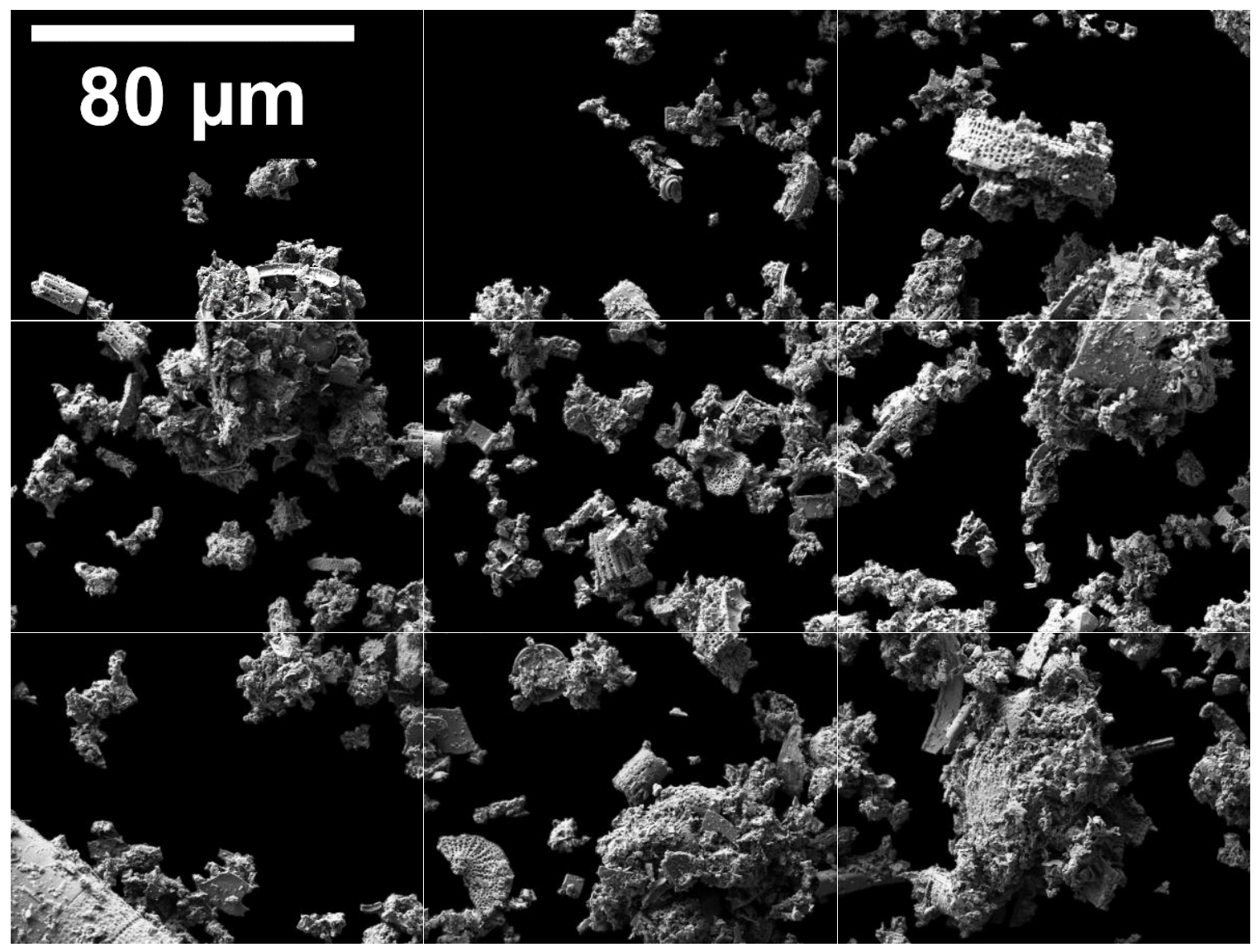

Figure 5-26. Mosaic III, $3 \times 3$ image mosaic of SEM images with all particles on a black background from specimen 02 from sample PC1N13 from the Pinecone Way, Chiloquin field site. Images were captured at a magnification of 3000 , and the scale bar of $80 \mu \mathrm{m}$ on the top right image applies for all images. 


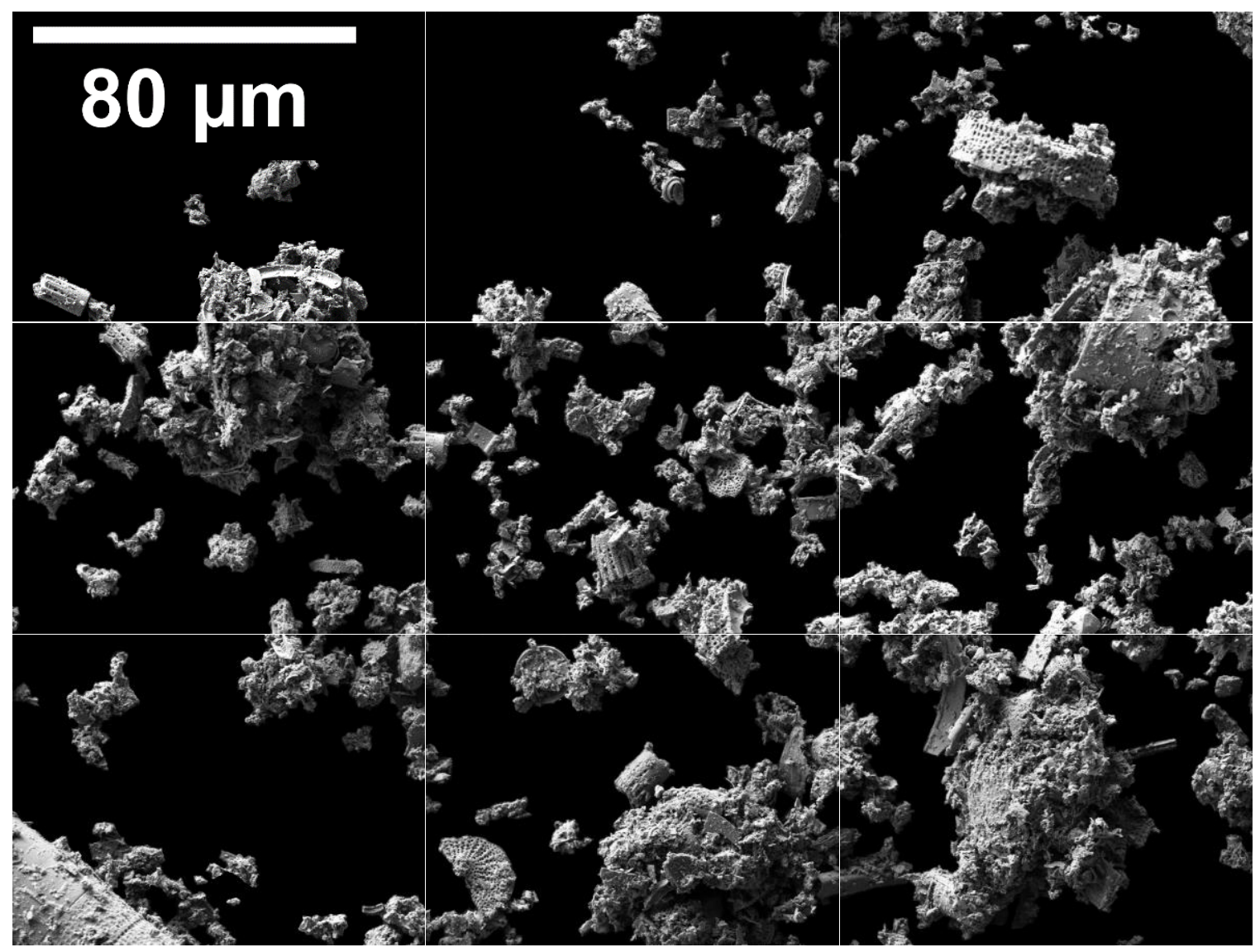

Figure 5-27. Mosaic III, $3 \times 3$ image mosaic of SEM images with only diatom particles on a black background from specimen 02 from sample PC1N13 from the Pinecone Way, Chiloquin field site. Images were captured at a magnification of 3000 , and the scale bar of $80 \mu \mathrm{m}$ on the top right image applies for all images. 


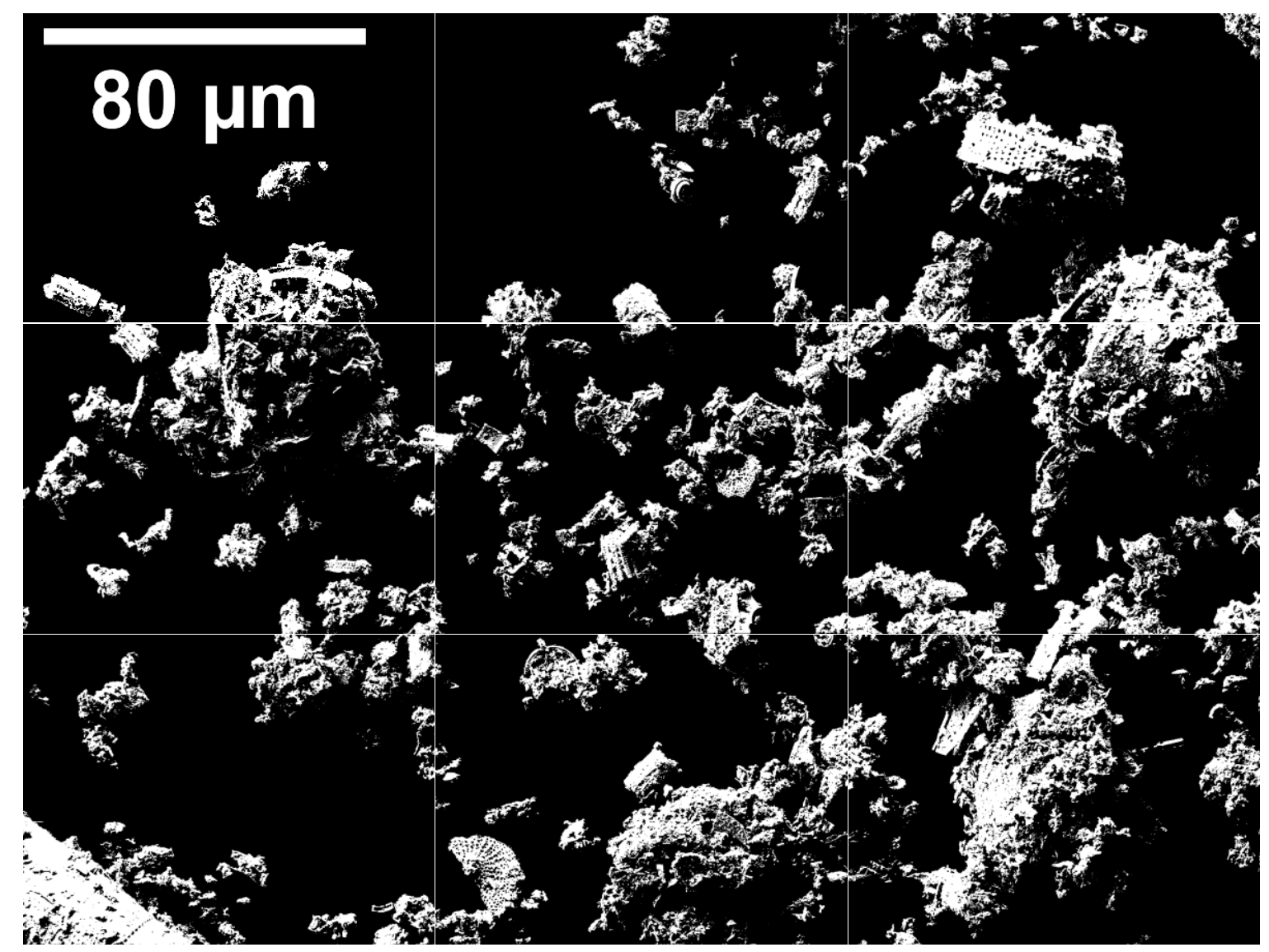

Figure 5-28. Mosaic III, $3 \times 3$ image mosaic of processed SEM images with only diatom particles on a black background from specimen 02 from sample PC1N13 from the Pinecone Way, Chiloquin field site. Images were captured at a magnification of 3000, and the scale bar of $80 \mu \mathrm{m}$ on the top right image applies for all images. 


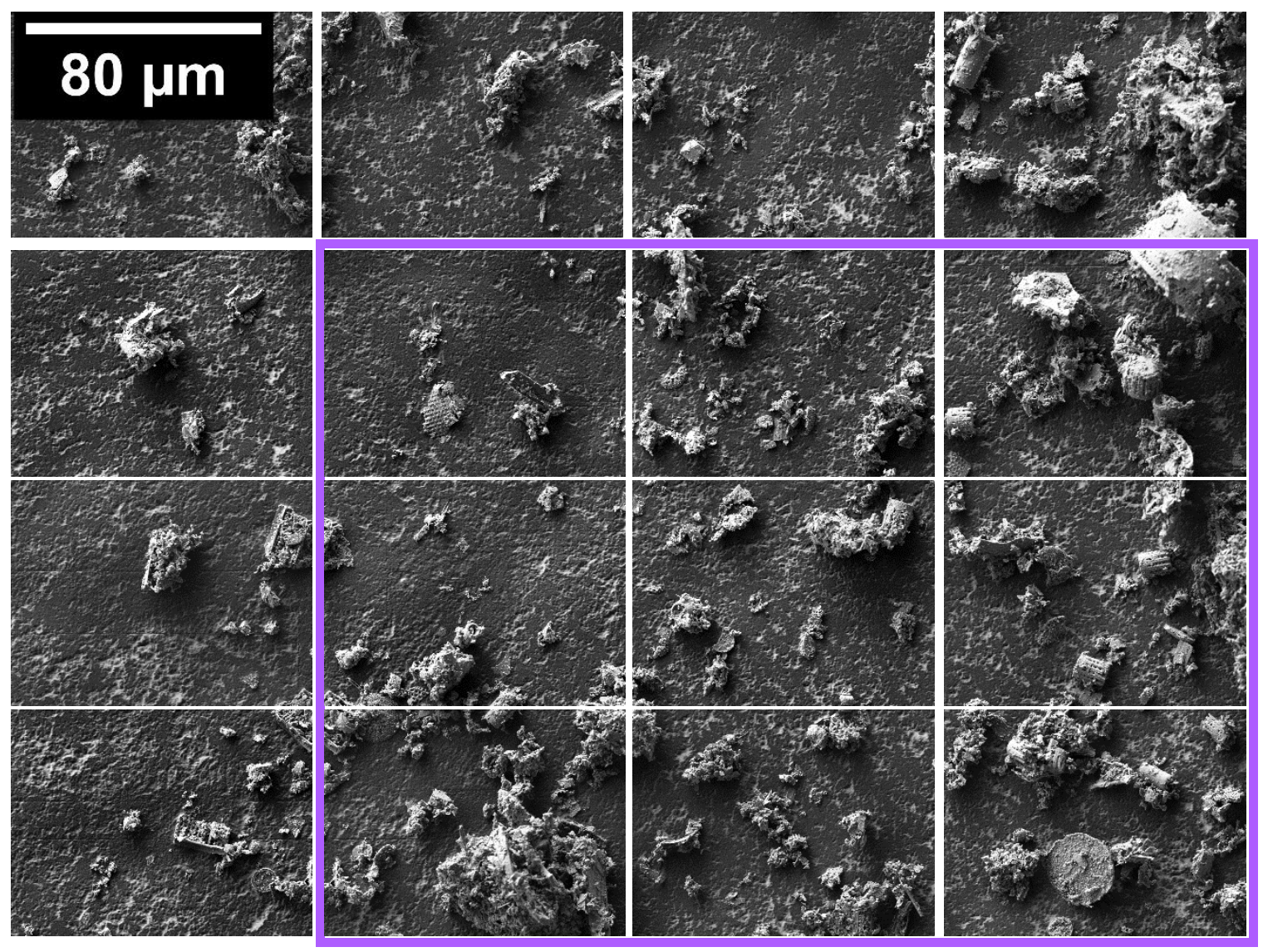

Figure 5-29. $4 \times 4$ image mosaic of original SEM images from specimen 02 from sample PC1N13 from Pinecone Way, Chiloquin field site. The purple box illustrates the chosen $3 \times 3$ image mosaic chosen for analysis, Mosaic IV. Images were captured at a magnification of 3000, and the scale bar of $80 \mu \mathrm{m}$ on the top right image applies for all images. 


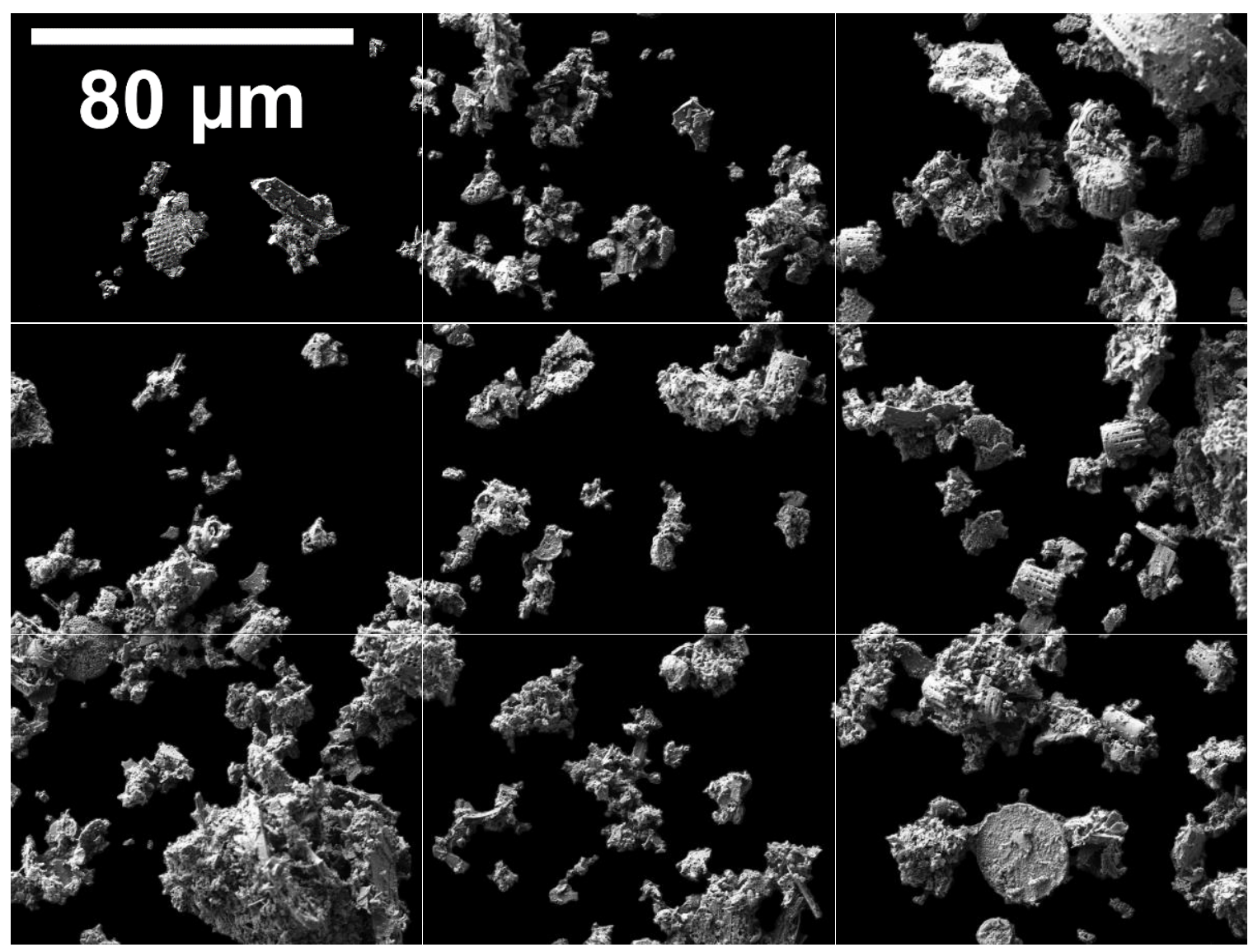

Figure 5-30. Mosaic IV, $3 \times 3$ image mosaic of SEM images with all particles on a black background from specimen 02 from sample PC1N13 from the Pinecone Way, Chiloquin field site. Images were captured at a magnification of 3000 , and the scale bar of $80 \mu \mathrm{m}$ on the top right image applies for all images. 


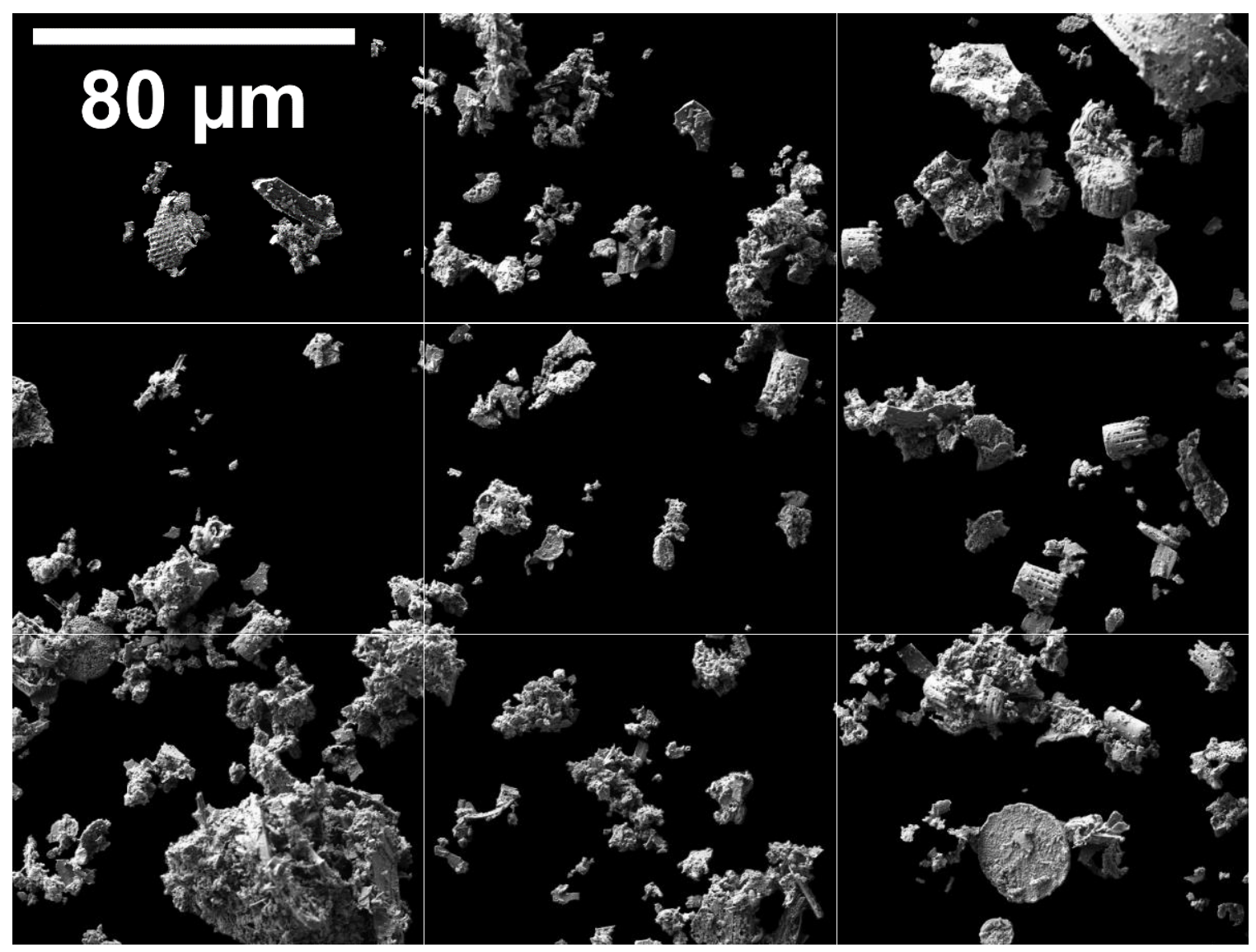

Figure 5-31. Mosaic IV, $3 \times 3$ image mosaic of SEM images with only diatom particles on a black background from specimen 02 from sample PC1N13 from the Pinecone Way, Chiloquin field site. Images were captured at a magnification of 3000 , and the scale bar of $80 \mu \mathrm{m}$ on the top right image applies for all images. 


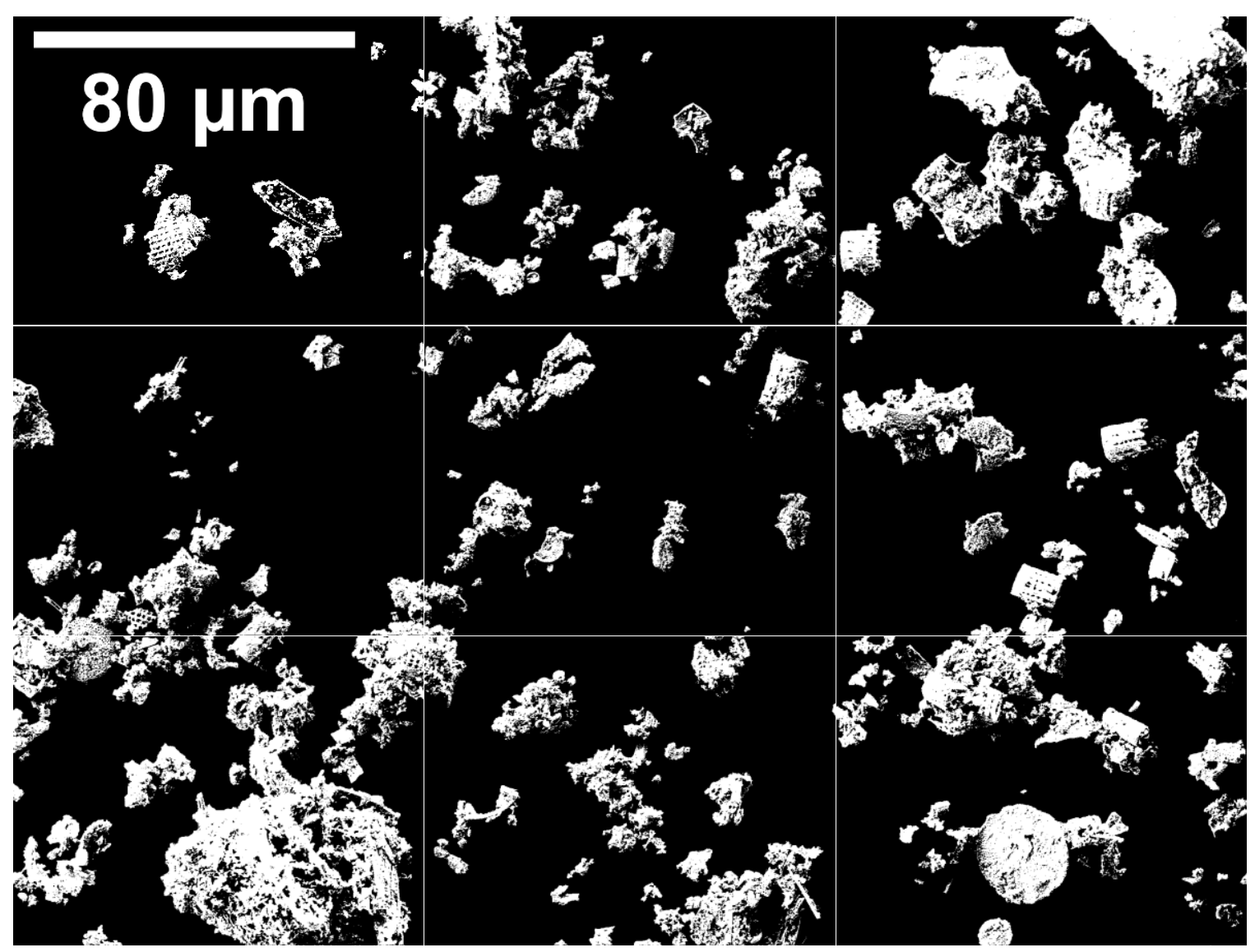

Figure 5-32. Mosaic IV, $3 \times 3$ image mosaic of processed SEM images with only diatom particles on a black background from specimen 02 from sample PC1N13 from the Pinecone Way, Chiloquin field site. Images were captured at a magnification of 3000, and the scale bar of $80 \mu \mathrm{m}$ on the top right image applies for all images. 


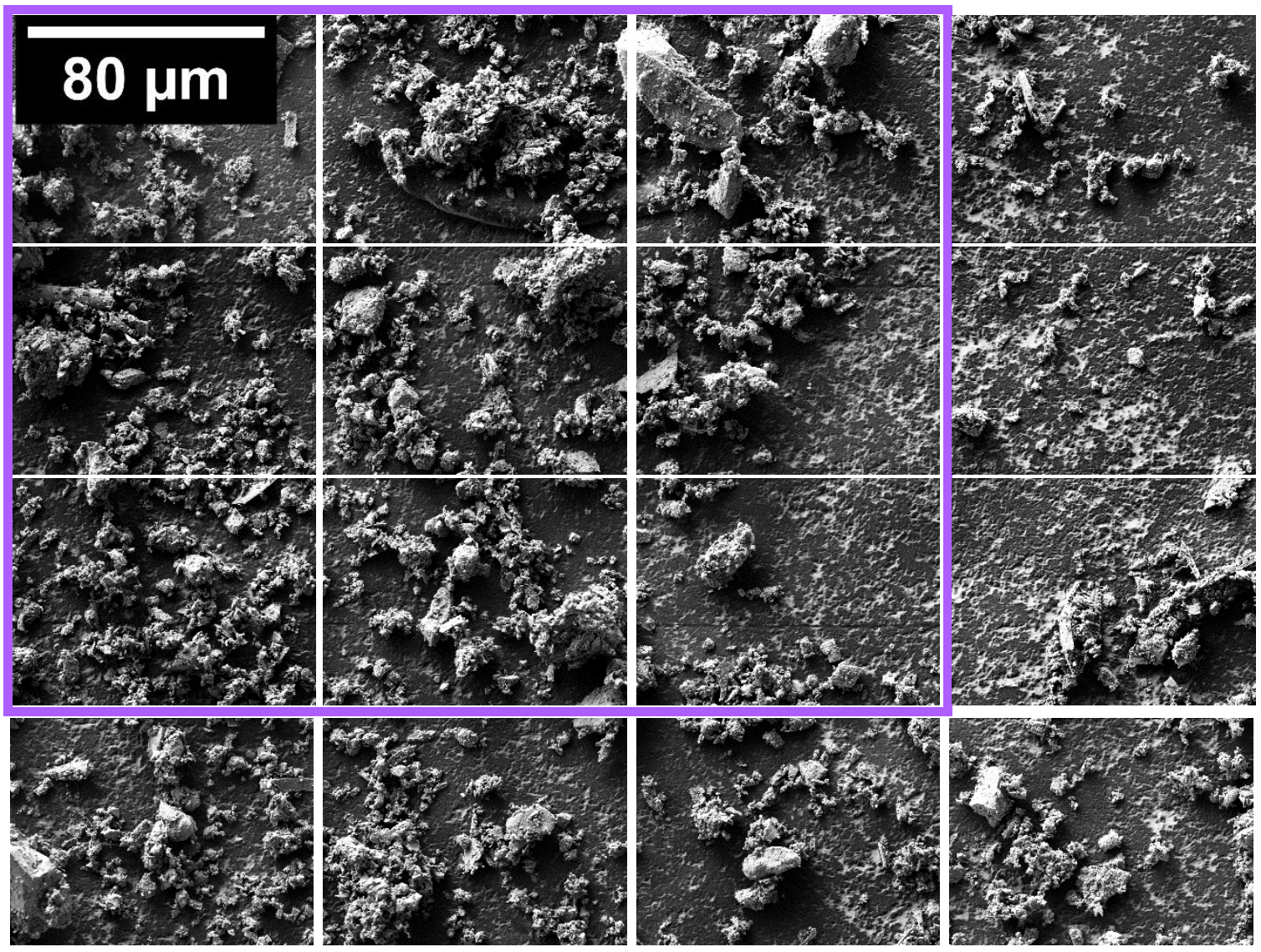

Figure 5-33. 4x4 image mosaic of original SEM images from specimen 01 from sample W1N10 from the Wickiup Junction, La Pine field site. The purple box illustrates the chosen $3 \times 3$ image mosaic chosen for analysis, Mosaic I. Images were captured at a magnification of 3000, and the scale bar of $80 \mu \mathrm{m}$ on the top right image applies for all images. 


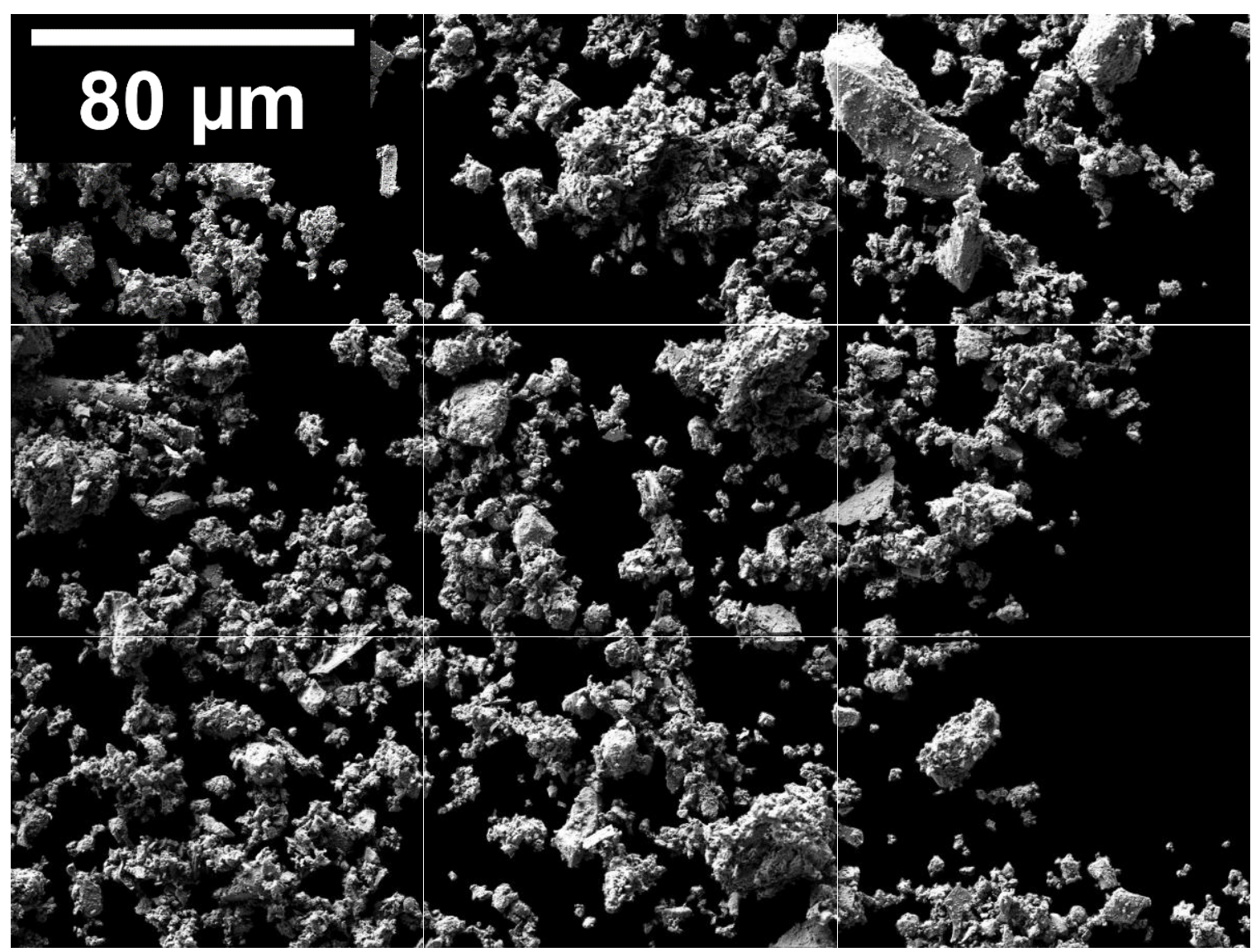

Figure 5-34. Mosaic I, $3 \times 3$ image mosaic of SEM images with all particles on a black background from specimen 01 from the Wickiup Junction, La Pine field site. Images were captured at a magnification of 3000 , and the scale bar of $80 \mu \mathrm{m}$ on the top right image applies for all images. 


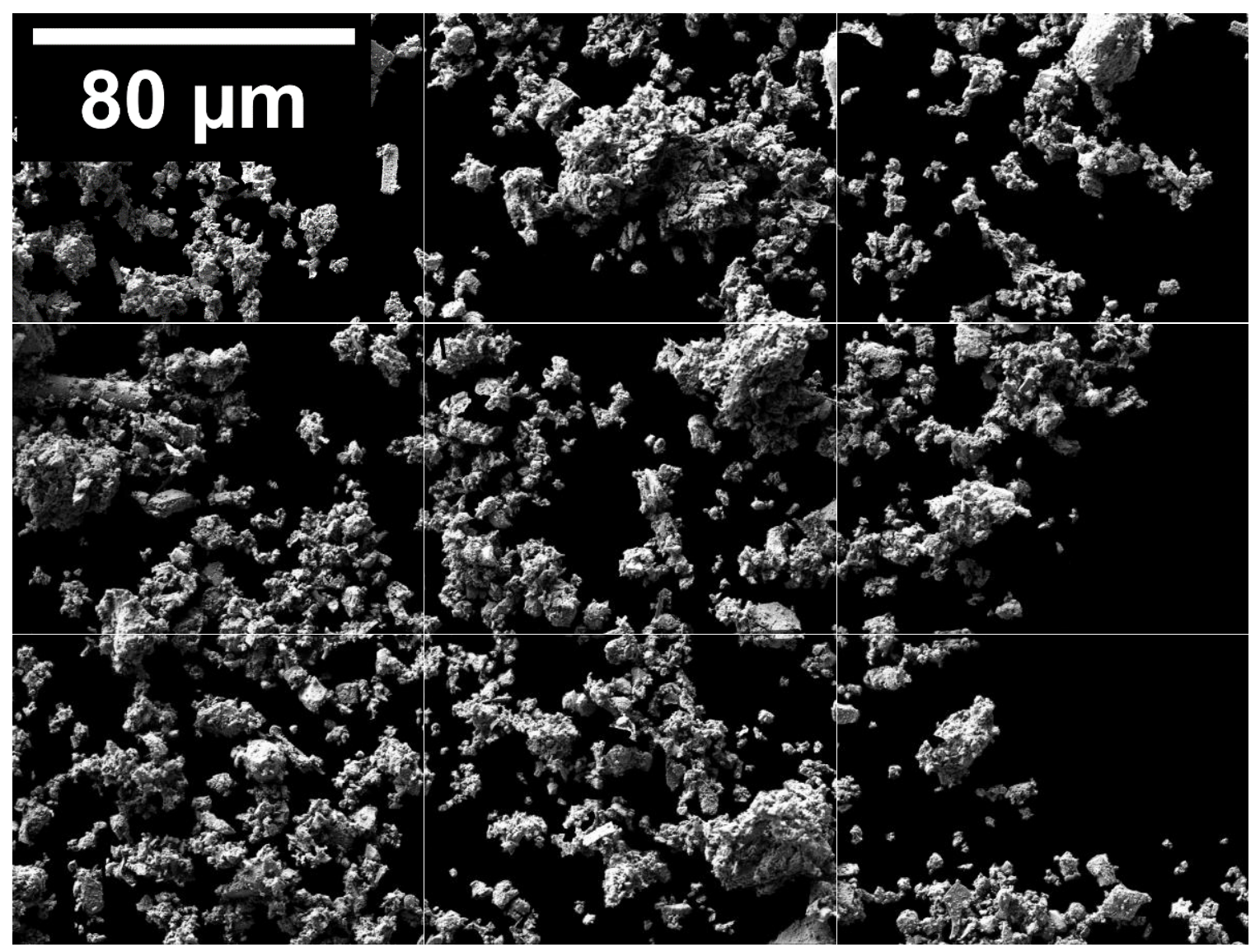

Figure 5-35. Mosaic I, $3 \times 3$ image mosaic of SEM images with only diatom particles on a black background from specimen 01 from the Wickiup Junction, La Pine field site. Images were captured at a magnification of 3000 , and the scale bar of $80 \mu \mathrm{m}$ on the top right image applies for all images. 


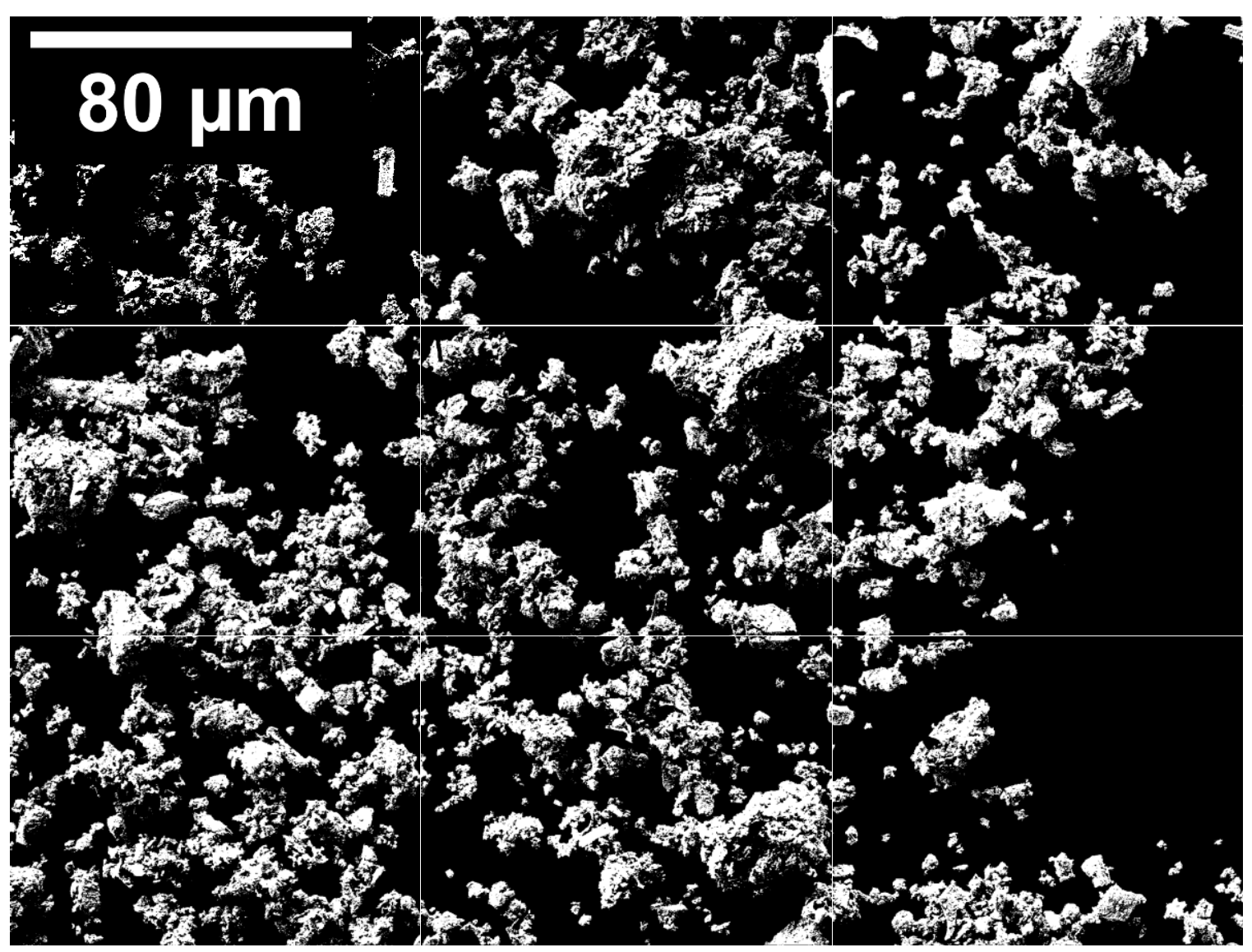

Figure 5-36. Mosaic I, $3 \times 3$ image mosaic of processed SEM images with only diatom particles on a black background from specimen 01 from the Wickiup Junction, La Pine field site. Images were captured at a magnification of 3000, and the scale bar of $80 \mu \mathrm{m}$ on the top right image applies for all images. 

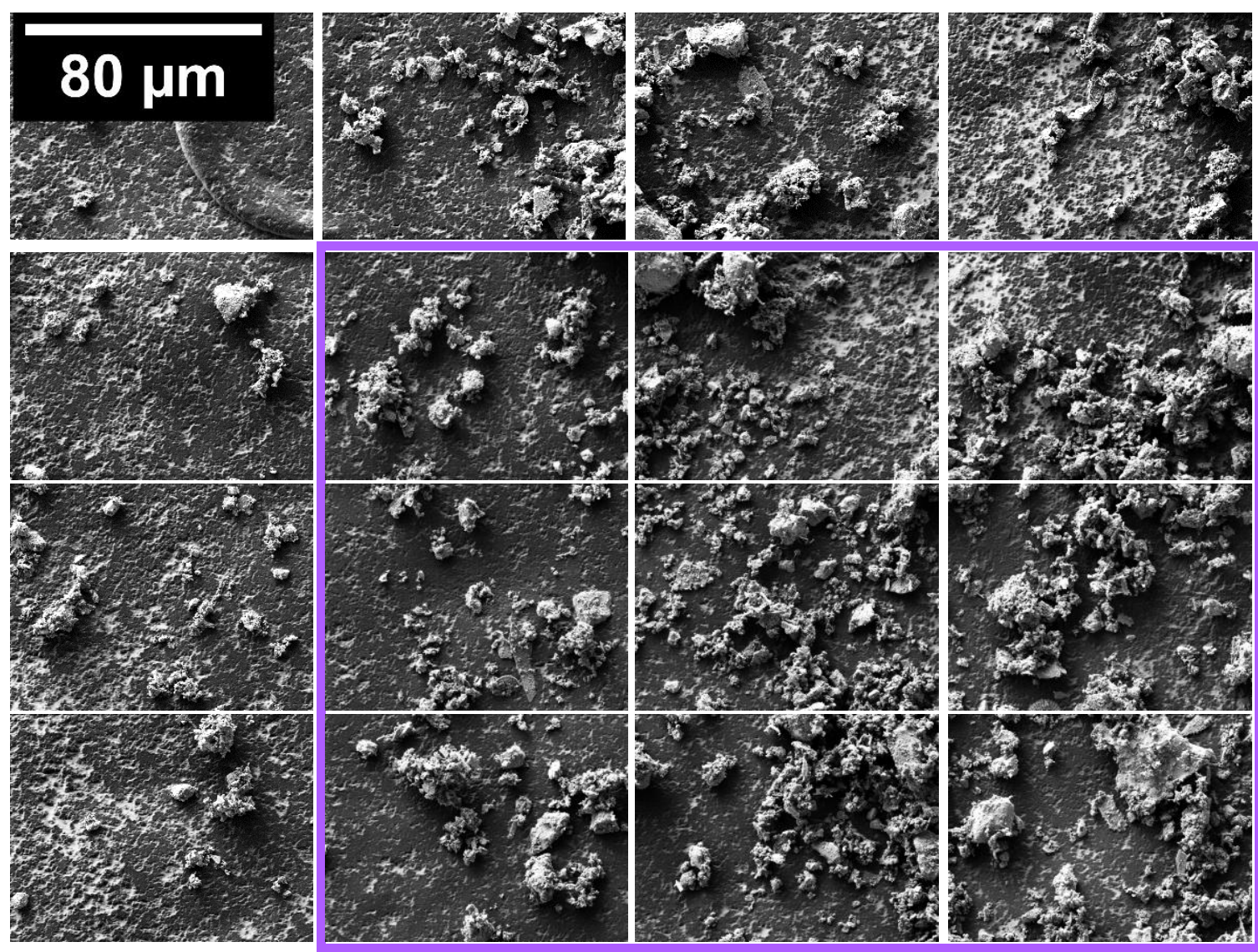

Figure 5-37. 4x4 image mosaic of original SEM images from specimen 01 from sample W1N10 from the Wickiup Junction, La Pine field site. The purple box illustrates the chosen $3 \times 3$ image mosaic chosen for analysis, Mosaic II. Images were captured at a magnification of 3000, and the scale bar of $80 \mu \mathrm{m}$ on the top right image applies for all images. 


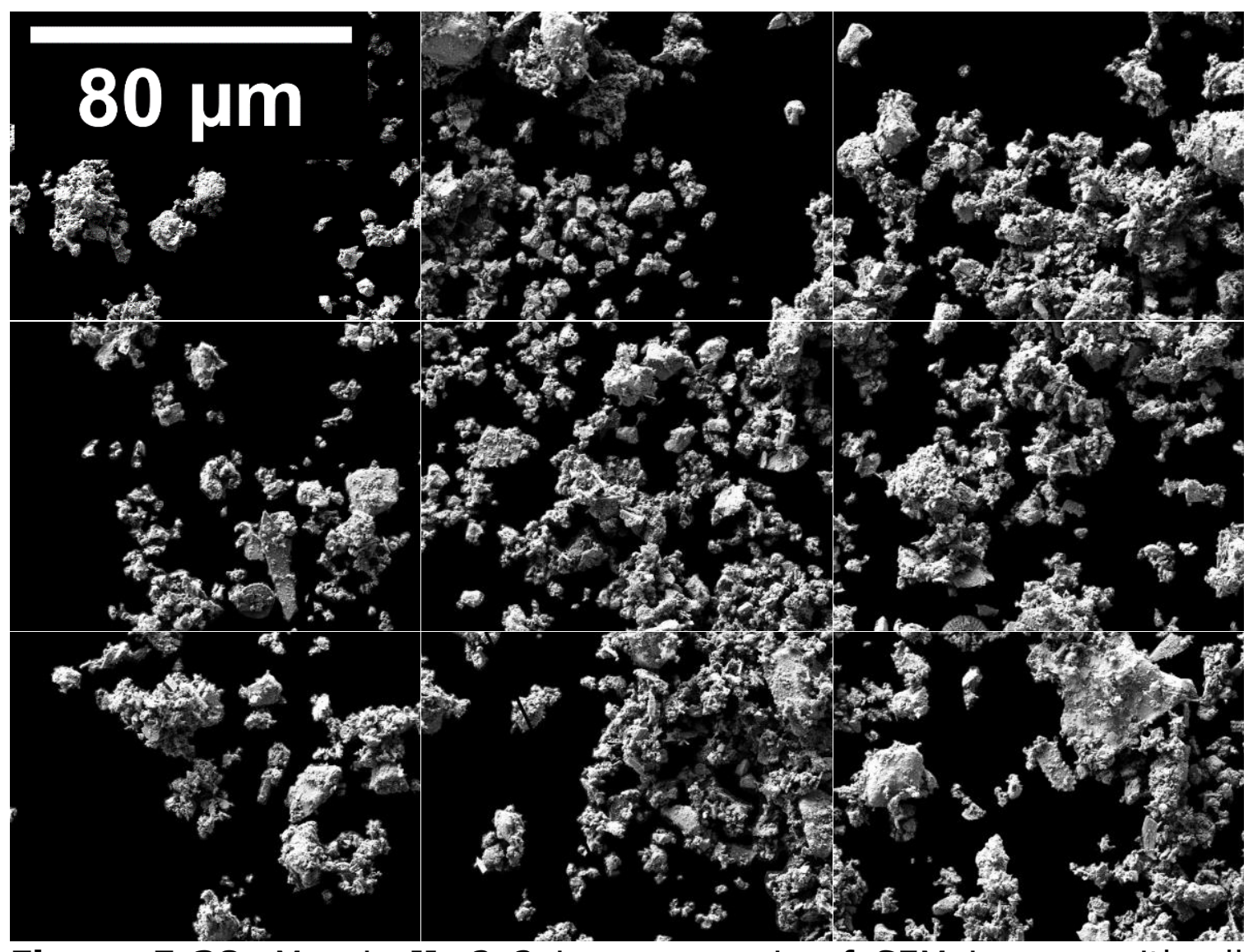

Figure 5-38. Mosaic II, $3 \times 3$ image mosaic of SEM images with all particles on a black background from specimen 01 from the Wickiup Junction, La Pine field site. Images were captured at a magnification of 3000 , and the scale bar of $80 \mu \mathrm{m}$ on the top right image applies for all images. 


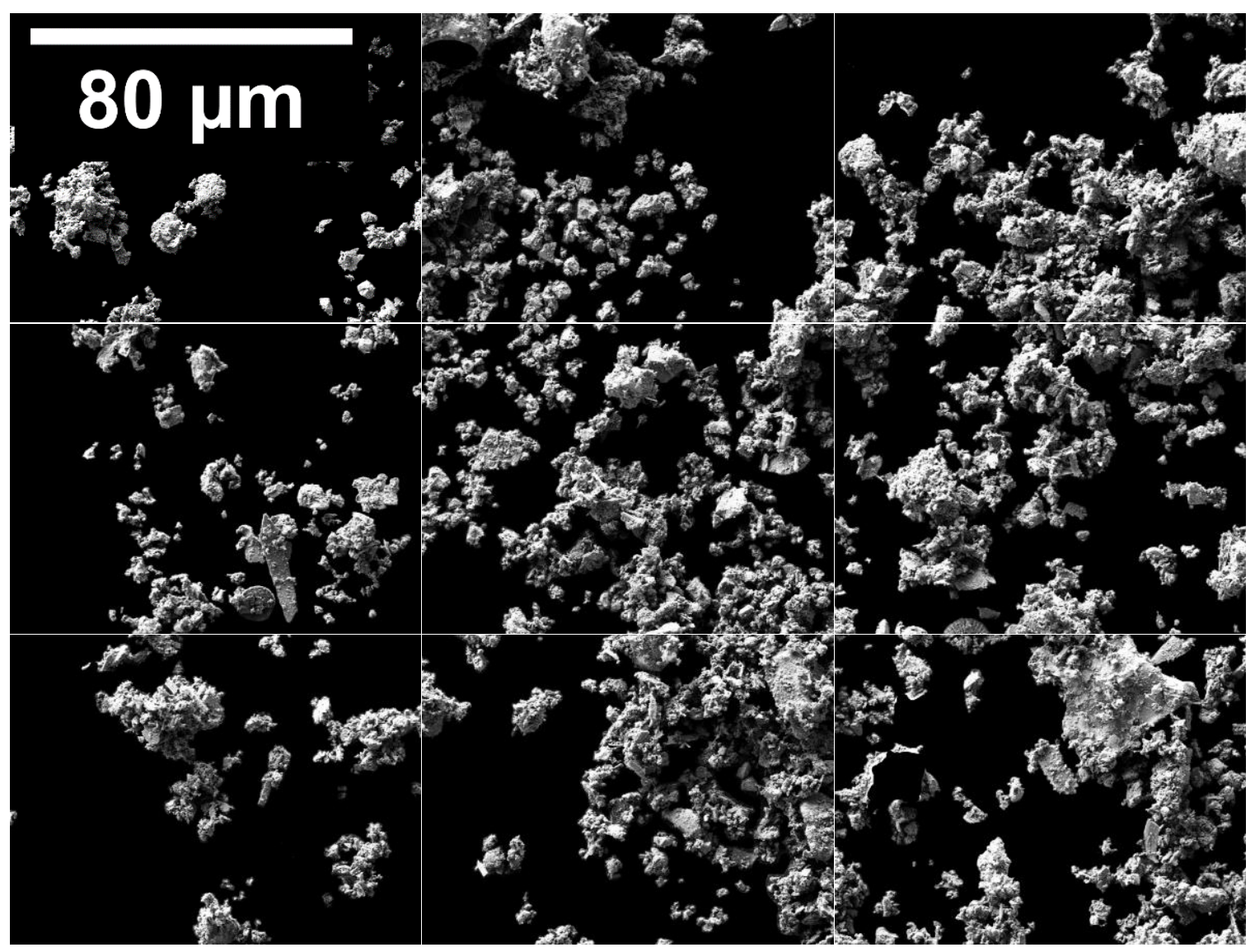

Figure 5-39. Mosaic II, $3 \times 3$ image mosaic of SEM images with only diatom particles on a black background from specimen 01 from the Wickiup Junction, La Pine field site. Images were captured at a magnification of 3000 , and the scale bar of $80 \mu \mathrm{m}$ on the top right image applies for all images. 


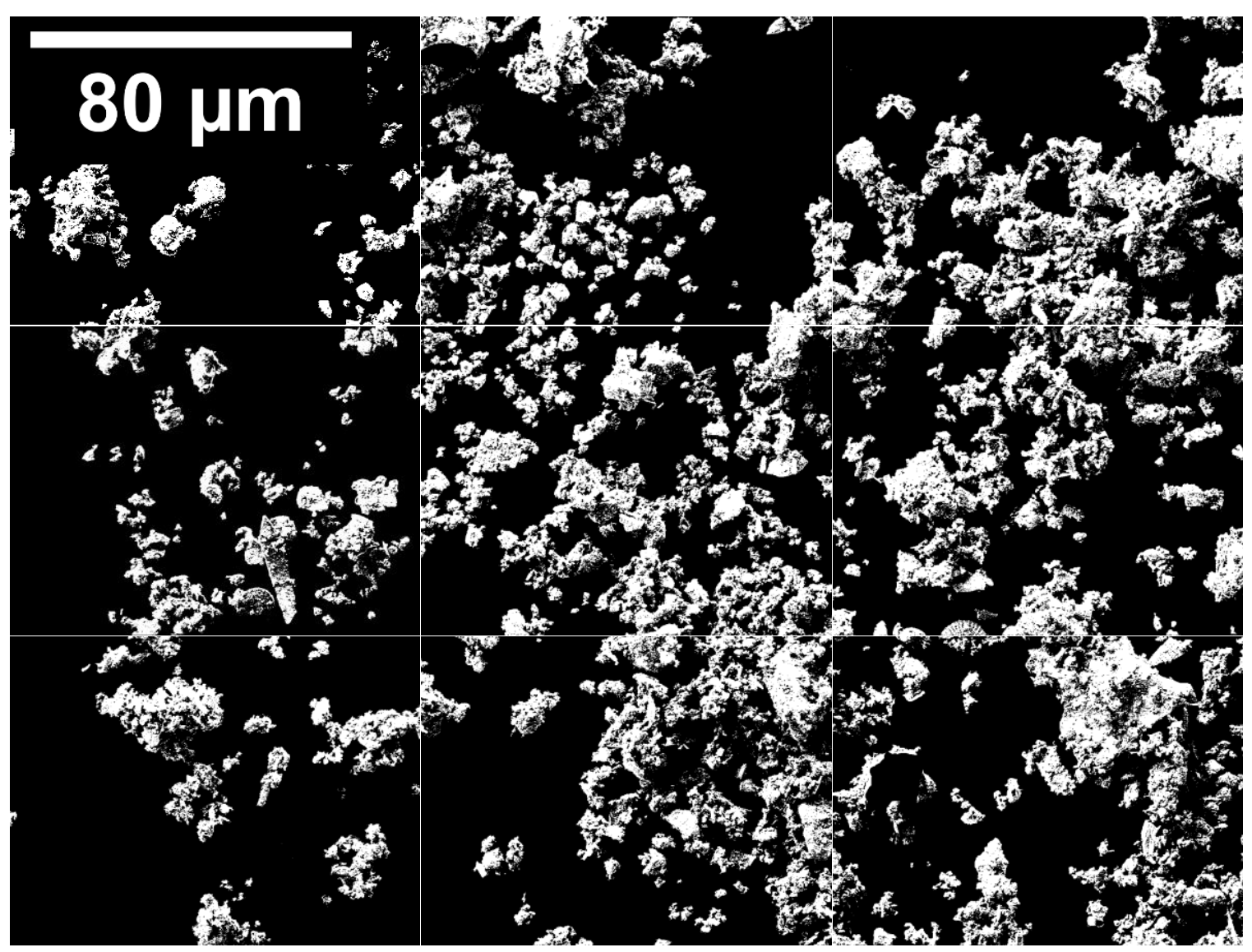

Figure 5-40. Mosaic II, $3 \times 3$ image mosaic of processed SEM images with only diatom particles on a black background from specimen 01 from the Wickiup Junction, La Pine field site. Images were captured at a magnification of 3000, and the scale bar of $80 \mu \mathrm{m}$ on the top right image applies for all images. 


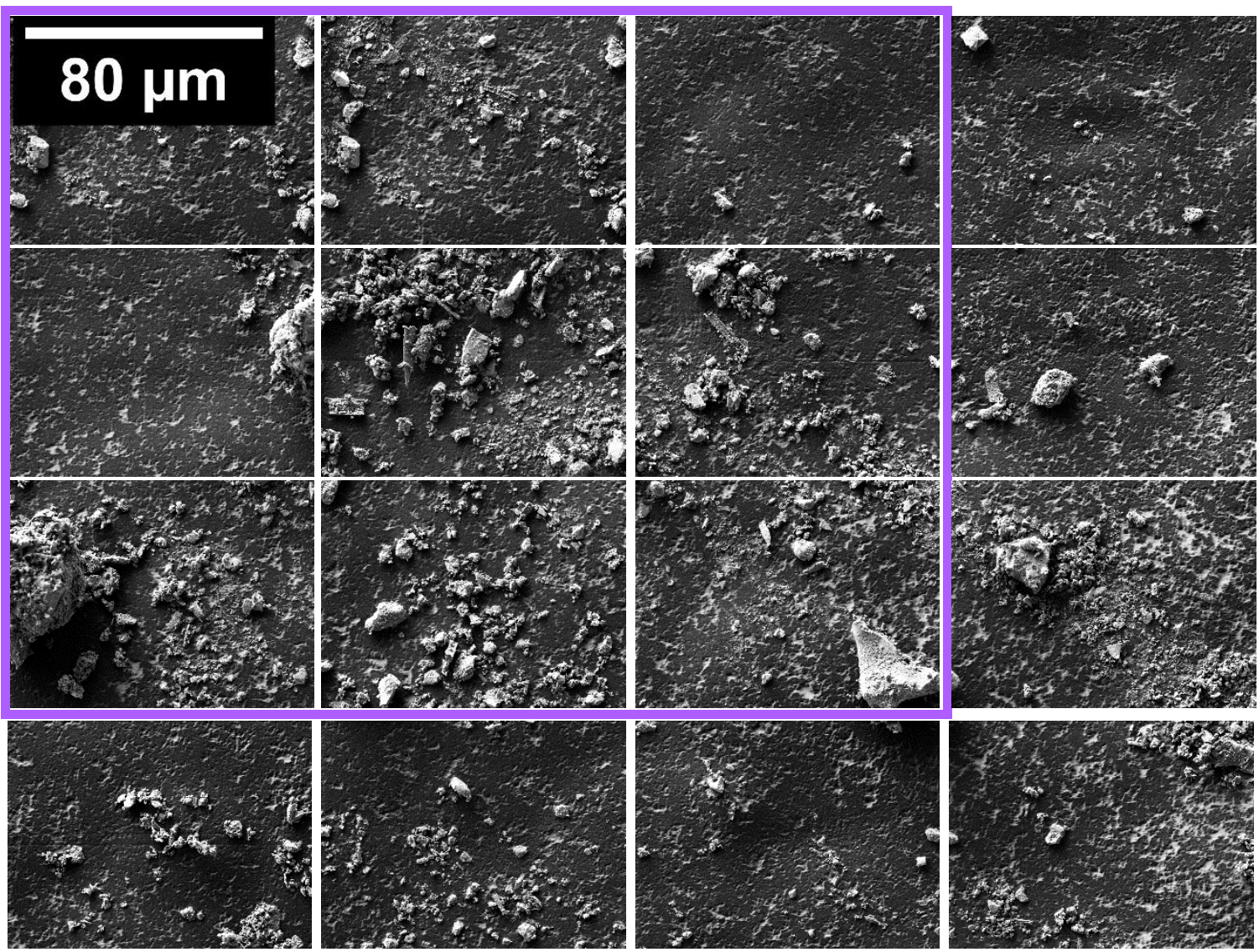

Figure 5-41. 4x4 image mosaic of original SEM images from specimen 01 from sample W1N10 from the Wickiup Junction, La Pine field site. The purple box illustrates the chosen $3 \times 3$ image mosaic chosen for analysis, Mosaic III. Images were captured at a magnification of 3000, and the scale bar of $80 \mu \mathrm{m}$ on the top right image applies for all images. 


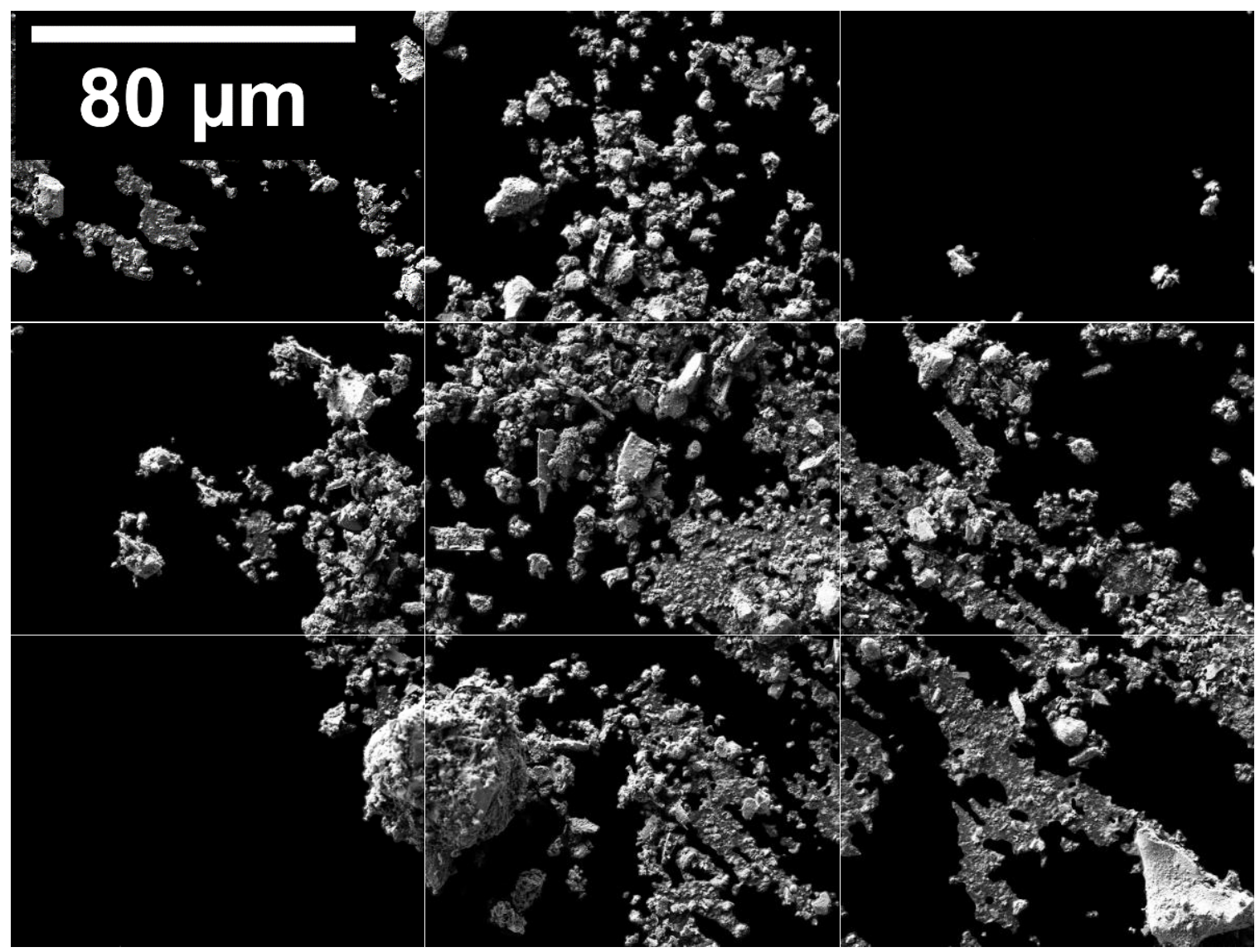

Figure 5-42. Mosaic III, $3 \times 3$ image mosaic of SEM images with all particles on a black background from specimen 01 from the Wickiup Junction, La Pine field site. Images were captured at a magnification of 3000 , and the scale bar of $80 \mu \mathrm{m}$ on the top right image applies for all images. 


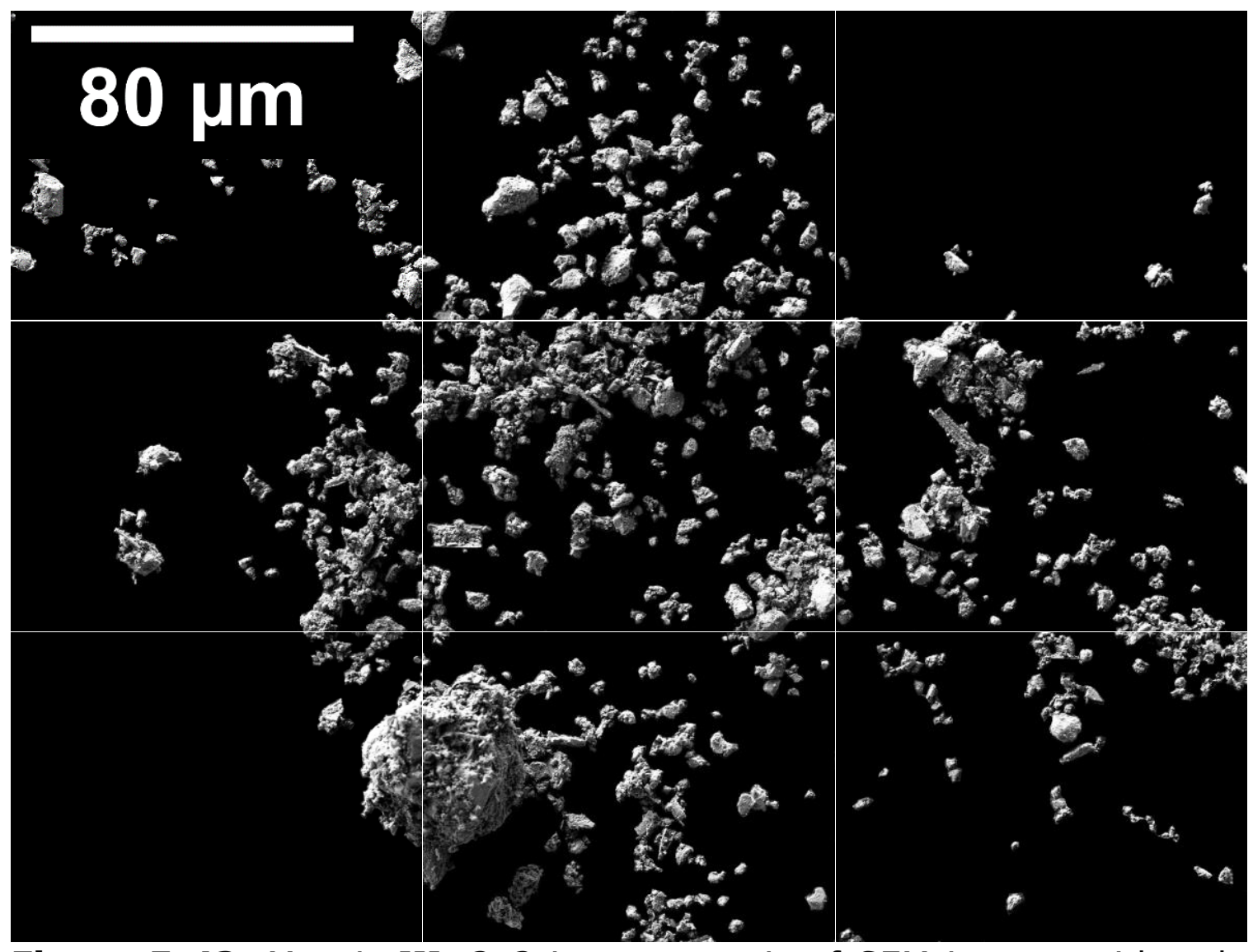

Figure 5-43. Mosaic III, $3 \times 3$ image mosaic of SEM images with only diatom particles on a black background from specimen 01 from the Wickiup Junction, La Pine field site. Images were captured at a magnification of 3000 , and the scale bar of $80 \mu \mathrm{m}$ on the top right image applies for all images. 


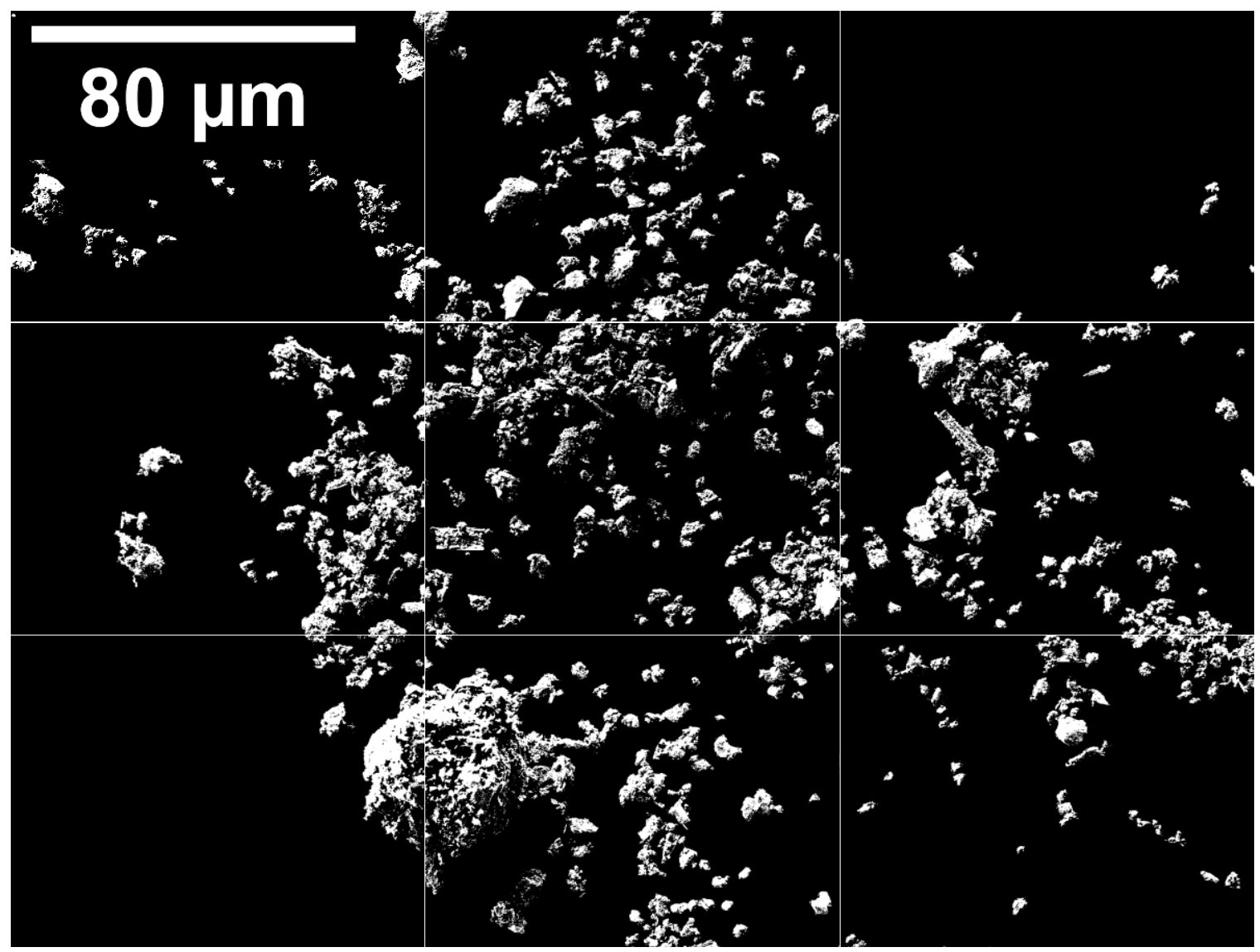

Figure 5-44. Mosaic III, $3 \times 3$ image mosaic of processed SEM images with only diatom particles on a black background from specimen 01 from the Wickiup Junction, La Pine field site. Images were captured at a magnification of 3000, and the scale bar of $80 \mu \mathrm{m}$ on the top right image applies for all images. 


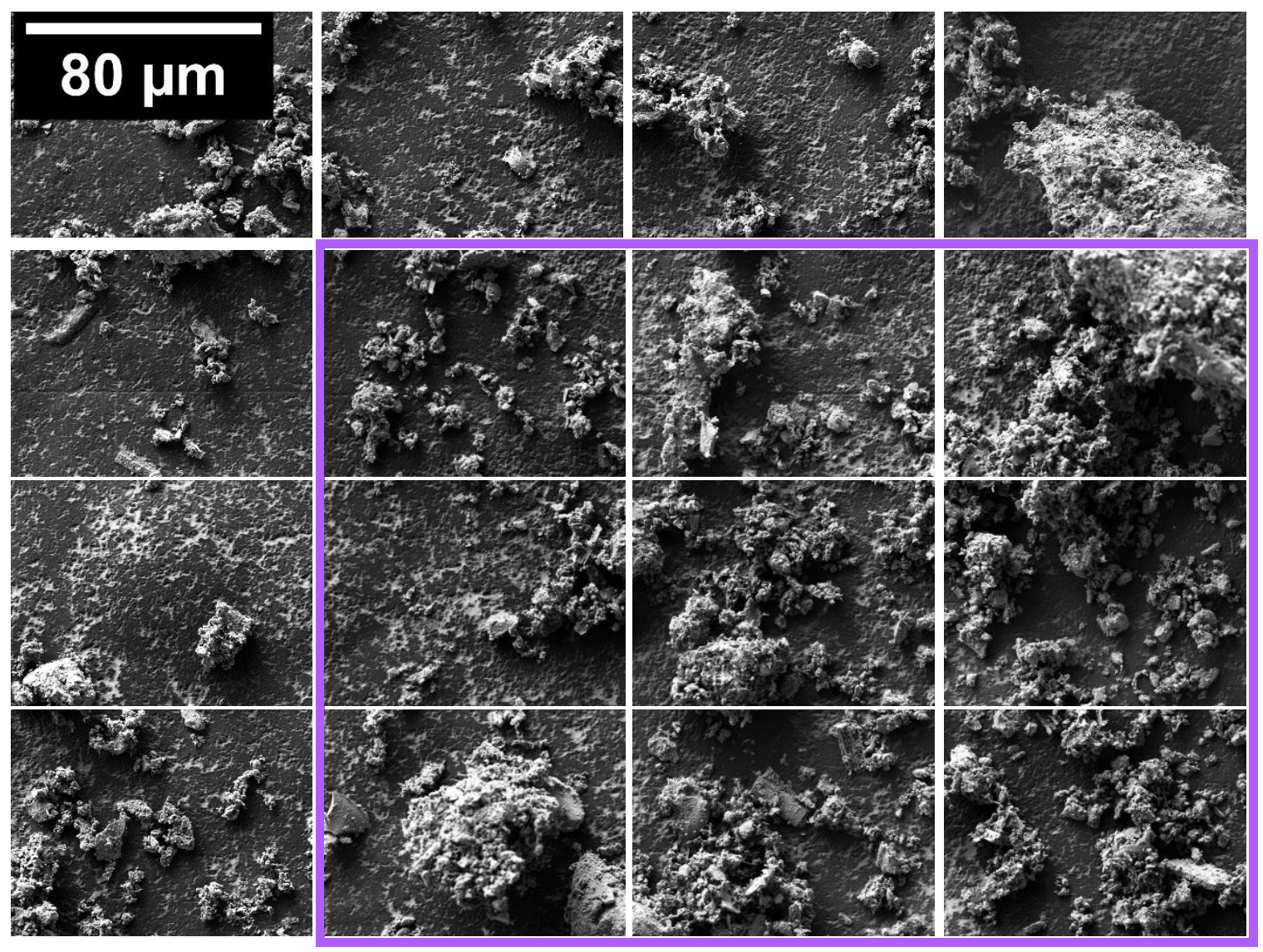

Figure 5-45. 4x4 image mosaic of original SEM images from specimen 01 from sample W1N10 from the Wickiup Junction, La Pine field site. The purple box illustrates the chosen $3 \times 3$ image mosaic chosen for analysis, Mosaic IV. Images were captured at a magnification of 3000, and the scale bar of $80 \mu \mathrm{m}$ on the top right image applies for all images. 


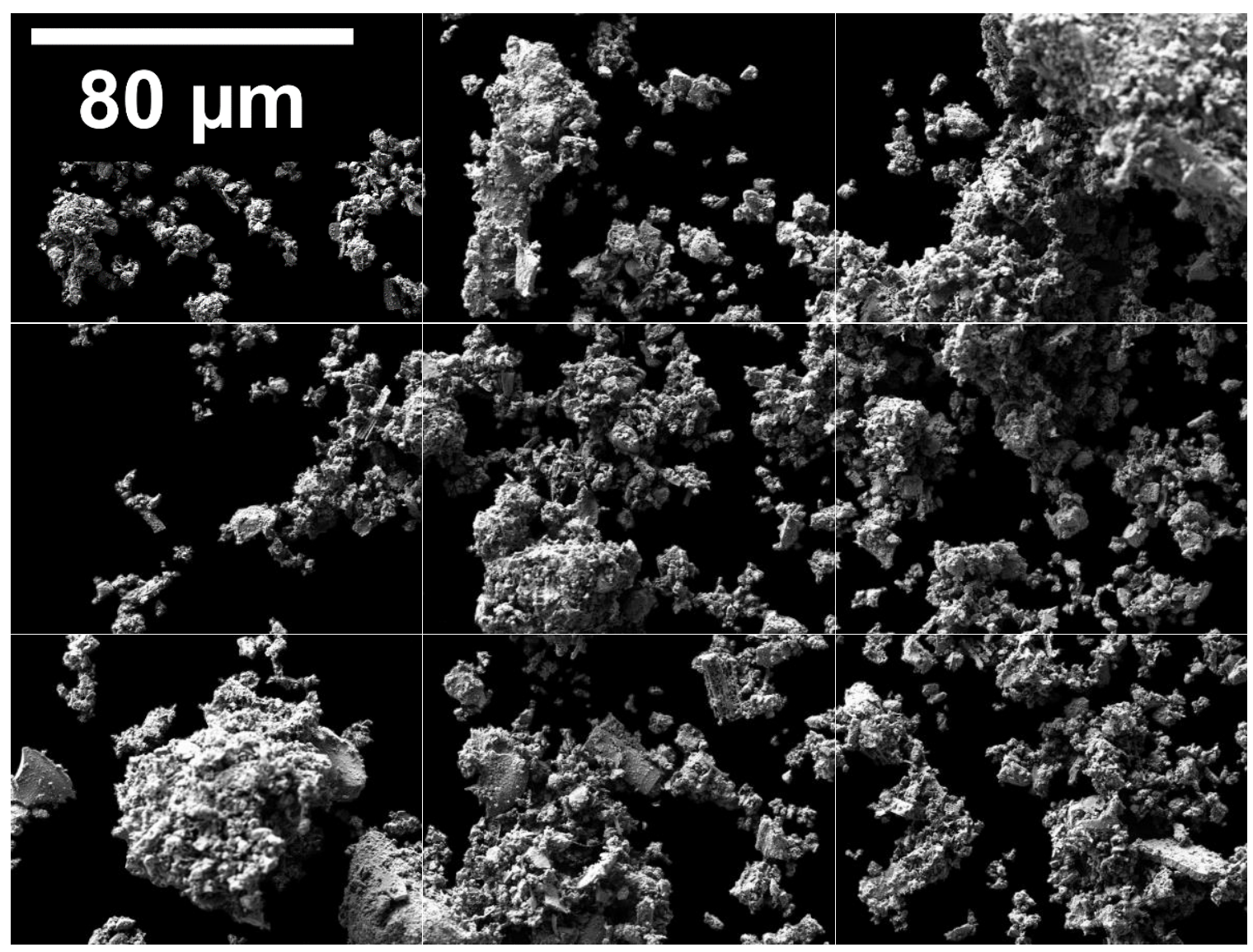

Figure 5-46. Mosaic IV, $3 \times 3$ image mosaic of SEM images with all particles on a black background from specimen 01 from the Wickiup Junction, La Pine field site. Images were captured at a magnification of 3000 , and the scale bar of $80 \mu \mathrm{m}$ on the top right image applies for all images. 


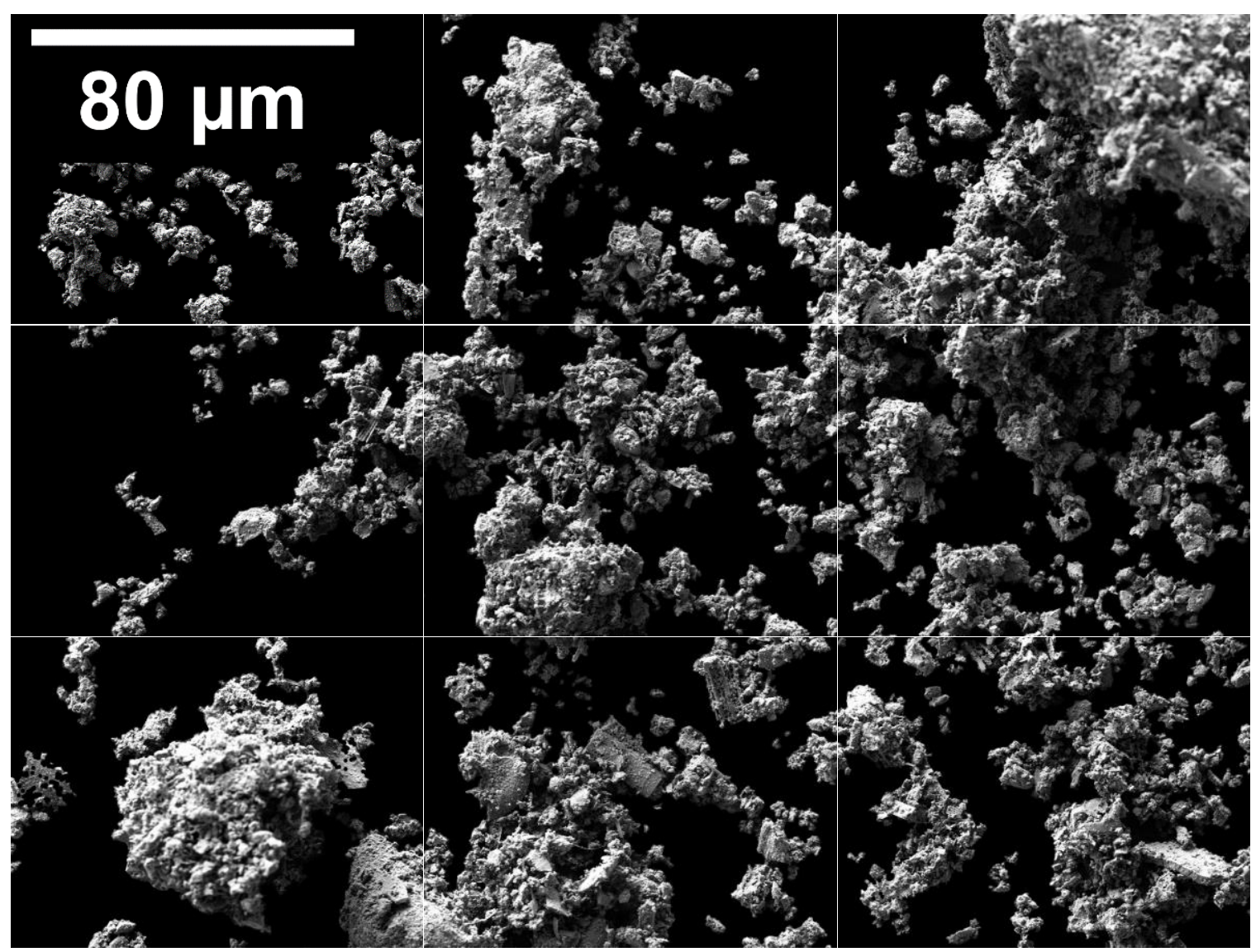

Figure 5-47. Mosaic IV, $3 \times 3$ image mosaic of SEM images with only diatom particles on a black background from specimen 01 from the Wickiup Junction, La Pine field site. Images were captured at a magnification of 3000 , and the scale bar of $80 \mu \mathrm{m}$ on the top right image applies for all images. 


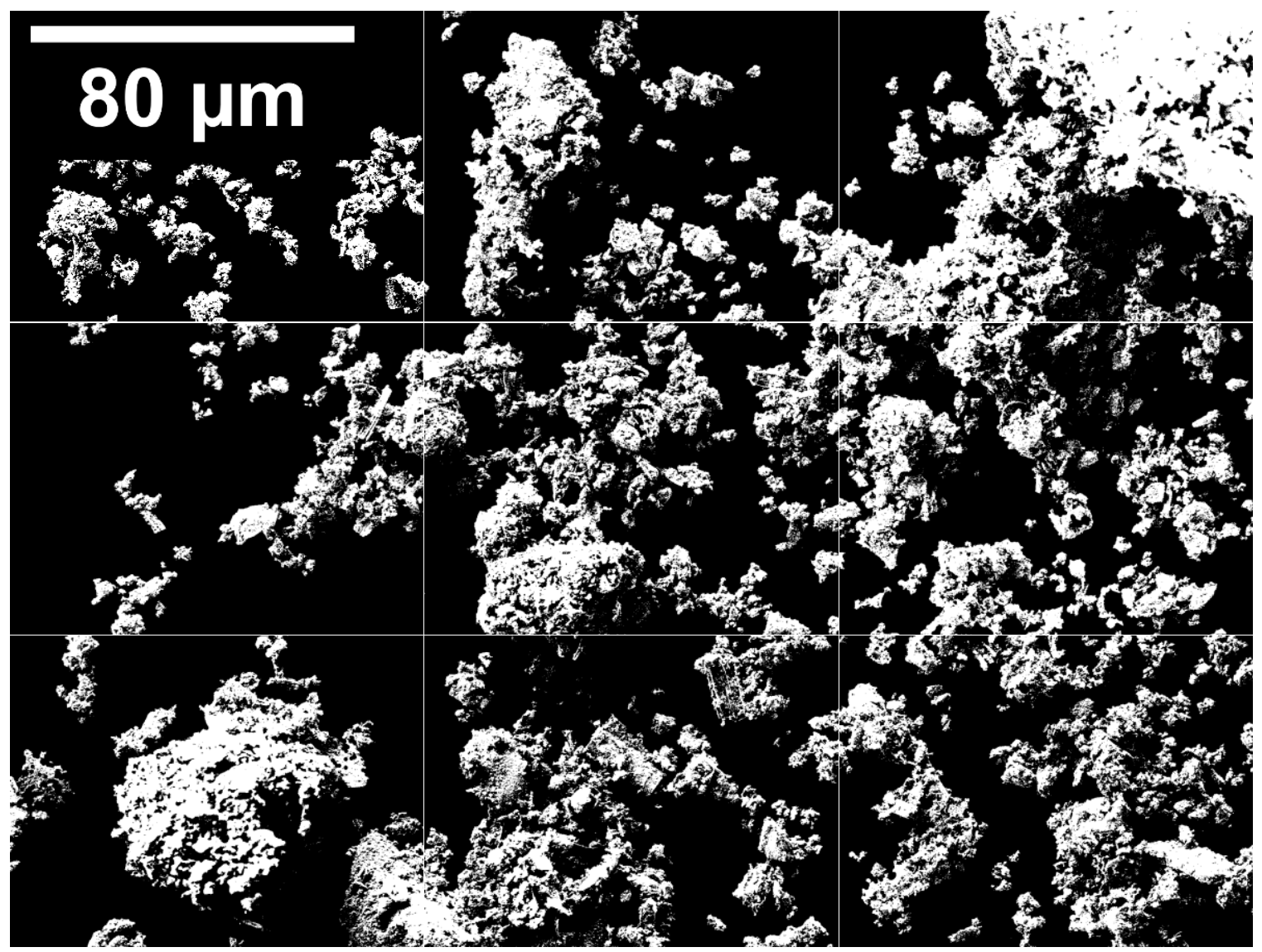

Figure 5-48. Mosaic IV, $3 \times 3$ image mosaic of processed SEM images with only diatom particles on a black background from specimen 01 from the Wickiup Junction, La Pine field site. Images were captured at a magnification of 3000, and the scale bar of $80 \mu \mathrm{m}$ on the top right image applies for all images. 


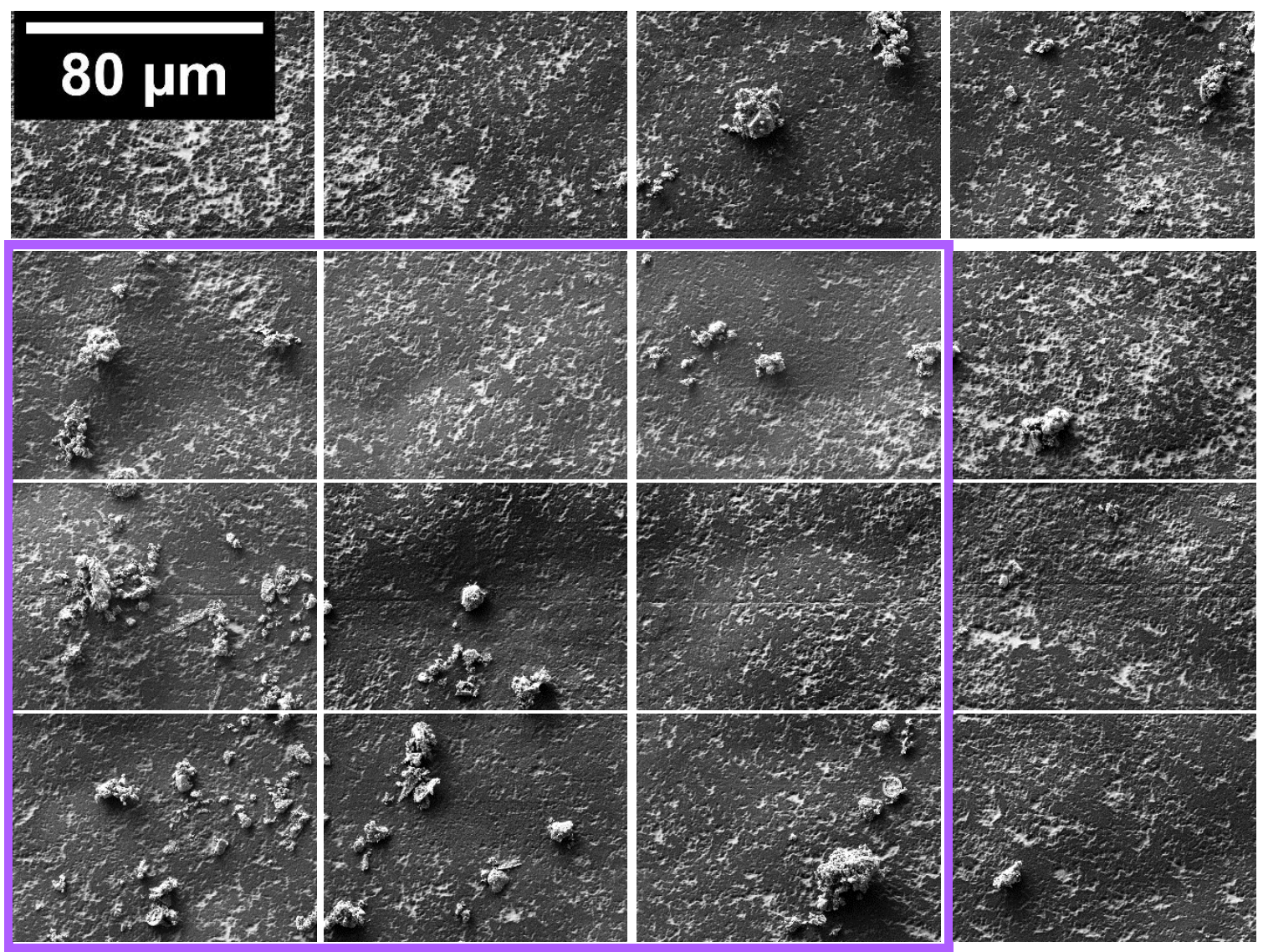

Figure 5-49. $4 \times 4$ image mosaic of original SEM images from specimen 02 from sample W1N10 from the Wickiup Junction, La Pine field site. The purple box illustrates the chosen $3 \times 3$ image mosaic chosen for analysis, Mosaic I. Images were captured at a magnification of 3000, and the scale bar of $80 \mu \mathrm{m}$ on the top right image applies for all images. 


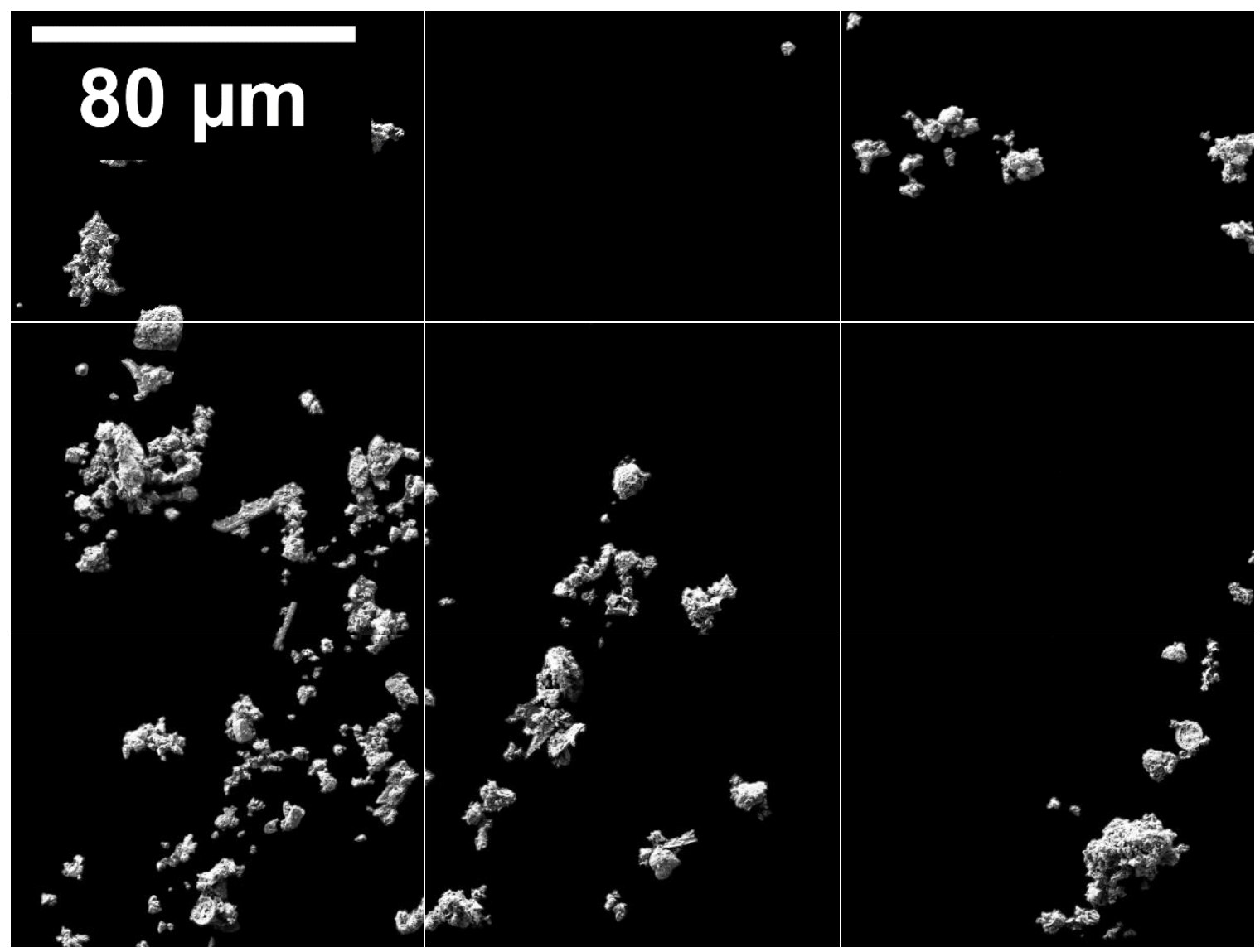

Figure 5-50. Mosaic I, $3 \times 3$ image mosaic of SEM images with all particles on a black background from specimen 02 from the Wickiup Junction, La Pine field site. Images were captured at a magnification of 3000 , and the scale bar of $80 \mu \mathrm{m}$ on the top right image applies for all images. 


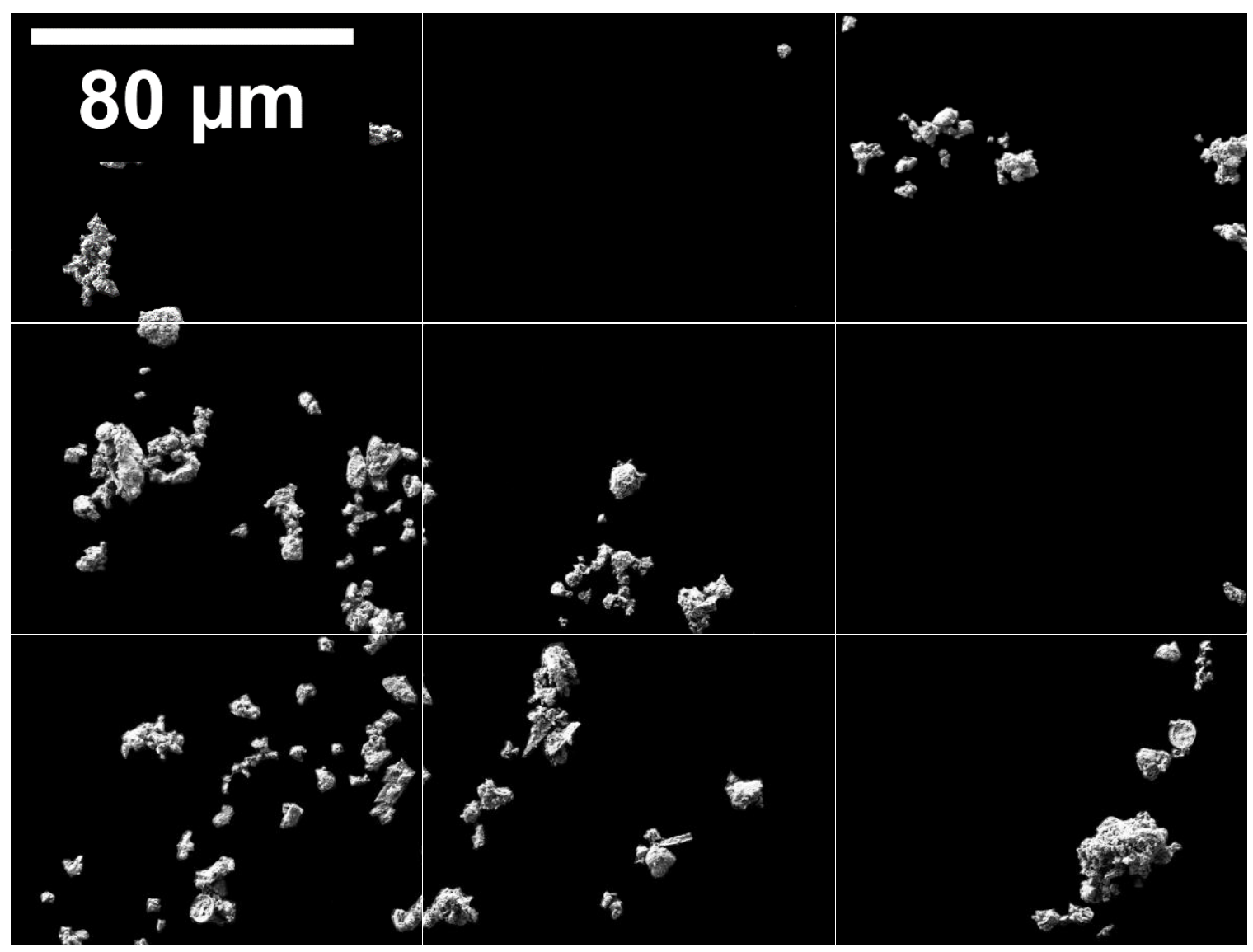

Figure 5-51. Mosaic I, $3 \times 3$ image mosaic of SEM images with only diatom particles on a black background from specimen 02 from the Wickiup Junction, La Pine field site. Images were captured at a magnification of 3000 , and the scale bar of $80 \mu \mathrm{m}$ on the top right image applies for all images. 


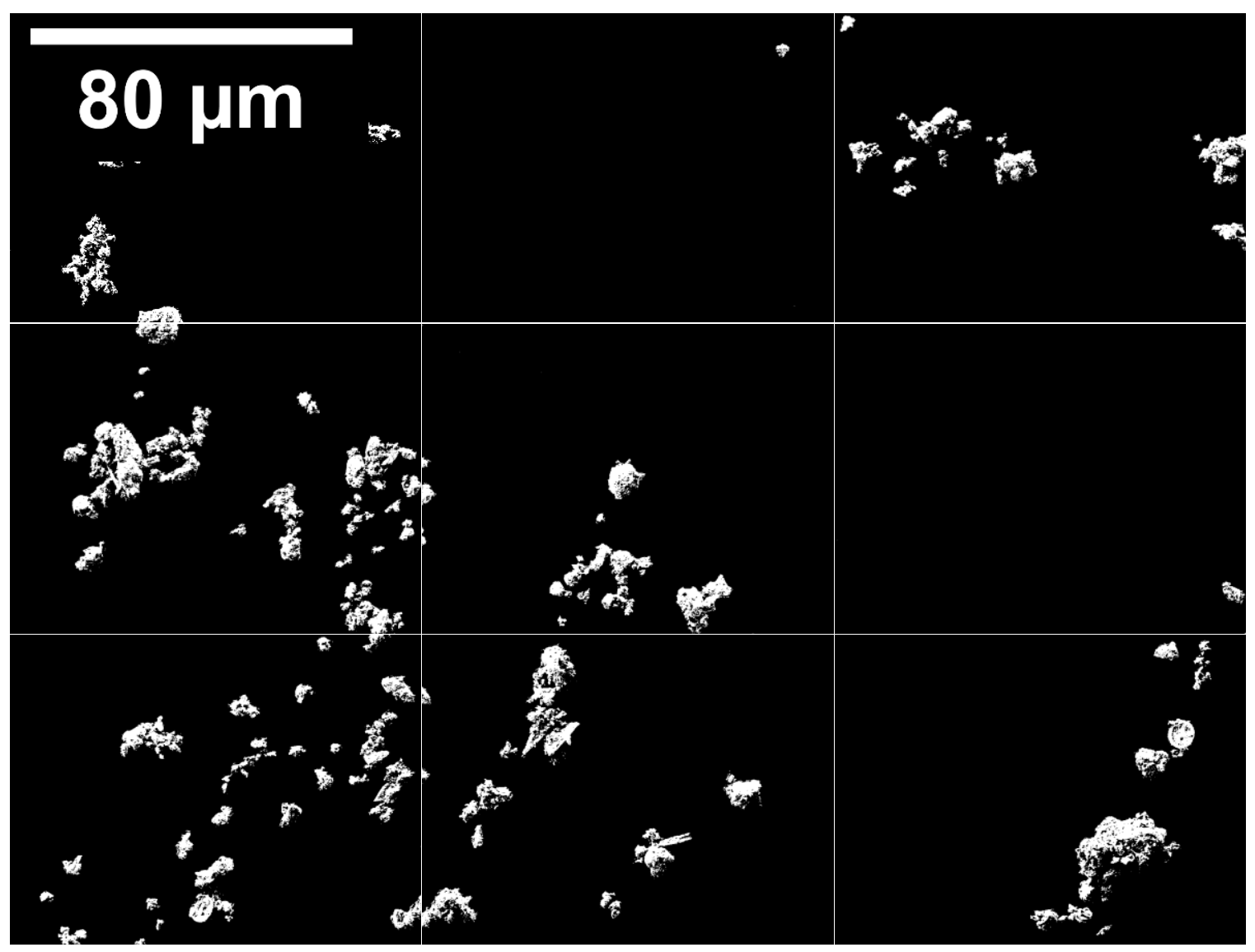

Figure 5-52. Mosaic I, $3 \times 3$ image mosaic of processed SEM images with only diatom particles on a black background from specimen 02 from the Wickiup Junction, La Pine field site. Images were captured at a magnification of 3000, and the scale bar of $80 \mu \mathrm{m}$ on the top right image applies for all images. 


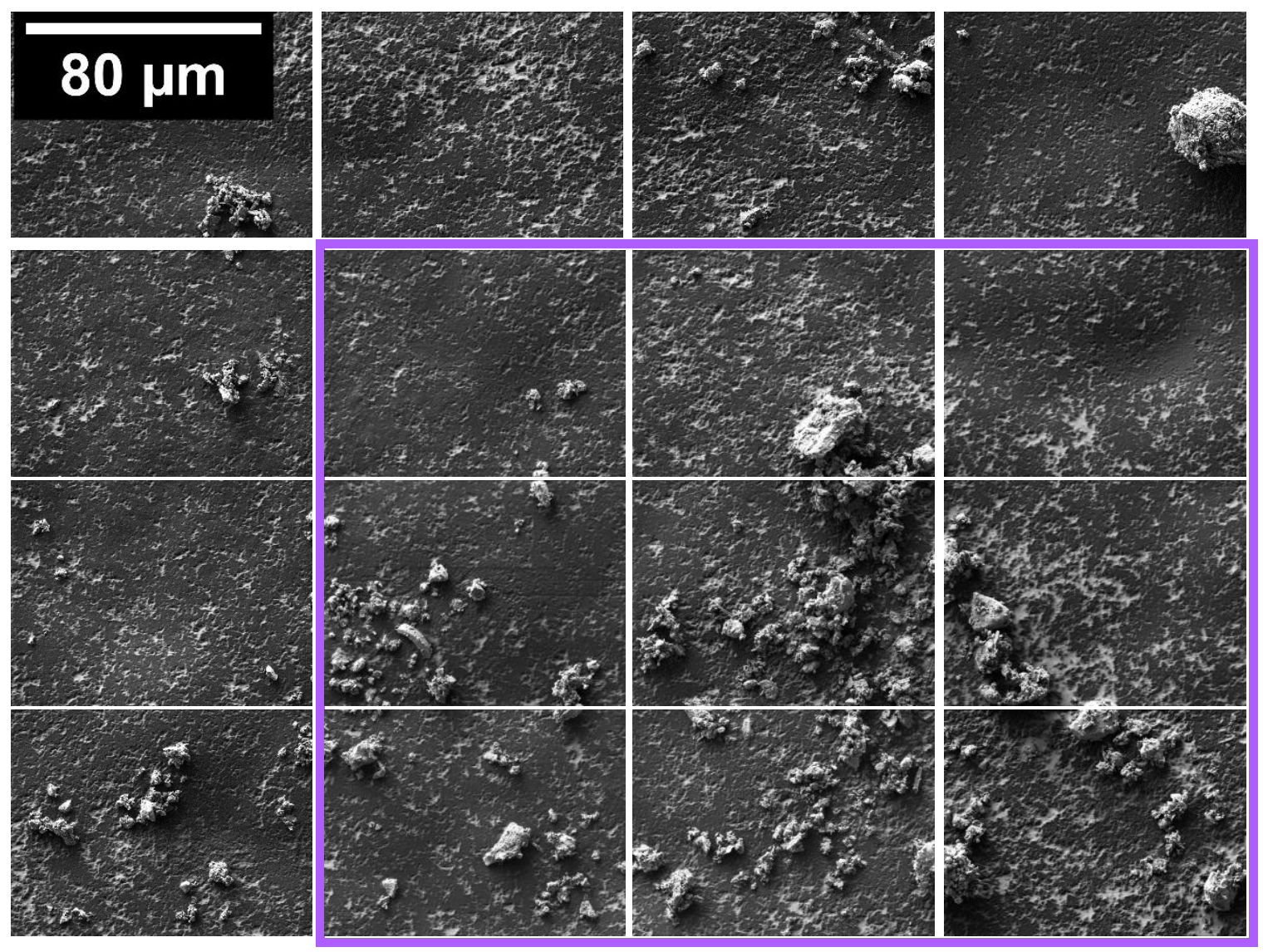

Figure 5-53. $4 \times 4$ image mosaic of original SEM images from specimen 02 from sample W1N10 from the Wickiup Junction, La Pine field site. The purple box illustrates the chosen $3 \times 3$ image mosaic chosen for analysis, Mosaic II. Images were captured at a magnification of 3000, and the scale bar of $80 \mu \mathrm{m}$ on the top right image applies for all images. 


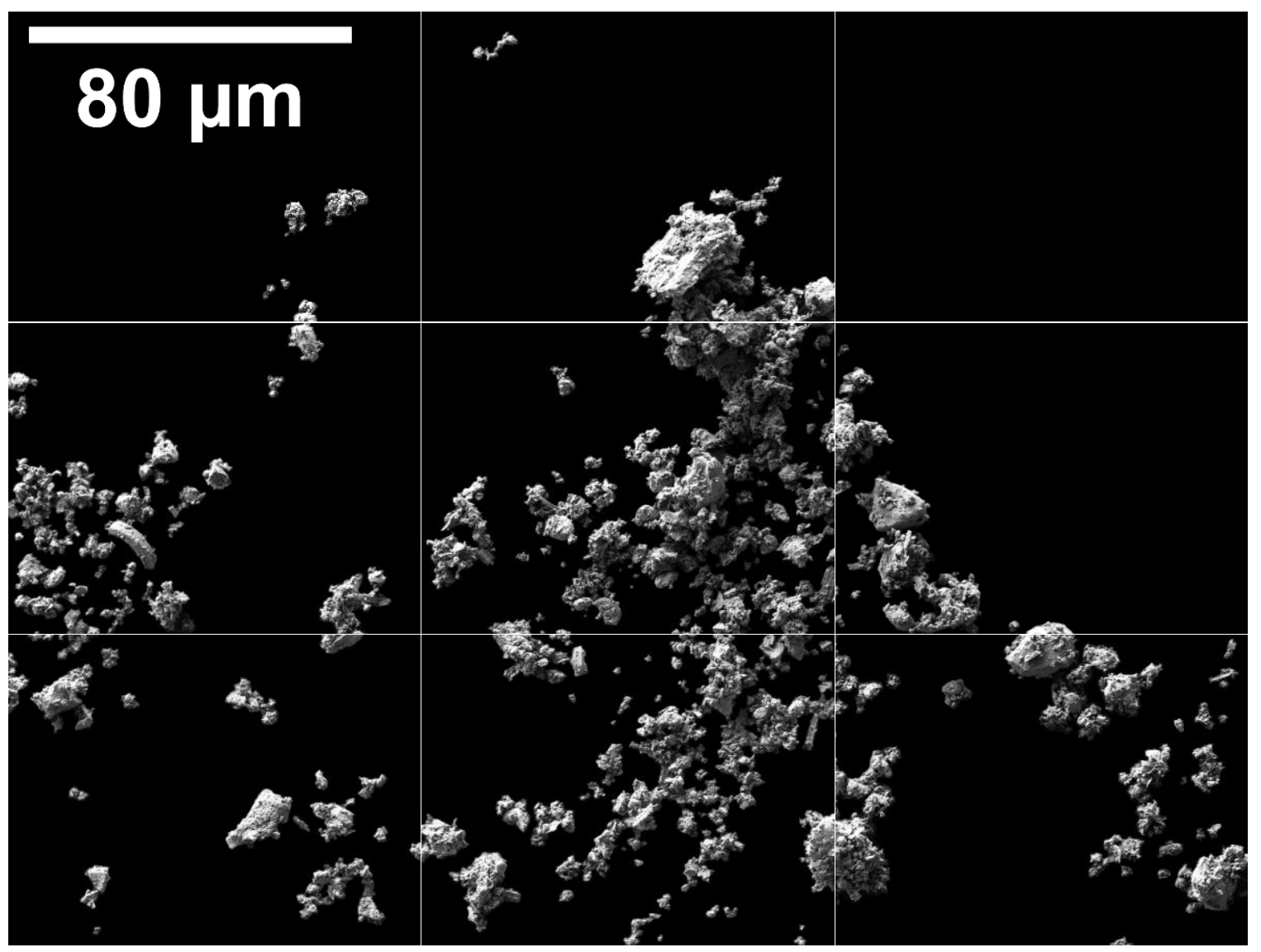

Figure 5-54. Mosaic II, $3 \times 3$ image mosaic of SEM images with all particles on a black background from specimen 02 from the Wickiup Junction, La Pine field site. Images were captured at a magnification of 3000 , and the scale bar of $80 \mu \mathrm{m}$ on the top right image applies for all images. 


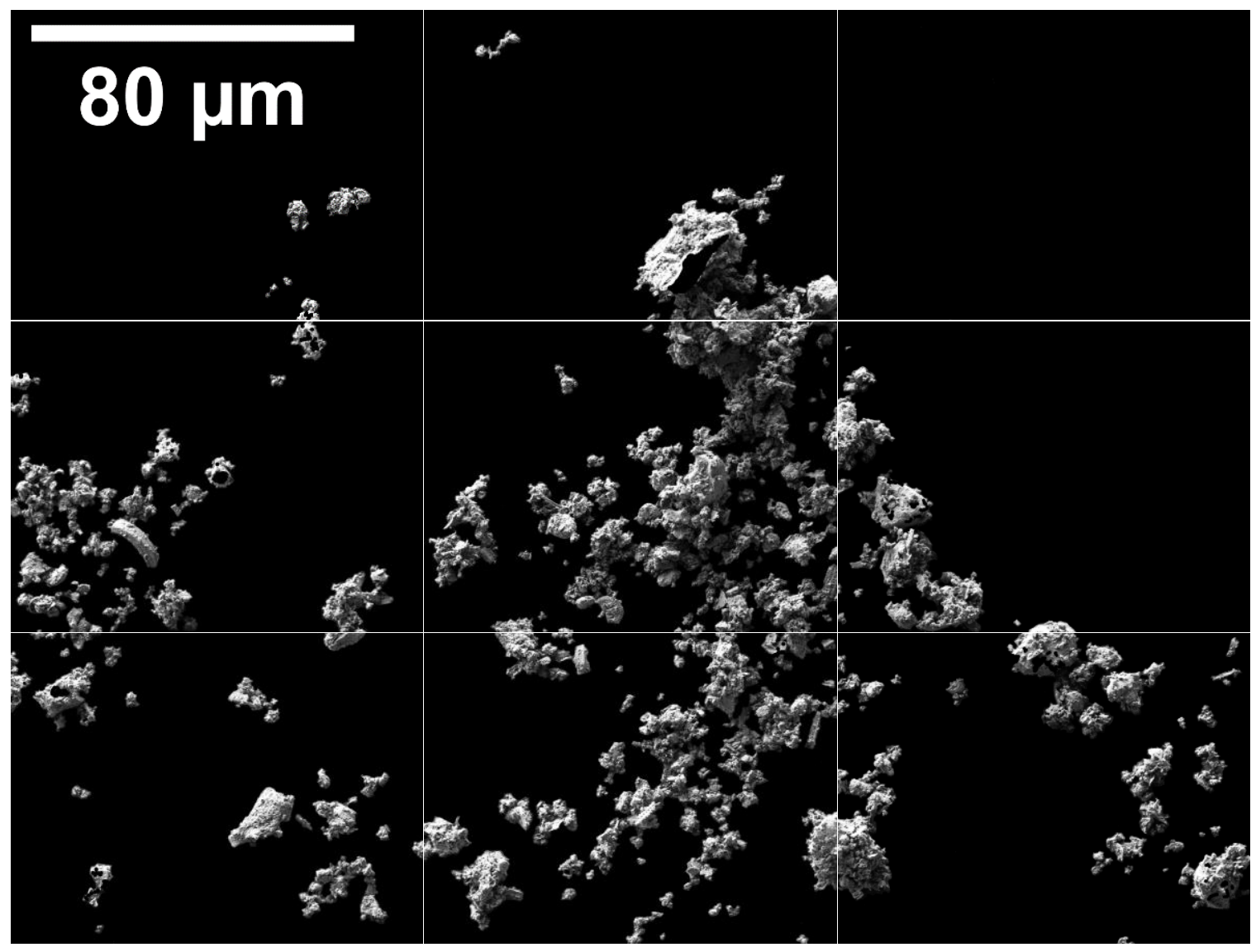

Figure 5-55. Mosaic II, $3 \times 3$ image mosaic of SEM images with only diatom particles on a black background from specimen 02 from the Wickiup Junction, La Pine field site. Images were captured at a magnification of 3000 , and the scale bar of $80 \mu \mathrm{m}$ on the top right image applies for all images. 


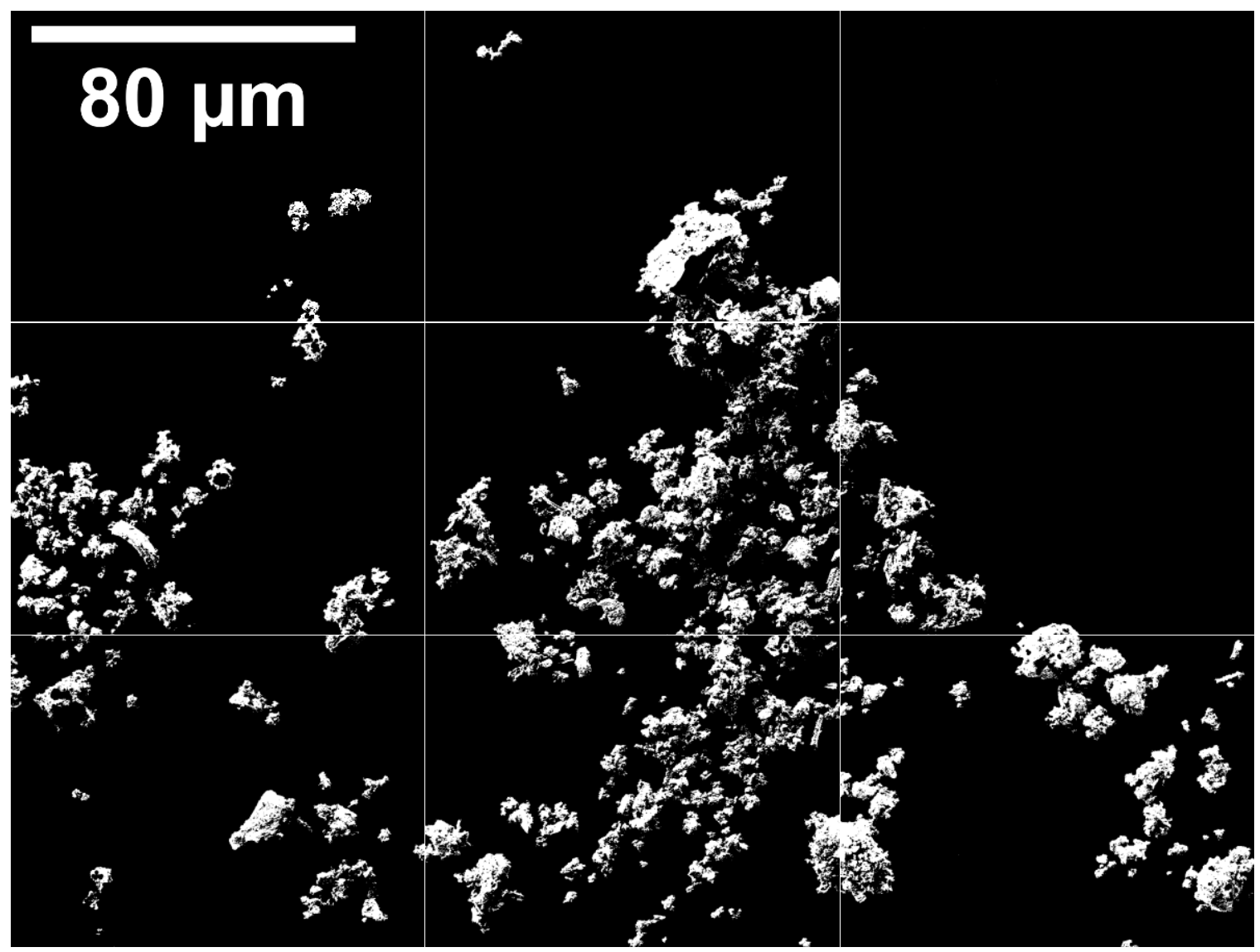

Figure 5-56. Mosaic II, $3 \times 3$ image mosaic of processed SEM images with only diatom particles on a black background from specimen 02 from the Wickiup Junction, La Pine field site. Images were captured at a magnification of 3000, and the scale bar of $80 \mu \mathrm{m}$ on the top right image applies for all images. 

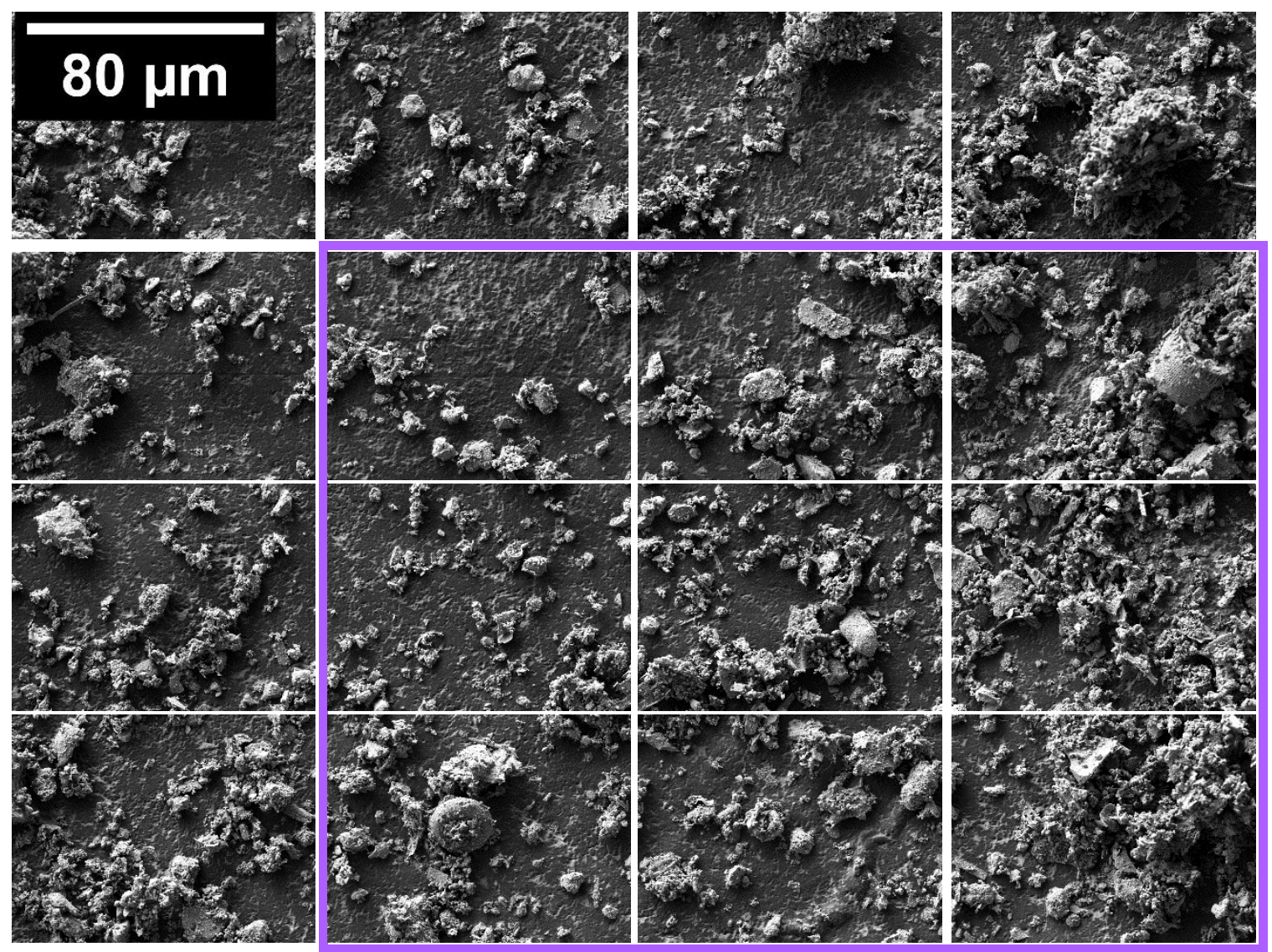

Figure 5-57. $4 \times 4$ image mosaic of original SEM images from specimen

02 from sample W1N10 from the Wickiup Junction, La Pine field site. The purple box illustrates the chosen $3 \times 3$ image mosaic chosen for analysis, Mosaic III. Images were captured at a magnification of 3000, and the scale bar of $80 \mu \mathrm{m}$ on the top right image applies for all images. 


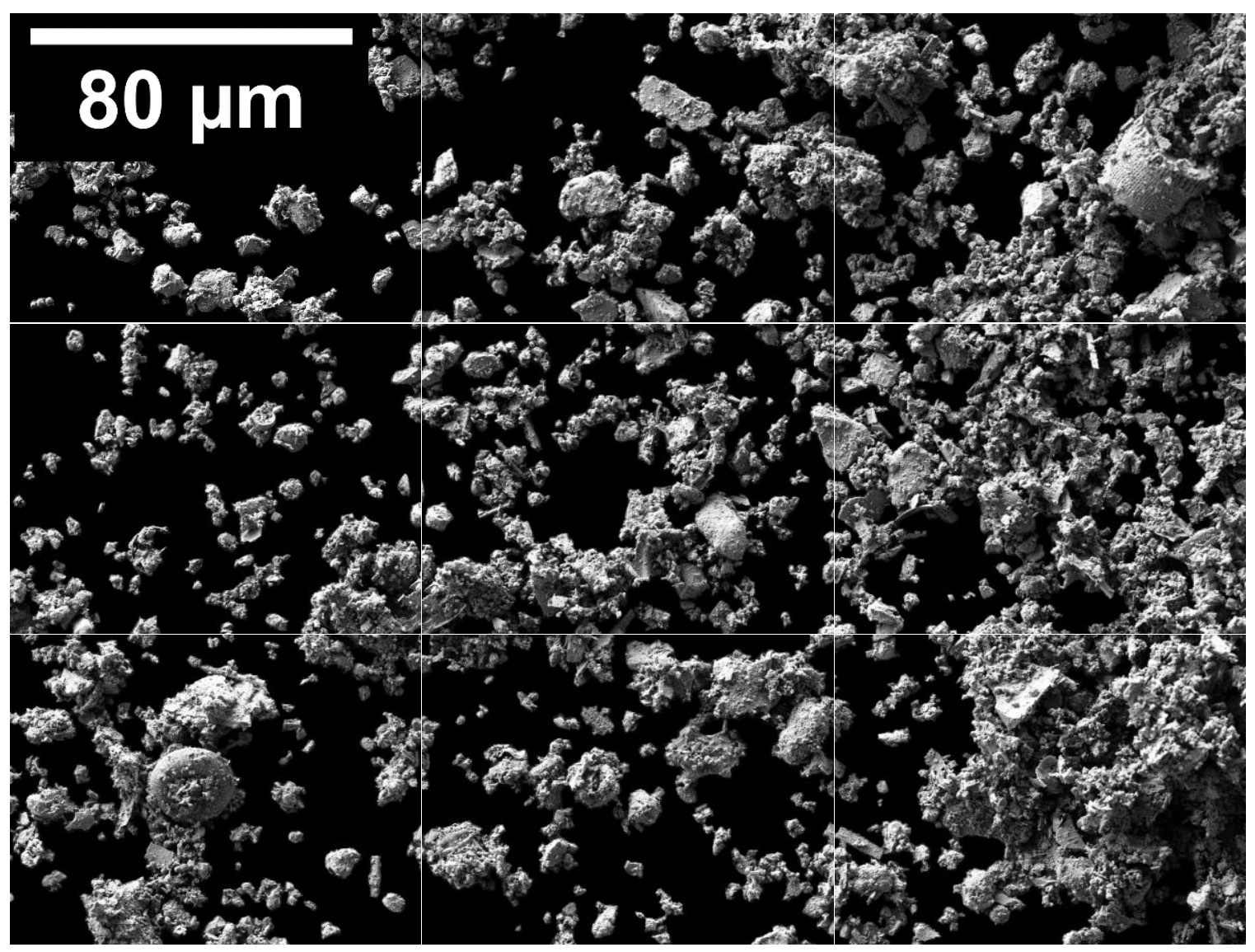

Figure 5-58. Mosaic III, $3 \times 3$ image mosaic of SEM images with all particles on a black background from specimen 02 from the Wickiup Junction, La Pine field site. Images were captured at a magnification of 3000 , and the scale bar of $80 \mu \mathrm{m}$ on the top right image applies for all images. 




Figure 5-59. Mosaic III, $3 \times 3$ image mosaic of SEM images with only diatom particles on a black background from specimen 02 from the Wickiup Junction, La Pine field site. Images were captured at a magnification of 3000 , and the scale bar of $80 \mu \mathrm{m}$ on the top right image applies for all images. 


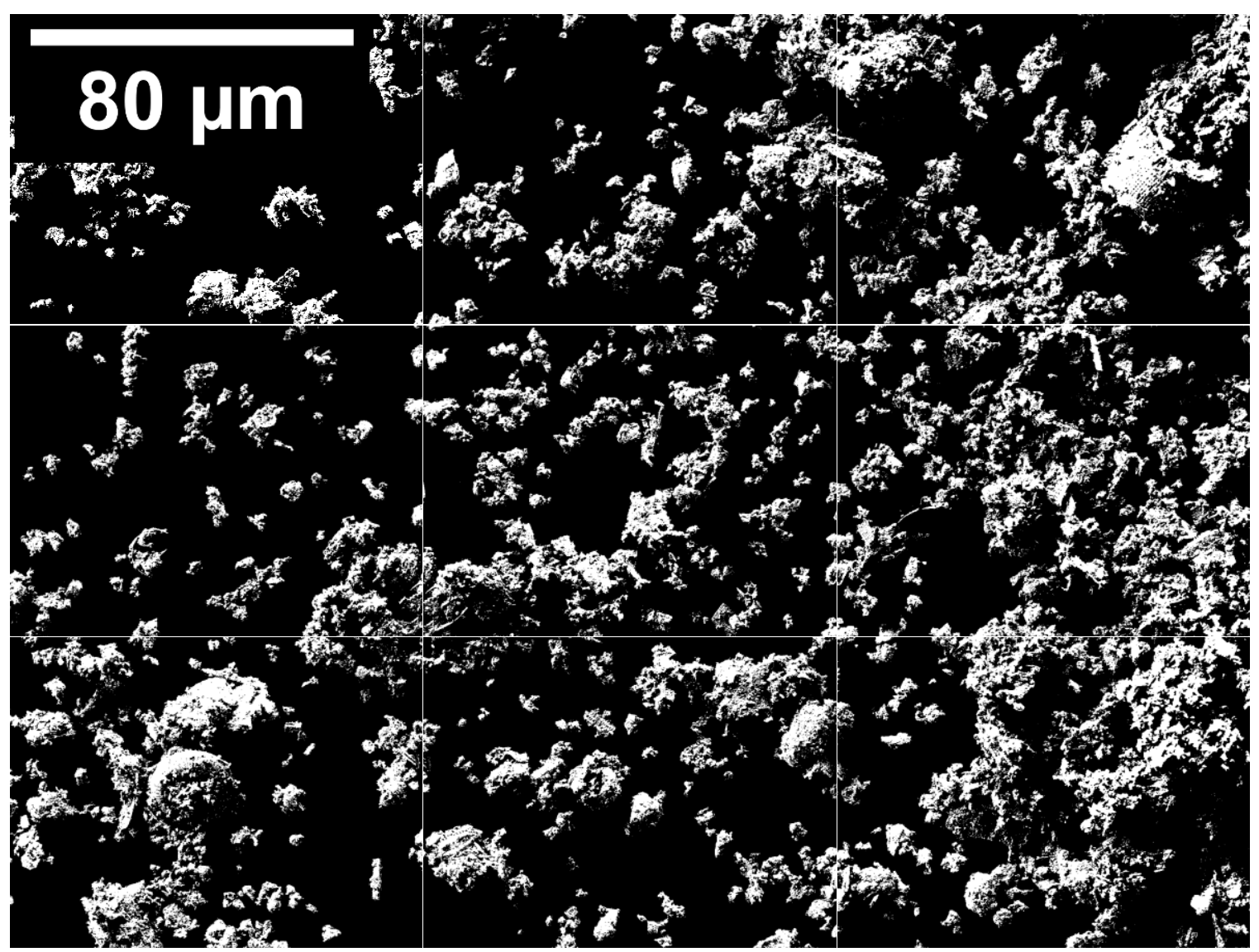

Figure 5-60. Mosaic III, $3 \times 3$ image mosaic of processed SEM images with only diatom particles on a black background from specimen 02 from the Wickiup Junction, La Pine field site. Images were captured at a magnification of 3000, and the scale bar of $80 \mu \mathrm{m}$ on the top right image applies for all images. 


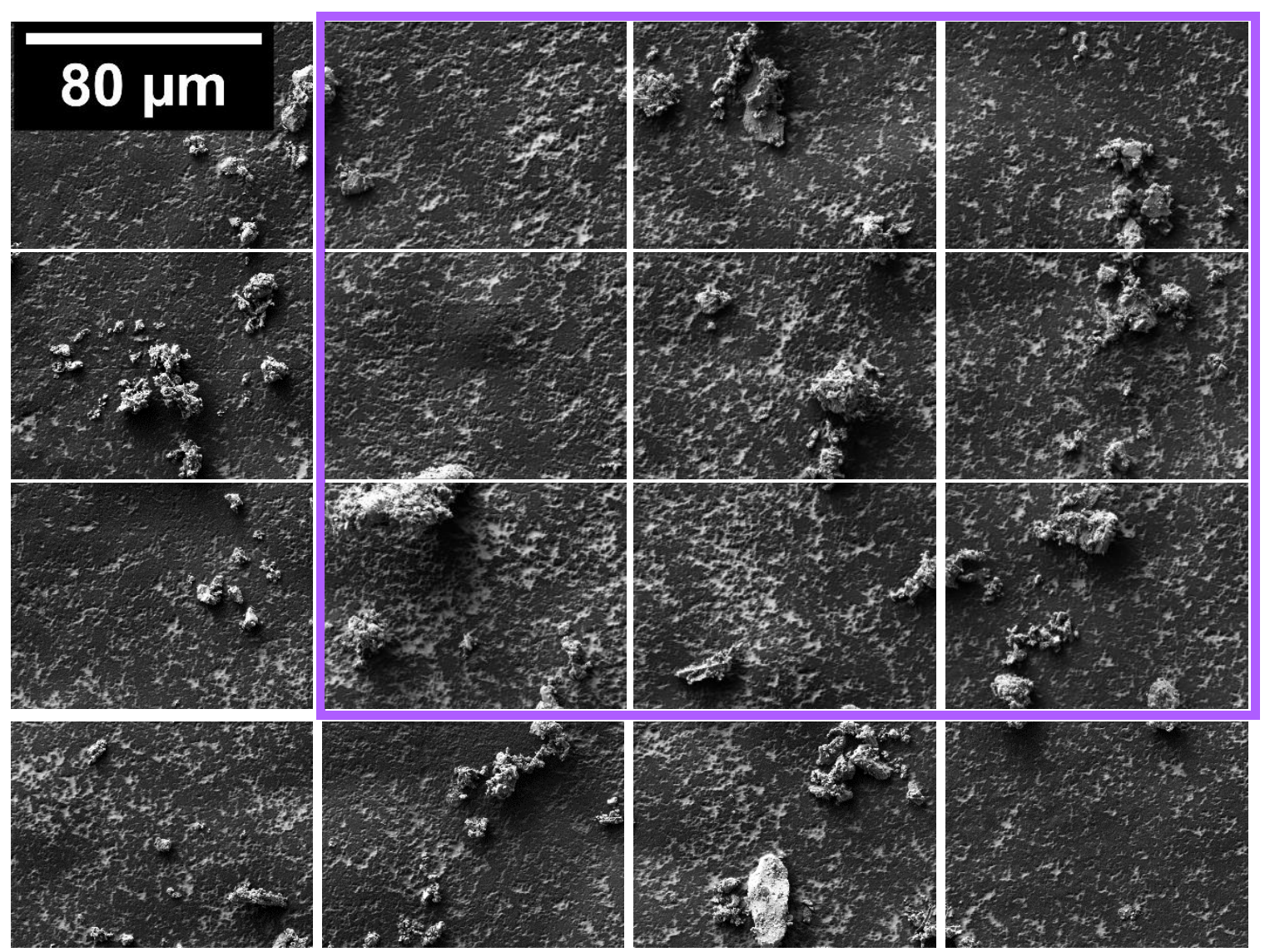

Figure 5-61. 4x4 image mosaic of original SEM images from specimen 02 from sample W1N10 from the Wickiup Junction, La Pine field site. The purple box illustrates the chosen $3 \times 3$ image mosaic chosen for analysis, Mosaic IV. Images were captured at a magnification of 3000, and the scale bar of $80 \mu \mathrm{m}$ on the top right image applies for all images. 


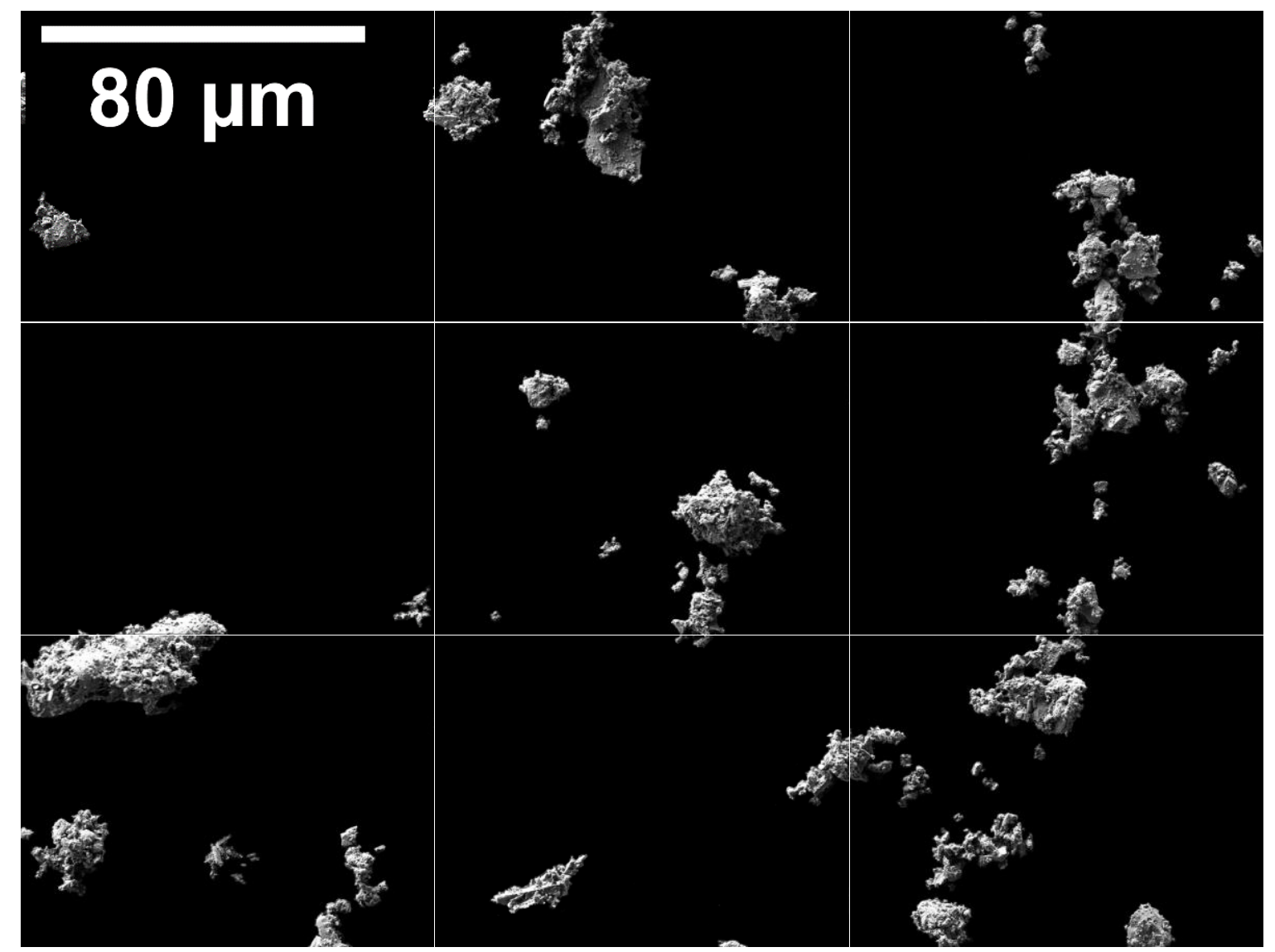

Figure 5-62. Mosaic IV, $3 \times 3$ image mosaic of SEM images with all particles on a black background from specimen 02 from the Wickiup Junction, La Pine field site. Images were captured at a magnification of 3000 , and the scale bar of $80 \mu \mathrm{m}$ on the top right image applies for all images. 


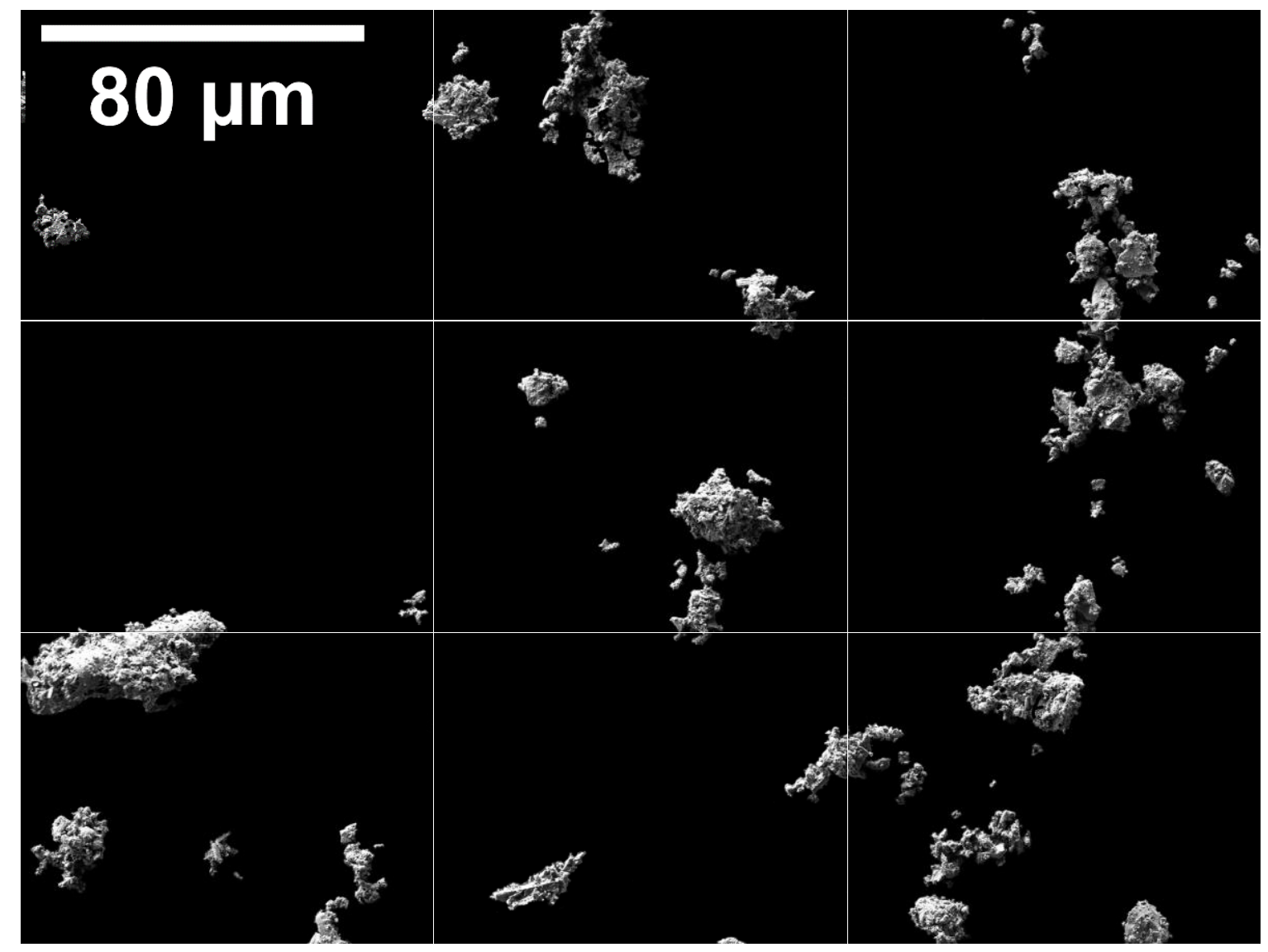

Figure 5-63. Mosaic IV, $3 \times 3$ image mosaic of SEM images with only diatom particles on a black background from specimen 02 from the Wickiup Junction, La Pine field site. Images were captured at a magnification of 3000 , and the scale bar of $80 \mu \mathrm{m}$ on the top right image applies for all images. 


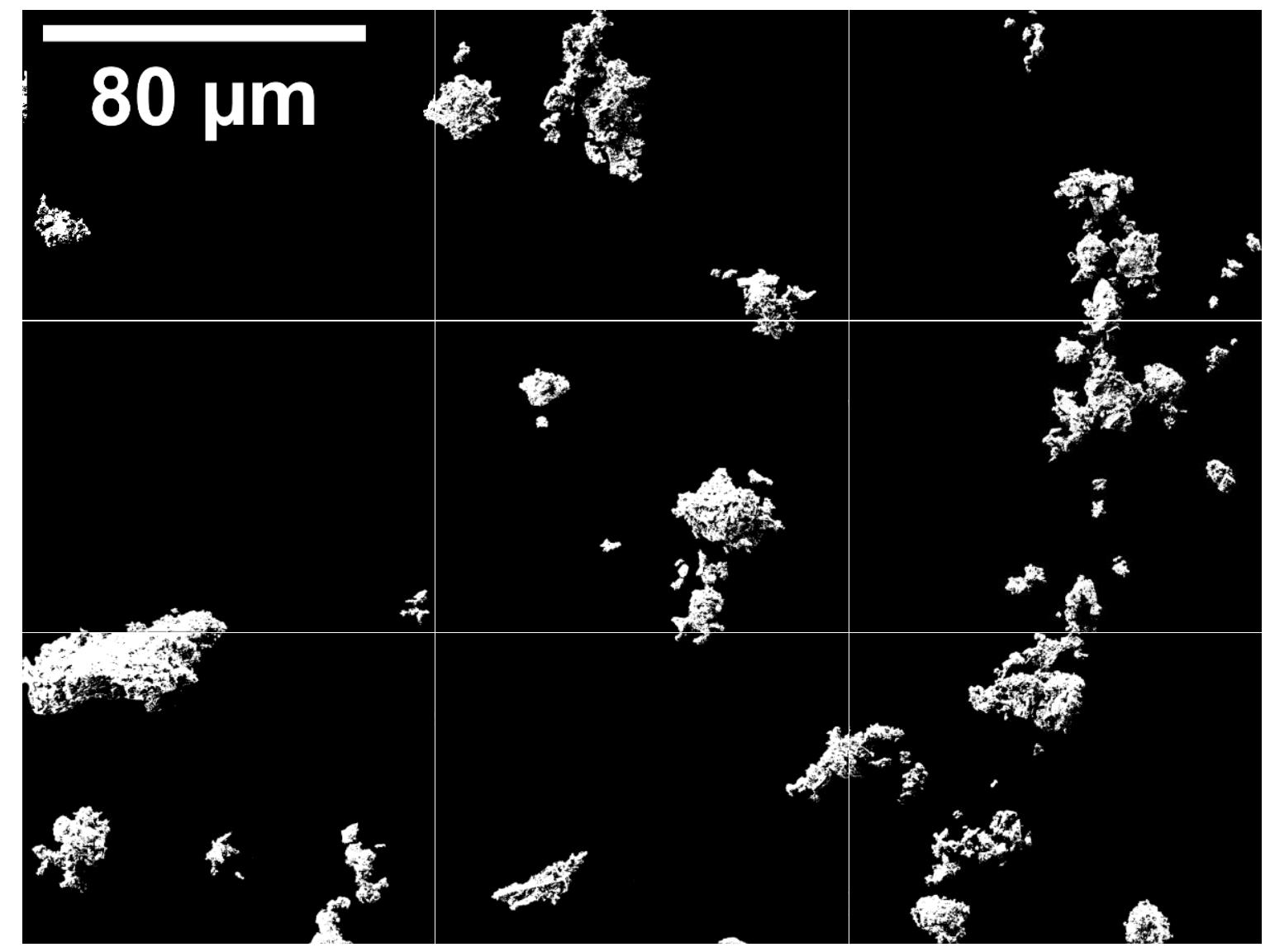

Figure 5-64. Mosaic IV, $3 \times 3$ image mosaic of processed SEM images with only diatom particles on a black background from specimen 02 from the Wickiup Junction, La Pine field site. Images were captured at a magnification of 3000, and the scale bar of $80 \mu \mathrm{m}$ on the top right image applies for all images. 


\section{APPENDIX B: NUMBER OF MOSAIC IMAGES}




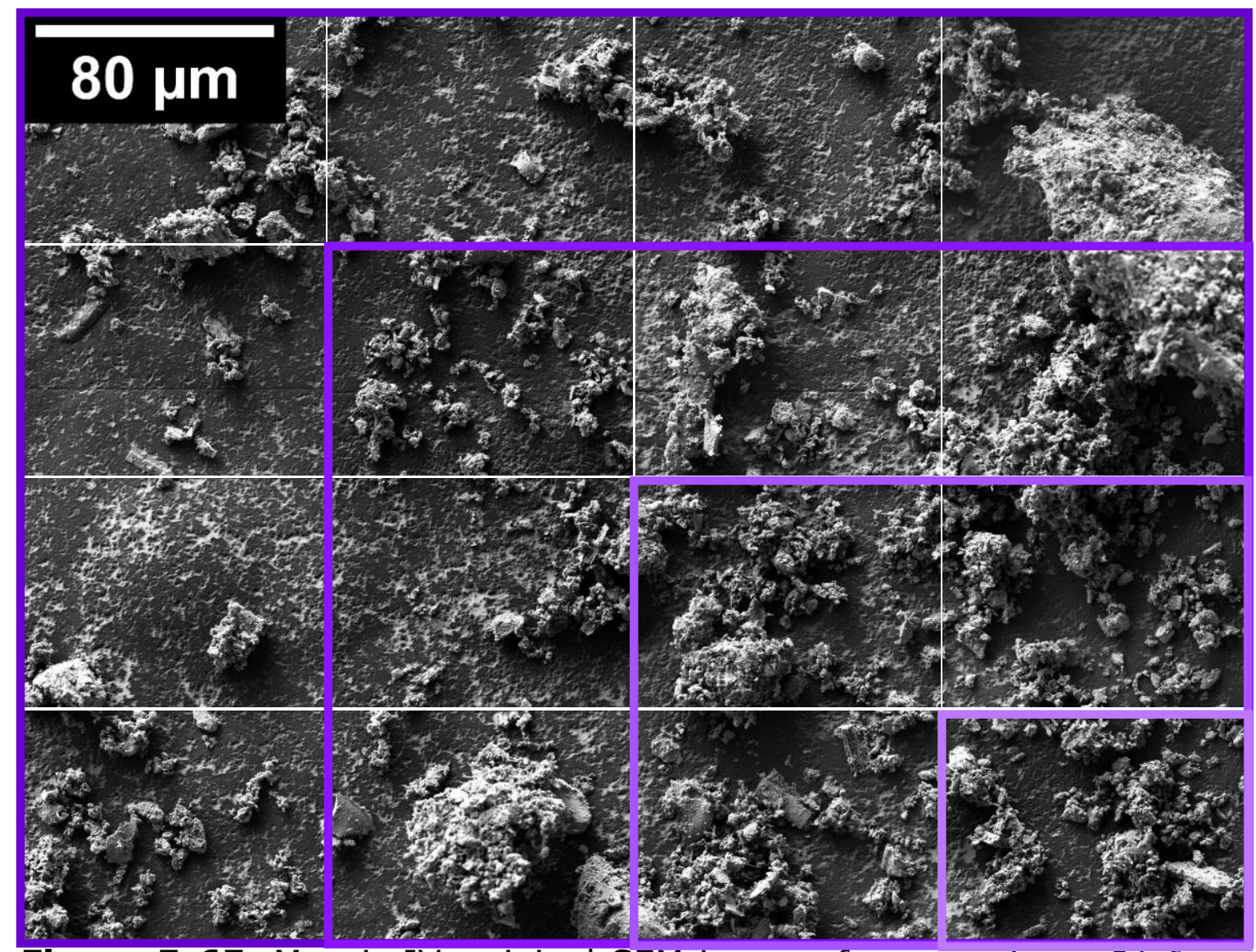

Figure 5-65. Mosaic IV, original SEM images from specimen 01 from sample W1N10 from the Wickiup Junction, La Pine field site. Boxes isolate the $4 \times 43 \times 3,2 \times 2$, and $1 \times 1$ image mosaics. Images were captured at a magnification of 3000 , and the scale bar represents a length of $80 \mu \mathrm{m}$ on the top right image applies for all images. 


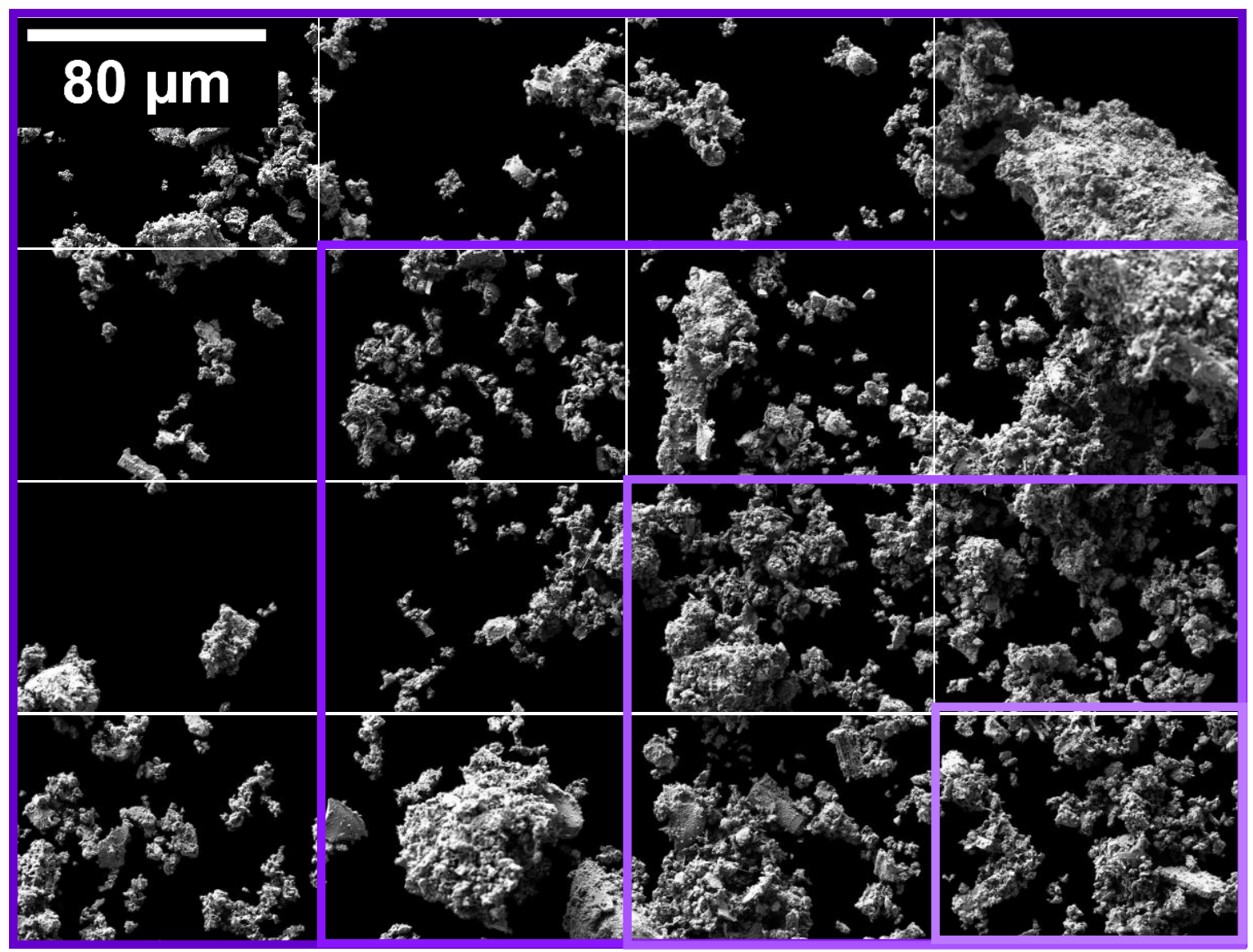

Figure 5-66. Mosaic IV, SEM images with all particles on a black background from specimen 01 from sample W1N10 from the Wickiup Junction, La Pine field site. Boxes isolate the $4 \times 43 \times 3,2 \times 2$, and $1 \times 1$ image mosaics. Images were captured at a magnification of 3000 , and the scale bar represents a length of $80 \mu \mathrm{m}$ on the top right image applies for all images. 


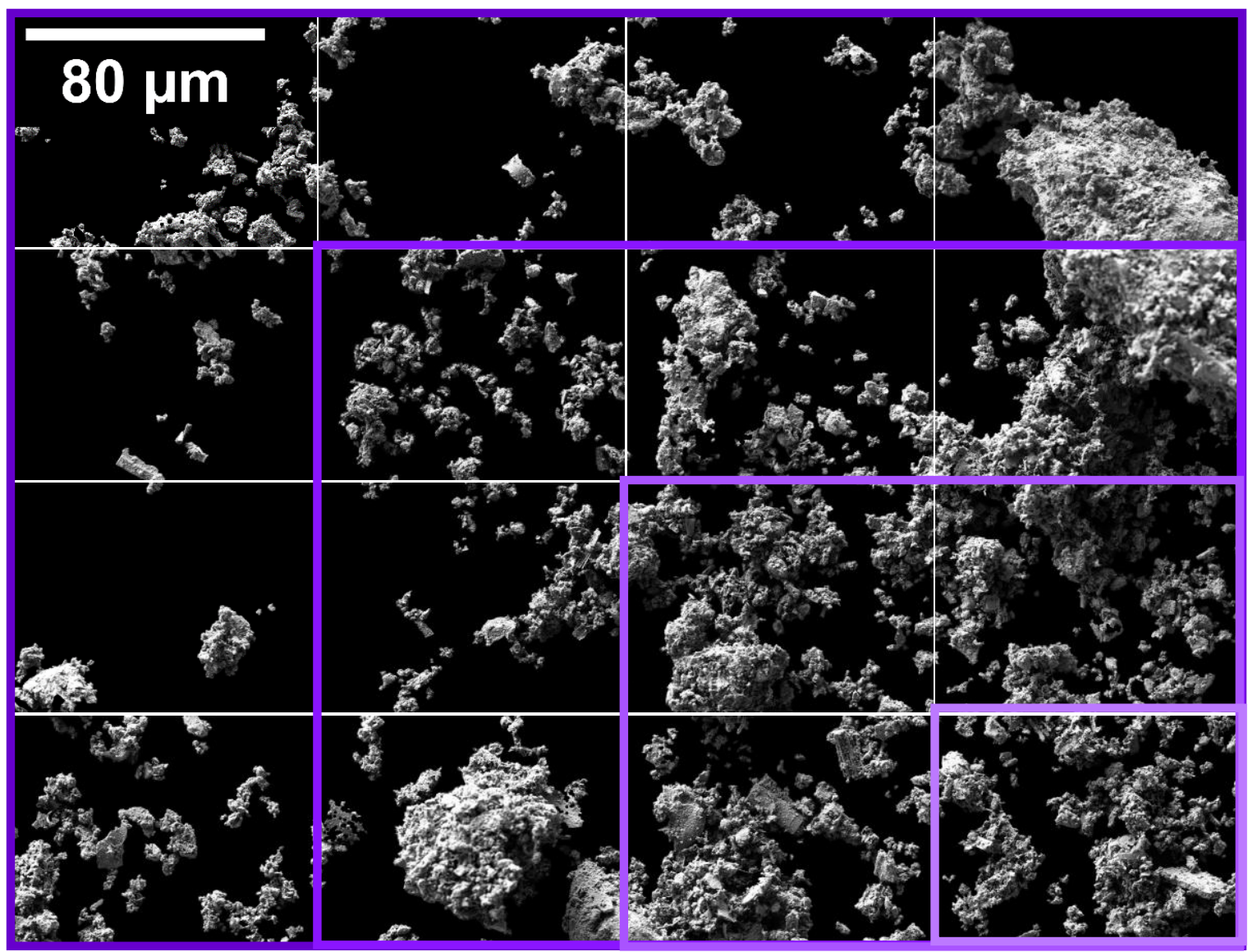

Figure 5-67. Mosaic IV, SEM images with only diatom particles on a black background from specimen 01 from sample W1N10 from the Wickiup Junction, La Pine field site. Boxes isolate the $4 \times 43 \times 3,2 \times 2$, and $1 \times 1$ image mosaics. Images were captured at a magnification of 3000 , and the scale bar represents a length of $80 \mu \mathrm{m}$ on the top right image applies for all images. 


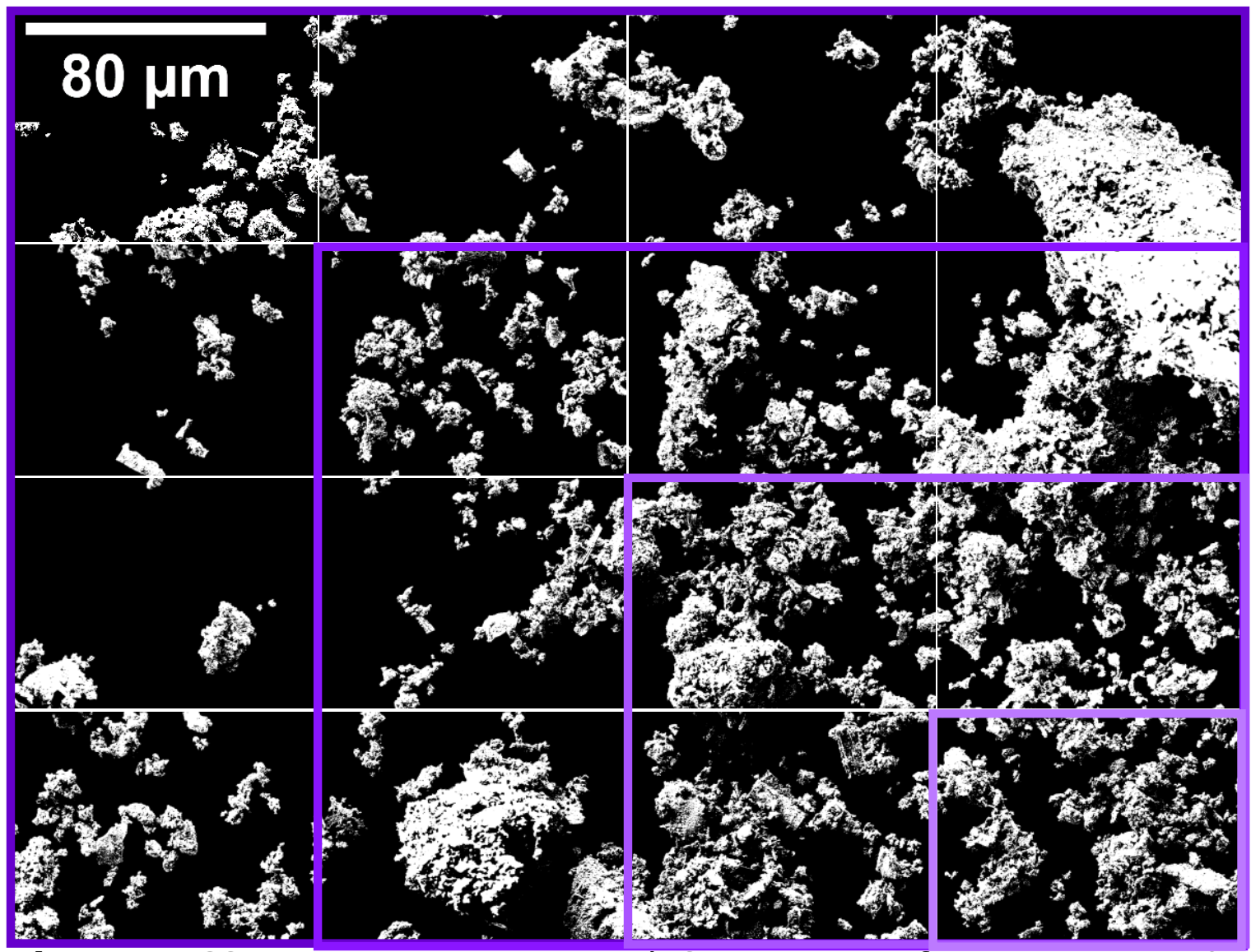

Figure 5-68. Mosaic IV, processed SEM images from specimen 01 from sample W1N10 from the Wickiup Junction, La Pine field site. Boxes isolate the $4 \times 43 \times 3,2 \times 2$, and $1 \times 1$ image mosaics. Images were captured at a magnification of 3000 , and the scale bar represents a length of $80 \mu \mathrm{m}$ on the top right image applies for all images. 


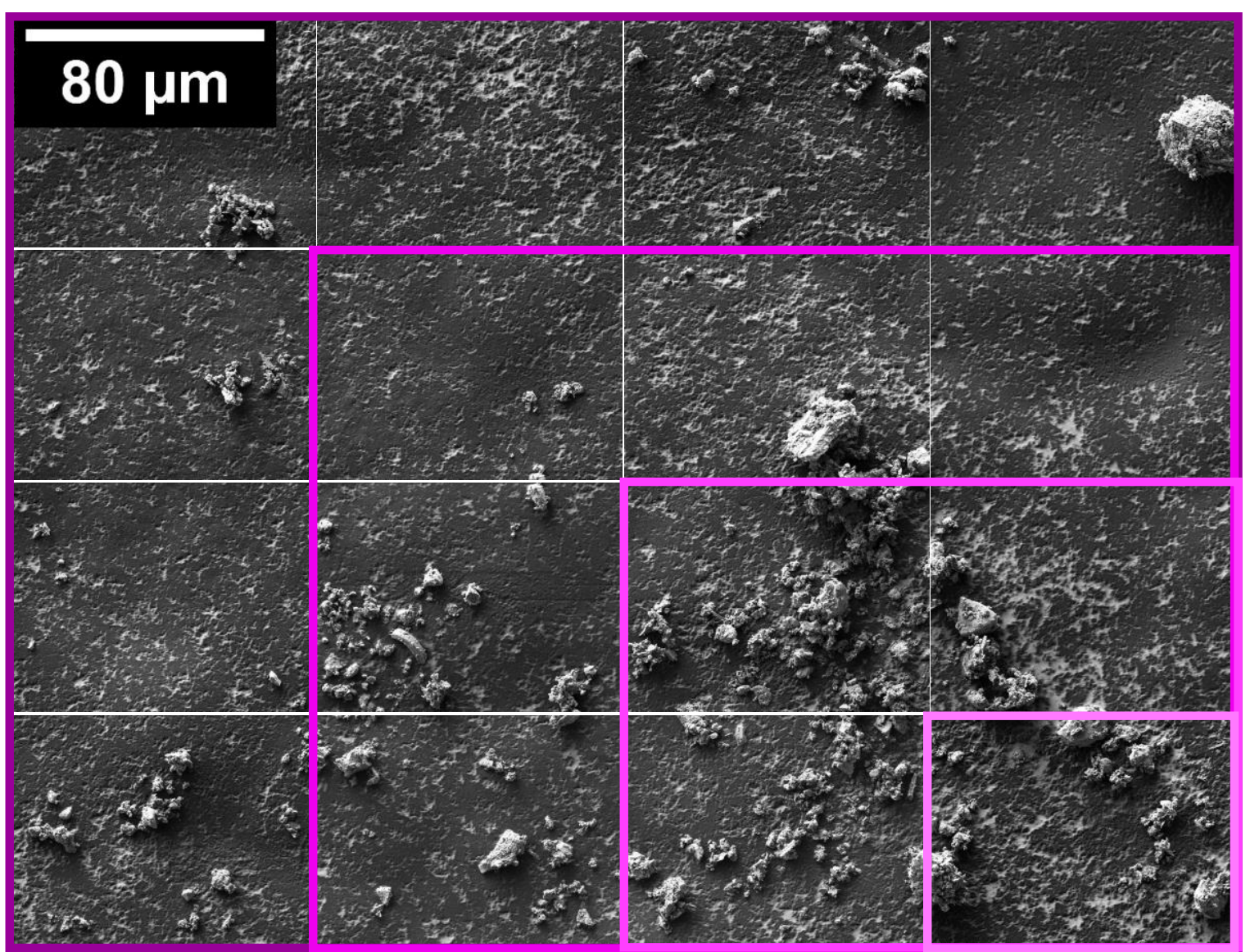

Figure 5-69. Mosaic II, original SEM images from specimen 02 from sample W1N10 from the Wickiup Junction, La Pine field site. Boxes isolate the $4 \times 43 \times 3,2 \times 2$, and $1 \times 1$ image mosaics. Images were captured at a magnification of 3000 , and the scale bar represents a length of $80 \mu \mathrm{m}$ on the top right image applies for all images. 


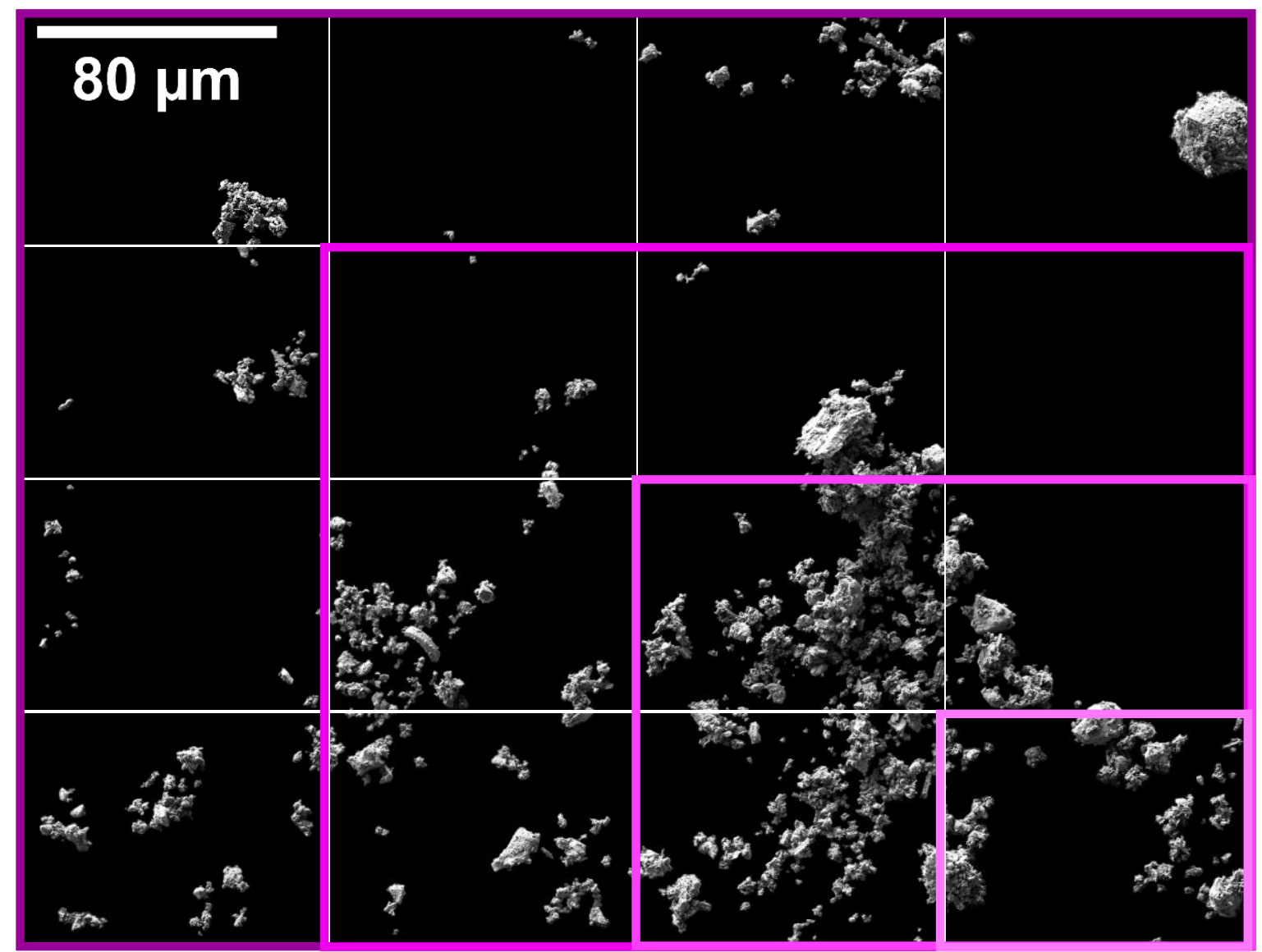

Figure 5-70. Mosaic II, SEM images with all particles on a black background from specimen 02 from sample W1N10 from the Wickiup Junction, La Pine field site. Boxes isolate the $4 \times 43 \times 3,2 \times 2$, and $1 \times 1$ image mosaics. Images were captured at a magnification of 3000 , and the scale bar represents a length of $80 \mu \mathrm{m}$ on the top right image applies for all images. 


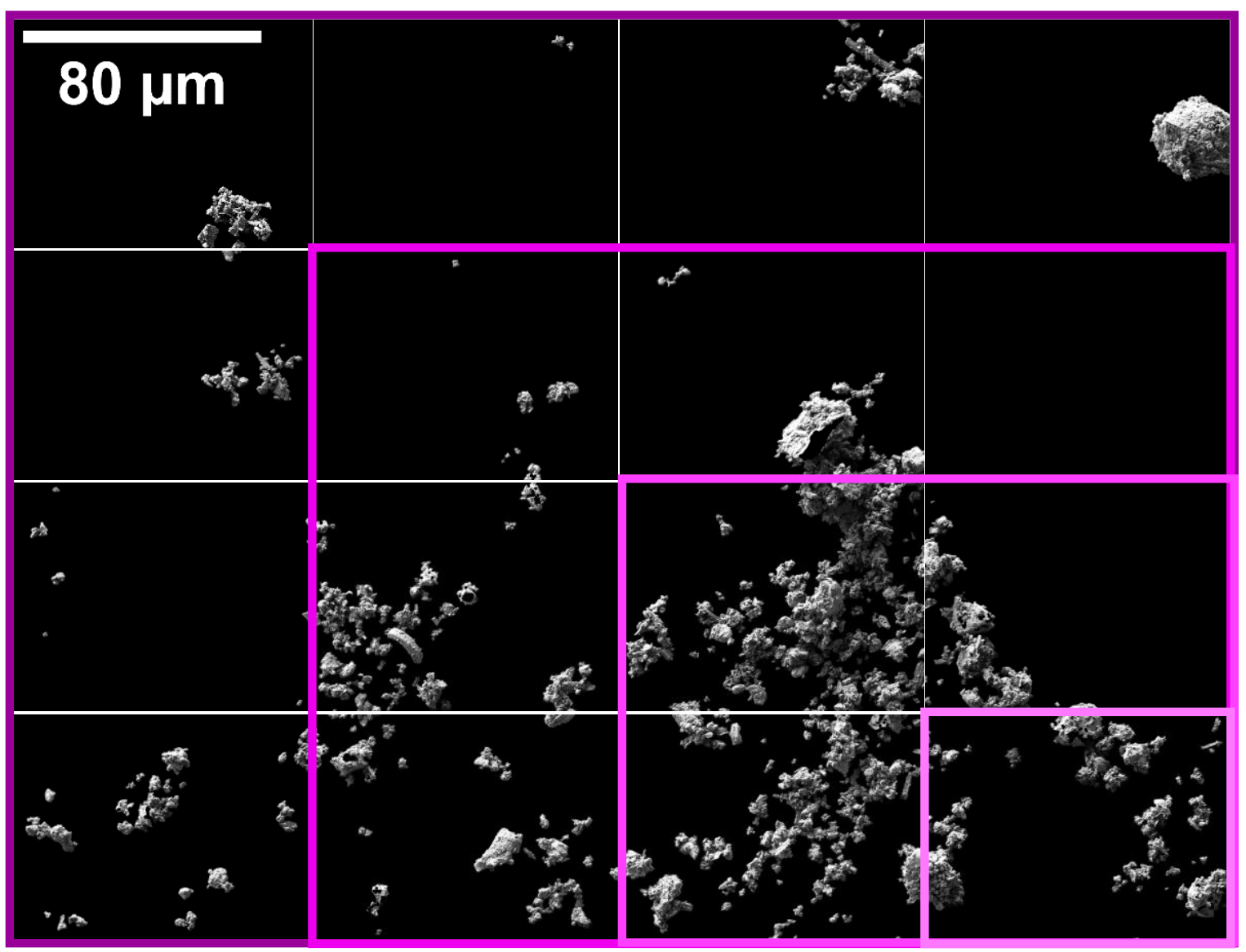

Figure 5-71. Mosaic II, SEM images with only diatom particles on a black background from specimen 02 from sample W1N10 from the Wickiup Junction, La Pine field site. Boxes isolate the $4 \times 43 \times 3,2 \times 2$, and $1 \times 1$ image mosaics. Images were captured at a magnification of 3000 , and the scale bar represents a length of $80 \mu \mathrm{m}$ on the top right image applies for all images. 


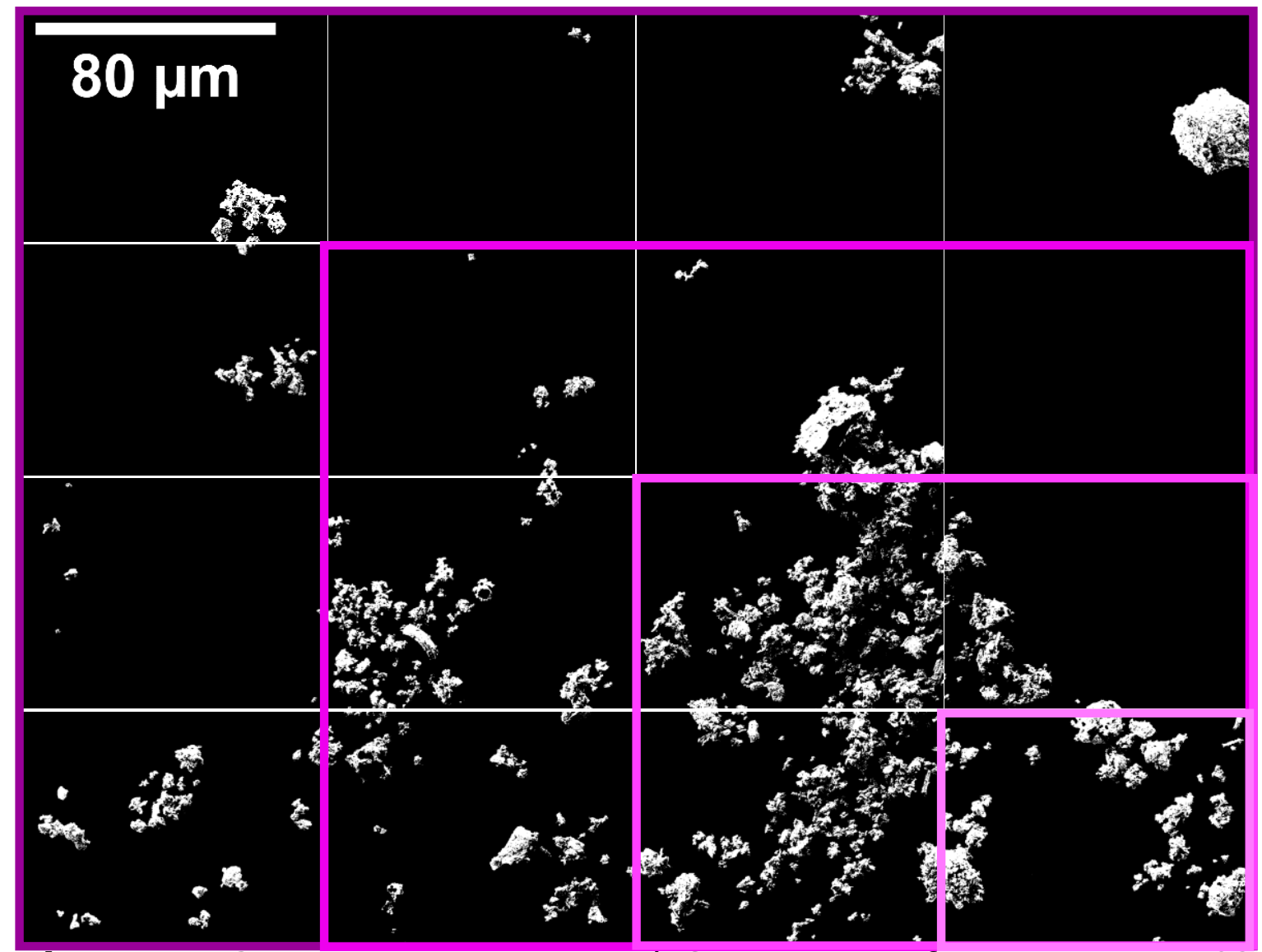

Figure 5-72. Mosaic II, processed SEM images from specimen 02 from sample W1N10 from the Wickiup Junction, La Pine field site. Boxes isolate the $4 \times 43 \times 3,2 \times 2$, and $1 \times 1$ image mosaics. Images were captured at a magnification of 3000 , and the scale bar represents a length of $80 \mu \mathrm{m}$ on the top right image applies for all images. 


\section{APPENDIX C: MATLAB CODE}


$\%$ Working directories \%

$\% \%$ The script begins by setting up links to the files containing the images to be processed. These images include: the original SEM plain images designated "-00"; the cleaned images with all particles present on a black background designated "-01"; the cleaned images with only the diatom particles on a black background designated "-02"; and the binarized images for both "-01" and "-02" images where the threshold value is added to the end of the name as "- $0 x$ ". The working directories shown are the ones the present researcher used throughout this procedure. $\% \%$

filein $1=' \backslash \backslash$ thoth. cecs. $p d x$. edu $\backslash$ Home04 $\backslash$ ariadna $\backslash$ Desktop $\backslash$ ODOT_Diatom s $\backslash$ Images $\backslash$; ;

$\% \%$ filein 1 : specifies the location of the set of cleaned images i.e., images with all particles on a black background, and with only diatom particles on a black background - in bmp format. $\% \%$

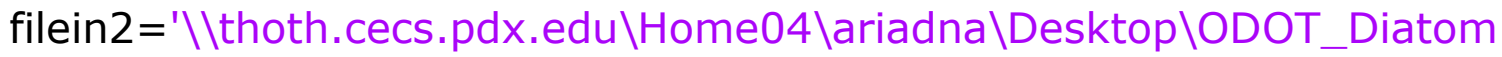
s\Outputs \';

$\% \%$ filein2: specifies the location of the binarized images images in black and white, where white represents the particles of interest - after the threshold values have been applied to the images. $\% \%$

fileout $=\backslash \backslash$ thoth.cecs.pdx.edu $\backslash$ Home04 $\backslash$ ariadna \Desktop $\backslash$ ODOT_Diatom s\Outputs \';

$\% \%$ fileout: specifies the file where the images will be saved after the threshold values have been applied to each of them, i.e., the processed images. $\% \%$

intype='.bmp';

$\% \%$ Format of the image being analyzed. \%\%

outtype $1=$ '.bmp';

$\% \%$ Format of the analyzed (or processed) image to be saved. $\% \%$

\% Image name: W1 N10-01-020 \% ACO \%

$\%$ Threshold \%

specimen $00=([$ filein 1, 'W1 N10-01-020-00'] $)$;

$\% \%$ Identifying the image of interest by name. In this case the original SEM image. \%\%

$\mathrm{M00}=$ imread $([$ specimen00, intype $])$;

$\% \%$ Instructing Matlab to read the image and its format. In this case the original SEM image. $\% \%$ 
imhist(M00)

$\% \%$ Getting the grayscale histogram of the image of interest to investigate threshold values of interest. The original SEM image is only needed to see the histogram of the image. $\% \%$

specimen01 = ([filein1, 'W1N10-01-020-01'] $)$;

$\% \%$ Identifying the image of interest by name. In this case the image cleaned to have all the particles in a black background. $\% \%$

M01 $=\operatorname{imread}([$ specimen01,intype $])$;

$\% \%$ Instructing Matlab to read the image and its format. In this case the image cleaned to have all the particles in a black background. \%\%

specimen02 $=\left(\left[\right.\right.$ filein $\left.1,{ }^{\prime} \mathbf{W} 1 \mathbf{N 1 0 - 0 1 - 0 2 0 - 0 2 ' ]}\right)$;

$\% \%$ Identifying the image of interest by name. In this case the image cleaned to only have diatom particles in a black background. \%\%

M02 $=\operatorname{imread}([$ specimen02, intype $])$;

$\% \%$ Instructing Matlab to read the image and its format. In this case the image cleaned to only have diatom particles in a black background. $\% \%$

M01_thresh $=[0.3137$ ]';

$\% \%$ Indicating the threshold value in decimal, i.e., $x / 255$, to apply to an image to binarize. Multiple threshold values can be listed within the square brackets separated by a space. \%\%

M01_out_thresh $=\{$ '-03137' $\}$;

$\% \%$ Indicates the threshold value will be added to the end of the image name. The number of threshold values listed in the previous line (M01_thresh) must match the number of values listed in this line (M01_out_thresh). The values need to be listed in the format: '- $x$ ' and separated by a space. $\% \%$

for $\mathrm{k}=1$ : length(M01_out_thresh)

$B=$ imbinarize (M01, M01_thresh(k));

imwrite(B,[fileout, 'W1N10-01-020-

01',char(M01_out_thresh(k)),outtype1]);

['File number ' num2str(k) ' has been processed'] end

$\% \%$ The loop starting from "for" and ending at "end" the indicates for Matlab to apply the threshold values to the image of interest, i.e., W1N10-01-020-01, to convert the image to binary and save it in bmp format in the file designated as "fileout". $\% \%$

M02_thresh $=[0.3137]^{\prime}$;

$\% \%$ Indicating the threshold value in decimal, i.e., $x / 255$, to apply to an image to binarize. Multiple threshold values can be 
listed within the square brackets separated by a space. \% The values listed here need to match those listed in M01_thresh. \%

M02_out_thresh $=\{$ '-03137' $\}$;

$\% \%$ Indicates the threshold value will be added to the end of the image name. The number of threshold values listed in the previous line (M02_thresh) have to match the number of values listed in this line (M02_out_thresh). The values need to be listed in the format: '- $x$ ' and separated by a space. \%

for $\mathrm{k}=1$ : length(M02_out_thresh)

$\mathrm{B}=$ imbinarize $(\mathrm{M} 02, \mathrm{M} 02$ _thresh $(\mathrm{k}))$;

imwrite(B,[fileout, 'W1N10-01-020-02',

char(M02_out_thresh(k)),outtype1]);

['File number ' num2str(k) ' has been processed'] end

$\% \%$ The loop starting from "for" and ending at "end" the indicates for Matlab to apply the threshold values to the image of interest, i.e., W1N10-01-020-02, to convert the image to binary and save it in bmp format in the file designated as "fileout". \%\%

$\%$ void ratio $(V R, e) \%$

M01 =imread([filein2,'WJ N10-01-020-01-03137.bmp']);

$\% \%$ Instructing Matlab to read the binarized image of interest located in filein $2 . \% \%$

M02=imread([filein2,'WJ N10-01-020-02-03137.bmp']);

$\% \%$ Instructing Matlab to read the binarized image of interest located in filein2. \%\%

$[r, c]=\operatorname{size}(M 01)$;

$\% \%$ Pixel size of the image W1N10-01-020-01. \%\%

vs $=\operatorname{sum}(\operatorname{sum}(\mathrm{M} 01))$;

$\% \%$ vs refers to the volume of solids, which in this case includes the volume of diatom and non-diatom particles. $\% \%$ $\mathrm{vt}=\mathrm{r}^{*} \mathrm{c}$;

$\% \%$ vt refers to the total volume within the image calculated by multiplying ( $r$ ) by (c). \%\%

$\mathrm{vv}=\mathrm{vt}-\mathrm{vs}$;

$\% \% \mathrm{vv}$ refers to the volume of void within the image of interest calculated as the difference between the total volume and the volume of solids. $\% \%$

$\mathrm{e} 01=\mathrm{vv} / \mathrm{vs}$;

$\% \%$ e01 refers to the void ratio of image W1N10-01-020-0103137.bmp with all particles present on a black background this is calculated by dividing the volume of void by the volume of solids. e01 $=e_{\text {all particles }} \% \%$ 


$$
[\mathrm{r}, \mathrm{c}]=\operatorname{size}(\mathrm{M} 02)
$$

$\% \%$ Pixel size of the image W1N10-01-020-02. \%\% vs $=\operatorname{sum}(\operatorname{sum}(\mathrm{M} 02))$;

$\% \%$ vs refers to the volume of solids, which in this case includes the volume of diatom and non-diatom particles. $\% \%$ $\mathrm{vt}=\mathrm{r}^{*} \mathrm{c} ;$ $\% \%$ vt refers to the total volume within the image calculated by multiplying ( $r$ ) by (c). \%\%

$\mathrm{vv}=\mathrm{vt}-\mathrm{vs}$;

$\% \% \mathrm{vv}$ refers to the volume of void within the image of interest calculated as the difference between the total volume and the volume of solids. $\% \%$

$\mathrm{e} 02=\mathrm{vv} / \mathrm{vs}$;

$\% \%$ e02 refers to the void ratio of image W1N10-01-020-0103137.bmp with all particles present on a black background this is calculated by dividing the volume of void by the volume of solids. e02 $=e_{\text {diatom }} \% \%$ 